JOURNAL OF THE

AMERICAN MATHEMATICAL SOCIETY

Volume 24, Number 3, July 2011, Pages 709-769

S 0894-0347(2011)00693-0

Article electronically published on January 25, 2011

\title{
RATIONAL POINTS OF UNIVERSAL CURVES
}

\author{
RICHARD HAIN
}

\section{CONTEnTs}

1. Introduction

2. Fundamental groups and Galois groups 713

3. Points and sections $\quad 714$

4. Monodromy representations $\quad 715$

5. Mapping class groups and moduli stacks of curves 717

6. Relative and weighted completion of profinite groups $\quad 719$

7. Weighted completion and families of curves $\quad 725$

8. Weighted completion of arithmetic mapping class groups 728

9. Generators and relations $\quad 733$

10. The Lie algebra $\mathfrak{d}_{g, n} \quad 741$

11. Topologically ample families of curves 745

12. The class of a rational point 748

13. Computation of $C(k(T)) \quad 750$

14. Non-abelian cohomology 751

15. Setup for proofs of Theorems 2 and 3

16. Cohomology computations 757

17. Computation of $H^{\bullet}\left(\mathfrak{g}_{K}, \mathrm{Gr}_{\bullet}^{W} \mathfrak{p}\right) \quad 760$

18. Proofs of Theorems 2 and 3

Appendix. A Hodge theoretic lemma $\quad 765$

Acknowledgments $\quad 767$

References

\section{INTRODUCTION}

Associated to a smooth, projective, geometrically connected curve $C$ over a field $K$ is the short exact sequence

$$
1 \rightarrow \pi_{1}\left(C \otimes_{K} \bar{K}, \bar{\eta}\right) \rightarrow \pi_{1}(C, \bar{\eta}) \rightarrow G_{K} \rightarrow 1
$$

of étale fundamental groups, where $\bar{K}$ is a separable closure of $K, G_{K}=\operatorname{Gal}(\bar{K} / K)$ and where $\bar{\eta}$ is a geometric point of $C$. Each $K$-rational point $x$ of $C$ induces a

Received by the editors January 27, 2010 and, in revised form, September 19, 2010, and December 29, 2010.

2010 Mathematics Subject Classification. Primary 14G05, 14G27, 14H10, 14H25; Secondary 11G30, 14G32.

The author was supported in part by grant DMS-0706955 from the National Science Foundation and by MSRI.

(C)2011 American Mathematical Society Reverts to public domain 28 years from publication 
section $s_{x}: G_{K} \rightarrow \pi_{1}(C, \bar{\eta})$ of the right-hand map, which is well defined up to conjugation by an element of the geometric fundamental group $\pi_{1}\left(C \otimes_{K} \bar{K}, \bar{\eta}\right)$. Grothendieck's Section Conjecture [12] asserts that, if $K$ is an infinite field that is finitely generated over its prime field, and if the genus of $C$ is 2 or more, then the function

$$
C(K) \rightarrow\left\{\text { sections of } \pi_{1}(C, \bar{\eta}) \rightarrow G_{K}\right\} / \text { conjugation by } \pi_{1}\left(C \otimes_{K} \bar{K}, \bar{\eta}\right)
$$

that takes $x$ to the conjugacy class of $s_{x}$ is a bijection. In this paper we prove the section conjecture for the restriction of the universal curve $\mathcal{C} \rightarrow \mathcal{M}_{g}$ to its generic point $\operatorname{Spec}\left(k\left(\mathcal{M}_{g}\right)\right)$ for all $g \geq 5$ when, for example, $k$ is a number field 1 When $g \geq 5$ and $n \geq 1$ we prove a modified version of the Section Conjecture for the pullback of the universal curve to the generic point of $\mathcal{M}_{g, n}$ in which $\pi_{1}\left(C \otimes_{K} \bar{K}, \bar{\eta}\right)$ is replaced by its $\ell$-adic prounipotent completion, where the prime number $\ell$ is chosen so that the image of the $\ell$-adic cyclotomic character $\chi_{\ell}: G_{k} \rightarrow \mathbb{Z}_{\ell}^{\times}$is infinite. In particular, this version of the "unipotent section conjecture" holds whenever $k$ is a number field or a finite extension of $\mathbb{Q}_{p}$, where $p \neq \ell$.

The proof proceeds in two stages. We first show that, when $g \geq 3$, the universal curve over $\mathcal{M}_{g, n}$ has no more $k\left(\mathcal{M}_{g, n}\right)$-rational points other than the obvious ones, namely, the rational points that are the restrictions of the $n$ tautological sections of $\mathcal{C} \rightarrow \mathcal{M}_{g, n}$. This follows directly from results in Teichmüller theory proved by Hubbard [25] and Earle and Kra 6] in the 1970s. We give an algebraic proof of this consequence of their results which also applies to ample subvarieties of $\mathcal{M}_{g, n}$ of dimension $\geq 2$ and which should also hold in positive characteristic.

The second step is to study conjugacy classes of sections of the group extension

$$
1 \rightarrow \pi_{1}\left(C \otimes_{K} \bar{K}, \bar{\eta}\right)_{/ \mathbb{Q}_{\ell}}^{\text {un }} \rightarrow G_{K} \ltimes \pi_{1}\left(C \otimes_{K} \bar{K}, \bar{\eta}\right)_{/ \mathbb{Q}_{\ell}}^{\text {un }} \rightarrow G_{K} \rightarrow 1,
$$

where $K=k\left(\mathcal{M}_{g, n}\right), n \geq 1$, and where $C$ is the pullback of the universal curve to Spec $K$. This is done using the non-abelian cohomology developed by Kim in 28 for extensions of a profinite group by a prounipotent group, which proves to be very effective in this case.

The principal tool used to prove these results is the theory of weighted completions of profinite groups [20, 23, which was developed with Makoto Matsumoto. Other notable ingredients used include the computation of the relative completion of mapping class groups in genus $\geq 3$ in [15, a "density theorem" [18], and Kim's non-abelian cohomology mentioned above.

This work is inspired by work of Ellenberg [7] and Kim [29] who independently tried to bound the number of rational points of hyperbolic curves over a number field by bounding the number of conjugacy classes of sections of the surjective homomorphism $\pi_{1}(C, \bar{\eta}) / L^{r} \rightarrow G_{K}$, where $L^{r}$ denotes the $r$ th term of the lower central series of $\pi_{1}\left(C \otimes_{K} \bar{K}, \bar{\eta}\right)$.

We now describe the main results in more detail. A natural generalization of the universal curve $\mathcal{C} \rightarrow \mathcal{M}_{g, n}$, introduced in Section 11, is that of a topologically ample family of curves of type $(g, n)$. Roughly speaking, a family $C \rightarrow T$ of smooth projective curves over a geometrically connected $k$-variety $T$ is topologically ample of type $(g, n)$, where $g \geq 3$, if it has $n$ disjoint sections and if the corresponding $k$ morphism $T \rightarrow \mathcal{M}_{g, n}$ induces an isomorphism of the fundamental group of $T$ with a finite index subgroup of $\pi_{1}\left(\mathcal{M}_{g, n}, \bar{\eta}\right)$ that contains the profinite Torelli group.

\footnotetext{
${ }^{1}$ Here and throughout the introduction, $\mathcal{M}_{g}, \mathcal{M}_{g, n}$ and $\mathcal{M}_{g, n}[m]$ are considered to be stacks over the field $k$.
} 
(See Definition 11.1]) Examples of topologically ample curves of type $(g, n)$ include the universal curve over the moduli space $\mathcal{M}_{g, n}[m]$ of smooth projective curves of type $(g, n)$ with a level $m$ structure, where $g+n \geq 4$ or $g \geq 3$ and $m \geq 3$, and its restriction to a generic linear section of $\mathcal{M}_{g, n}[\mathrm{~m}]$ of dimension $\geq 2$.

Theorem 1. Suppose that $C \rightarrow T$ is a topologically ample family of curves of type $(g, n)$ over a field $k$ of characteristic zero. If $g \geq 3$, then the only $k(T)$-rational points of $C$ are its $n$ tautological points: $C(k(T))=\left\{x_{1}, \ldots, x_{n}\right\}$.

The theorem is false when $g=2$. Since every genus 2 curve is hyperelliptic,

$$
\left|C\left(k\left(\mathcal{M}_{2, n}[2 m]\right)\right)\right| \geq 6+2 n
$$

whenever $m+n \geq 2$ as, apart from the $n$ tautological sections, $C\left(k\left(\mathcal{M}_{2, n}[2 m]\right)\right)$ contains the $2 g+2=6$ Weierstrass points of the universal curve and the $n$ hyperelliptic conjugates of the tautological points 2 In fact, the result of Earle-Kra [6] implies that there are no additional rational points, so that one has equality above.

The following result establishes the Section Conjecture for the pullback of the universal curve to the generic point of $\mathcal{M}_{g}[m]$ when $g \geq 5$, provided that there exists a prime number $\ell$ such that the image of the $\ell$-adic cyclotomic character $\chi_{\ell}: G_{k} \rightarrow \mathbb{Z}_{\ell}^{\times}$is infinite.

Theorem 2. Suppose that $k$ is a field of characteristic zero, that $\ell$ is a prime number, and that $m \geq 1$. Set $K=k\left(\mathcal{M}_{g}[m]\right)$ and let $C / K$ be the pullback of the universal genus $g$ curve to Spec $K$. Fix a geometric point $\bar{\eta}$ of $C$. If $g \geq 5$, and if the image of the $\ell$-adic cyclotomic character $\chi_{\ell}: G_{k} \rightarrow \mathbb{Z}_{\ell}^{\times}$is infinite, then the extension

$$
1 \rightarrow \pi_{1}\left(C \otimes_{K} \bar{K}, \bar{\eta}\right) \rightarrow \pi_{1}(C, \bar{\eta}) \rightarrow G_{K} \rightarrow 1
$$

does not split.

When $n \geq 1$, we can only prove a weaker version of this result. As in the previous result, $k$ is a field of characteristic zero. Fix $m \geq 1$ and set $K=k\left(\mathcal{M}_{g, n}[m]\right)$. Let $C / K$ be the pullback of the universal curve to Spec $K$.

Each $K$-rational point $x_{j} \in\left\{x_{1}, \ldots, x_{n}\right\}$ of $C$ induces a continuous homomorphism

$$
s_{j}: G_{K} \rightarrow \text { Aut } \pi_{1}\left(C \otimes_{K} \bar{K}, \bar{x}_{j}\right),
$$

where $\bar{x}_{j}: \operatorname{Spec} \bar{K} \rightarrow C$ is a geometric point that lies over $x_{j}$. This induces a homomorphism

$$
s_{j}^{\text {un }}: G_{K} \rightarrow \text { Aut } \pi_{1}\left(C \otimes_{K} \bar{K}, \bar{x}_{j}\right)_{/ \mathbb{Q}_{\ell}}^{\text {un }}
$$

into the automorphism group of the $\mathbb{Q}_{\ell}$-unipotent completion of $\pi_{1}\left(C \otimes_{K} \bar{K}, \bar{x}_{j}\right)$.

Theorem 3. Suppose that $g \geq 5$ and that $n \geq 1$. Suppose that $k$ is a field of characteristic zero and that $\ell$ is a prime number for which the $\ell$-adic cyclotomic character $\chi_{\ell}: G_{k} \rightarrow \mathbb{Z}_{\ell}^{\times}$has infinite image. With the notation introduced above, if $H^{1}\left(G_{k}, \mathbb{Q}_{\ell}(r)\right)$ is finite dimensional for all $r>1$, then there is a natural bijection between the $\pi_{1}\left(C \otimes_{K} \bar{K}, \bar{x}_{1}\right)^{\mathrm{un}}\left(\mathbb{Q}_{\ell}\right)$ conjugacy classes of splittings of

$$
1 \rightarrow \pi_{1}\left(C \otimes_{K} \bar{K}, \bar{x}_{1}\right)^{\mathrm{un}}\left(\mathbb{Q}_{\ell}\right) \rightarrow G_{K} \ltimes_{s_{1}^{\text {un }}} \pi_{1}\left(C \otimes_{K} \bar{K}, \bar{x}_{1}\right)^{\text {un }}\left(\mathbb{Q}_{\ell}\right) \rightarrow G_{K} \rightarrow 1
$$

and elements of $C(K)$.

\footnotetext{
${ }^{2}$ It is necessary to take $m+n \geq 2$ as the stack $\mathcal{M}_{2}[2]$ does not have a function field. The Weierstrass points are rational as the level is even, which implies that the 2-torsion of the Jacobian is trivialized.
} 
The theorem applies, for example, when $k$ is a number field and when $k$ is a finite extension of $\mathbb{Q}_{p}$ provided that $\ell \neq p$. It should also hold when $k$ is a finite field of characteristic $p$ and $\ell \neq p$. Truncated versions of this result are proved in Section 18. The theorem also holds when $\mathcal{M}_{g, n / k}[m]$ is replaced by a generic section of it by a complete intersection $T$ of dimension $\geq 3$ and sufficiently high multi-degree. The details of the proof of this extension, Theorem 18.7 are similar to those of Theorem 3 and are left to the interested reader.

When $C$ is an affine curve, the story is more subtle as the second weight graded quotient of $\pi_{1}^{\text {un }}\left(C \otimes_{K} \bar{K}, \bar{\eta}\right)$ contains at least one copy of $\mathbb{Q}_{\ell}(1)$. Since $H^{1}\left(G_{K}, \mathbb{Q}_{\ell}(1)\right)$ is infinite dimensional, this implies that $H^{1}\left(G_{K}, \mathrm{Gr}_{-2}^{W} \pi_{1}^{\text {un }}\left(C \otimes_{K} \bar{K}\right)\right)$ is infinite dimensional when $C$ is affine. To avoid unnecessary technicalities, the affine case has been postponed.

As remarked above, the principal tool used to prove the main results is the theory of weighted completion of profinite groups developed with Makoto Matsumoto in 20, 23. Given a curve $C / T$ satisfying certain natural conditions, one can take the weighted completion of the groups $\pi_{1}\left(C, \bar{\eta}_{C}\right)$ and $\pi_{1}(T, \bar{\eta})$ with respect to their natural monodromy representations in $\operatorname{GSp}(H):=\operatorname{GSp}\left(H_{\text {ét }}^{1}\left(C_{\bar{\eta}}, \mathbb{Q}_{\ell}(1)\right)\right)$ to obtain $\mathbb{Q}_{\ell}$-affine proalgebraic groups $\mathcal{G}_{C}$ and $\mathcal{G}_{T}$, both of which are extensions of $\operatorname{GSp}(H)$ by a prounipotent group. The projection $C \rightarrow T$ induces a surjective homomorphism

$$
\mathcal{G}_{C} \rightarrow \mathcal{G}_{T}
$$

Sections of $\pi_{1}\left(C, \bar{\eta}_{C}\right) \rightarrow \pi_{1}(T, \bar{\eta})$ induce sections of (11). Theorem 1 is proved by exploiting the presentation of the Lie algebra $\mathfrak{u}_{g, n}$ of the pronilpotent radical of the relative completion of the mapping class group $\pi_{1}\left(\mathcal{M}_{g, n}^{\text {an }}, x\right)$ that was computed in [15.

The Lie algebras $\mathfrak{u}_{T}, \mathfrak{u}_{C}$ and $\mathfrak{u}_{g, n}$ of the prounipotent radicals of the fundamental groups of $T, C$ and $\mathcal{M}_{g, n}$ all have a natural weight filtration

$$
\mathfrak{u}=W_{-1} \mathfrak{u} \supseteq W_{-2} \mathfrak{u} \supseteq W_{-3} \mathfrak{u} \supseteq \cdots
$$

whose graded quotients are $\operatorname{GSp}(H)$-modules. To prove Theorem 1, one need only use their quotients $\mathfrak{u}_{C} / W_{-3}$ and $\mathfrak{u}_{T} / W_{-3}$ by $W_{-3}$. The principal defining property of a topologically ample family $C / T$ of curves of type $(g, n)$ is that the morphism $T \rightarrow \mathcal{M}_{g, n}$ induces an isomorphism $\mathfrak{u}_{T} / W_{-3} \rightarrow \mathfrak{u}_{g, n} / W_{-3}$. This clearly holds when $T \rightarrow \mathcal{M}_{g, n}$ induces an isomorphism on fundamental groups, such as when $T$ is a generic linear section of $\mathcal{M}_{g, n}$ of dimension $\geq 2$.

To prove Theorems 2 and 3 , one needs to understand how $\mathfrak{u}_{T} / W_{-3}$ changes when one replaces $T=\mathcal{M}_{g, n}$ by a Zariski open subset $U$. It turns out that when $g \geq 5$, the induced Lie algebra homomorphism

$$
\mathfrak{u}_{U} / W_{-3} \rightarrow \mathfrak{u}_{g, n} / W_{-3}
$$

is close to being an isomorphism. More precisely, its weight -1 quotient does not change when one replaces $\mathcal{M}_{g, n}$ by $U$, and its weight -2 quotient is an isomorphism modulo the trivial representation of $\operatorname{Sp}(H)$. This is proved using a corollary of the "density theorem" from [18, which asserts that if $X$ is a complex algebraic manifold, and $f: X \rightarrow \mathcal{M}_{g, n}^{\text {an }}$ a morphism whose image is a divisor, then the image of the natural monodromy representation $\pi_{1}\left(X, x_{o}\right) \rightarrow \mathrm{Sp}_{g}(\mathbb{Z})$ has finite index in $\operatorname{Sp}_{g}(\mathbb{Z})$. The proof of this density theorem makes essential use of Goresky and MacPherson's stratified Morse theory [10] and a recent result [38] of Putman on the Picard groups of level coverings of $\mathcal{M}_{g, n}$ for $g \geq 5$. 
This invariance of $\mathfrak{u} / W_{3}$ is encoded in the cohomological computations of Sections 16 and 17. Theorems 2 and 3 are then proved using Kim's non-abelian cohomology [28] and provide an example where his methods effectively find all of the rational points of a curve. These non-abelian computations are also related to, and might illuminate, work of Jordan Ellenberg [7] and Kirsten Wickelgren [45] on rational points of curves over number fields.

The computations and arguments in Sections 16 through 18 introduce certain cohomology classes of degree $\leq 2$ in the cohomology of $\mathcal{M}_{g, n}$ with coefficients in local systems corresponding to certain $\operatorname{GSp}(H)$-modules $V$. Since a smooth projective curve of genus $g$ over an extension field $K$ of $k$ with $n$-known rational points corresponds to a map Spec $K \rightarrow \mathcal{M}_{g, n}$, these classes specialize to classes in $H^{\bullet}\left(G_{K}, V\right)$ and can thus be regarded as characteristic classes of curves $C$ over $K$ with $n$ known rational points. They should be useful in investigating whether or not $C$ has any additional $K$-rational points.

Convention: Suppose that $k$ is a field. By a $k$-variety we will mean an integral, separated scheme of finite type over $k$. All $k$-schemes will be integral. By a family of smooth varieties over $X$ we mean a flat morphism $X \rightarrow T$, each of whose closed fibers is smooth. When we want to emphasize that $\mathcal{M}_{g, n}$ is regarded as a stack over Spec $k$, we will denote it by the unfortunately cumbersome notation $\mathcal{M}_{g, n / k}$.

\section{Fundamental groups and Galois groups}

We will have occasion to use various fundamental groups. In this section we enumerate some of them and summarize their basic properties. Unless mentioned otherwise, throughout this section $X$ is a smooth, geometrically connected variety defined over a field $k$ of characteristic zero.

2.1. The topological fundamental group. When $k$ is a subfield of $\mathbb{C}$, we shall denote the corresponding analytic variety by $X^{\text {an }}$. For $x \in X(\mathbb{C})$, we have the topological fundamental group $\pi_{1}^{\text {top }}\left(X^{\text {an }}, x\right)$, which we shall denote simply by $\pi_{1}^{\text {top }}(X, x)$.

If $X$ is a DM stack over $k$, the associated analytic space $X^{\text {an }}$ is an orbifold (i.e., a stack in the category of topological spaces). In this case, we will denote the orbifold fundamental group of $X^{\text {an }}$ by $\pi_{1}^{\text {orb }}(X, x)$, where $x$ is any suitable basepoint. Such fundamental groups are defined in [36, for example. Typically in this paper, $X$ will be the quotient of a smooth variety by a finite group. In this case the orbifold fundamental group is easy to describe directly. See [17, §3], for example.

2.2. The étale fundamental group. Denote the étale fundamental group of $X$ by $\pi_{1}(X, \bar{\eta})$, where $\bar{\eta}$ is a geometric point of $X$. The étale fundamental group of a DM stack $X$ can also be defined; see [36]. We will also denote it by $\pi_{1}(X, \bar{\eta})$. Fix an algebraic closure $\bar{k}$ of $k$. The fundamental group of Spec $k$ with respect to the geometric point $\operatorname{Spec} \bar{k} \rightarrow \operatorname{Spec} k$ is simply $\operatorname{Gal}(\bar{k} / k)$. We denote it by $G_{k}$. The structure morphism $X \rightarrow$ Spec $k$ induces a homomorphism $\pi_{1}(X, \bar{\eta}) \rightarrow G_{k}$. One has the canonical short exact sequence

$$
1 \rightarrow \pi_{1}\left(X \otimes_{k} \bar{k}, \bar{\eta}\right) \rightarrow \pi_{1}(X, \bar{\eta}) \rightarrow G_{k} \rightarrow 1 .
$$

Each $k$-rational point $x \in X(k)$ induces a section of $\pi_{1}(X, \bar{\eta}) \rightarrow G_{k}$ that is well defined up to conjugation by an element of the geometric fundamental group $\pi_{1}\left(X \otimes_{k} \bar{k}, \bar{\eta}\right)$. 
2.3. Topological versus étale fundamental groups. When $k=\mathbb{C}$ and $x \in$ $X(\mathbb{C})$, there is a natural isomorphism

$$
\pi_{1}(X, x) \cong \pi_{1}^{\mathrm{top}}(X, x)^{\wedge}
$$

between the étale fundamental group of $X$ and the profinite completion of its topological fundamental group. When $k$ is a subfield of $\mathbb{C}$, the exact sequence (2) becomes

$$
1 \rightarrow \pi_{1}^{\mathrm{top}}(X, x)^{\wedge} \rightarrow \pi_{1}(X, x) \rightarrow G_{k} \rightarrow 1 .
$$

2.4. Relation to Galois groups. Set $K=k(X)$ and let $\bar{\eta}$ be any geometric point that lies over the generic point of $X$. This also serves as a basepoint for any Zariski open subset of $X$. We therefore have an inverse system of profinite groups

$$
\left\{\pi_{1}(X-D, \bar{\eta})\right\}_{D},
$$

where $D$ ranges over the divisors in $X$ that are defined over $k$.

Denote the Galois group $\operatorname{Gal}(\bar{K} / K)$ of $K$ by $G_{K}$. There are natural surjections

$$
G_{K} \rightarrow \pi_{1}(X-D, \bar{\eta})
$$

for each divisor $D$ in $X$.

Proposition 2.1. The natural homomorphism

$$
G_{K} \rightarrow \underset{D}{\lim _{D}} \pi_{1}(X-D, \bar{\eta})
$$

is an isomorphism.

\section{Points And SECTIONS}

Suppose that $T$ is a smooth variety over $k$, a field of characteristic zero, and that $f: C \rightarrow T$ is a family of smooth projective curves. That is, $f$ is a proper flat family of geometrically connected smooth projective curves over $T$. Denote the function field $k(T)$ of $T$ by $K$. The following assertion follows directly from the valuative criterion for properness [11, 7.3.8]. It can also be proved by an elementary direct argument.

Proposition 3.1. There is a natural 1-1 correspondence between $K$-rational points $x \in C(K)$ and equivalence classes of sections $s: U \rightarrow C$ of $f$ defined on a Zariski open subset $U$ of $T$ defined over $k$, where each component of $T-U$ has codimension $\geq 2$ in $T$. Two such sections are defined to be equivalent if they agree on the intersection of their domains.

Fix a prime number $\ell$ and let $\# \in\{$ ét, top $\}$ if $k=\mathbb{C}$ and $\#=$ ét in general. Fix geometric points $t_{o}$ of $T$ and $x_{o}$ of $C$ such that $f\left(x_{o}\right)=t_{o}$. Denote the fiber of $C$ over $t_{o}$ by $C_{o}$.

Corollary 3.2. Each $x \in C(K)$ induces a splitting $x_{*}: \pi_{1}^{\#}\left(T, t_{o}\right) \rightarrow \pi_{1}^{\#}\left(C, x_{o}\right)$ of the natural homomorphism $f_{*}: \pi_{1}^{\#}\left(C, x_{o}\right) \rightarrow \pi_{1}^{\#}\left(T, t_{o}\right)$ that is unique up to conjugation by an element of $\pi_{1}^{\#}\left(C_{o}, x_{o}\right)$.

Remark 3.3. A result of Stix 42 implies that rational points $x \in C(K)$ correspond to global sections of $C \rightarrow T$. When $k=\mathbb{C}$, this follows from Teichmüller theory. Indeed, if $s: U \rightarrow C$ is a holomorphic section $C \rightarrow T$ defined in the complement of an analytic subset $Z$ of $T$, each of whose components has codimension $\geq 2$, then the inclusion $U \hookrightarrow T$ induces an isomorphism on fundamental groups, and thus 
an inclusion of universal coverings $\widetilde{U} \hookrightarrow \widetilde{T}$. The section $s$ lifts to a holomorphic mapping $\tilde{s}: \widetilde{U} \rightarrow \mathcal{X}_{g, 1}$ into the Teichmüller space $\mathcal{X}_{g, 1}$ of marked Riemann surfaces of type $(g, 1)$. Since $\mathcal{X}_{g, 1}$ is a domain in $\mathbb{C}^{3 g-2}$, Hartog's Theorem implies that $\tilde{s}$ extends to a holomorphic mapping $\widetilde{T} \rightarrow \mathcal{X}_{g, 1}$, which implies that $s$ extends to a holomorphic section of $C \rightarrow T$.

\section{Monodromy Representations}

4.1. The groups $\operatorname{Sp}(H)$ and $\operatorname{GSp}(H)$. Suppose that $g \geq 1$ and that $A$ is a commutative ring. Suppose that $H_{A}$ is a free $A$-module of rank $2 g$ endowed with a unimodular, skew-symmetric bilinear form

$$
\beta: H_{A} \otimes H_{A} \rightarrow A .
$$

For an $A$-algebra $R$, set $H_{R}=H_{A} \otimes_{A} R$. The general symplectic group is defined by

$$
\operatorname{GSp}\left(H_{R}\right):=\left\{\phi: H_{R} \rightarrow H_{R}: \phi^{*} \beta=\tau(\phi) \beta \text { for some } \tau(\phi) \in R^{\times}\right\} .
$$

Taking $\phi$ to $\tau(\phi)$ defines a homomorphism $\tau: \operatorname{GSp}\left(H_{R}\right) \rightarrow R^{\times}$whose kernel is the symplectic group $\operatorname{Sp}\left(H_{R}\right)$.

We will view $\operatorname{GSp}\left(H_{A}\right)$ and $\operatorname{Sp}\left(H_{A}\right)$ as group schemes over $A$ whose groups of $R$-rational points are $\operatorname{GSp}\left(H_{R}\right)$ and $\operatorname{Sp}\left(H_{R}\right)$, respectively. We will omit the ground ring $A$ from the notation when it should be clear from the context. There is an exact sequence of algebraic groups

$$
1 \rightarrow \mathrm{Sp}(H) \rightarrow \mathrm{GSp}(H) \stackrel{\tau}{\rightarrow} \mathbb{G}_{m} \rightarrow 1 .
$$

The pairing $\beta$ can be replaced by a $\operatorname{GSp}(H)$-pairing $\theta$ as follows. For $r \in \mathbb{Z}$, denote by $A(r)$ the representation of $\operatorname{GSp}(H)$ whose underlying $A$-module is free of rank 1 and where $\operatorname{GSp}\left(H_{A}\right)$ acts via $\tau^{r}$. Each choice of a generator $a_{o}$ of $A(1)$ gives rise to the $\operatorname{GSp}(H)$-invariant bilinear pairing

$$
\theta: H \otimes H \rightarrow A(1), \quad \theta: x \otimes y \mapsto \beta(x, y) a_{o} .
$$

With this choice,

$$
\operatorname{GSp}\left(H_{R}\right):=\left\{\phi: H_{R} \rightarrow H_{R}: \phi^{*} \theta=\theta\right\} .
$$

For a $\operatorname{GSp}\left(H_{A}\right)$-module $V$, denote by $V(r)$ the representation $V \otimes_{A} A(r)$. Since $\beta$ is unimodular, $\theta$ induces an isomorphism

$$
H_{A} \stackrel{\simeq}{\longrightarrow} H_{A}^{*}(1):=\operatorname{Hom}_{A}\left(H_{A}, A\right)(1) .
$$

The representation $\Lambda^{2} V$ will be regarded as a quotient of $V^{\otimes 2}$; the image of $v_{1} \otimes v_{2}$ will be denoted $v_{1} \wedge v_{2}$. The pairing

$$
\left(\Lambda^{2} H_{A}^{*}\right) \otimes\left(\Lambda^{2} H_{A}\right) \rightarrow A, \quad\left(\phi_{1} \wedge \phi_{2}\right) \otimes\left(x_{1} \wedge x_{2}\right):=\operatorname{det}\left(\phi_{i}\left(x_{j}\right)\right)
$$

is perfect and thus induces isomorphisms

$$
\left(\Lambda^{2} H_{A}\right)^{*}(2) \cong \Lambda^{2}\left(H_{A}^{*}(1)\right) \cong \Lambda^{2} H_{A} .
$$

The dual pairing $\check{\theta}: H_{A}^{*} \otimes H_{A}^{*} \rightarrow A(-1)$ will be regarded as a $\operatorname{GSp}(H)$-equivariant map $\check{\theta}: A(1) \rightarrow \Lambda^{2} H_{A}$ or, equivalently, as an element of $\Lambda^{2} H_{A}(-1)$. 
4.2. Monodromy representations. Suppose that $T$ is a smooth variety over a field $k$ of characteristic zero and that $f: C \rightarrow T$ is a family of smooth projective curves over $T$. Fix a geometric point $\bar{\eta}: \operatorname{Spec} F \rightarrow T$ of $T$ and denote the fiber of $C$ over it by $C_{\bar{\eta}}$. For a prime number $\ell$ we set

$$
H_{\mathbb{Z}_{\ell}}=H_{\text {ét }}^{1}\left(C_{\bar{\eta}}, \mathbb{Z}_{\ell}(1)\right) .
$$

This is endowed with the cup product pairing $\theta: \Lambda^{2} H_{\mathbb{Z}_{\ell}} \rightarrow \mathbb{Z}_{\ell}(1)$, which is unimodular. Let $\bar{x}$ be a geometric point of $C$ that lies over $\bar{\eta}$.

Lemma 4.1. If $g \geq 2$ or if $g=1$ and $f: C \rightarrow T$ has a section, then the homomorphism $\pi_{1}\left(C_{\bar{\eta}}, \bar{x}\right) \rightarrow \pi_{1}(C, \bar{x})$ induced by $C_{\bar{\eta}} \rightarrow C$ is injective.

Proof. The result is clear when $f$ has a section. So suppose that $g \geq 2$. Since $\pi_{1}(C \otimes \bar{k}, \bar{x}) \rightarrow \pi_{1}(C, \bar{x})$ is injective, it suffices to consider the case where $k$ is algebraically closed. We may assume that $k=\mathbb{C}$ and that $\bar{\eta} \in T(\mathbb{C})$ and $\bar{x} \in C(\mathbb{C})$. Standard algebraic topology implies that $\pi_{1}^{\text {top }}(C, \bar{x})$ acts on $\pi_{1}^{\text {top }}\left(C_{\bar{\eta}}, \bar{x}\right)$ and that the composite

$$
\pi_{1}^{\mathrm{top}}\left(C_{\bar{\eta}}, \bar{x}\right) \rightarrow \pi_{1}^{\mathrm{top}}(C, \bar{x}) \rightarrow \operatorname{Aut}\left(\pi_{1}^{\mathrm{top}}\left(C_{\bar{\eta}}, \bar{x}\right)\right)
$$

is the conjugation action. Taking profinite completions, it follows that there is a continuous action of $\pi_{1}(C, \bar{x})$ on $\pi_{1}\left(C_{\bar{\eta}}, \bar{x}\right)$ and that

$$
\pi_{1}\left(C_{\bar{\eta}}, \bar{x}\right) \rightarrow \pi_{1}(C, \bar{x}) \rightarrow \operatorname{Aut}\left(\pi_{1}\left(C_{\bar{\eta}}, \bar{x}\right)\right)
$$

is the conjugation action. Since $\pi_{1}\left(C_{\bar{\eta}}, \bar{x}\right)$ has trivial center [1, it follows that $\pi_{1}\left(C_{\bar{\eta}}, \bar{x}\right) \rightarrow \pi_{1}(C, \bar{x})$ is injective.

Suppose that $g \geq 2$ or that $g=1$ and that $f$ has a section. Since $H_{\mathbb{Z}_{\ell}} \cong$ $\operatorname{Hom}\left(\pi_{1}\left(C_{\bar{\eta}}, \bar{x}\right), \mathbb{Z}_{\ell}(1)\right)$, the conjugation action of $\pi_{1}(C, \bar{x})$ on $\pi_{1}\left(C_{\bar{\eta}}, \bar{x}\right)$ induces a natural monodromy action

$$
\rho_{\bar{\eta}}: \pi_{1}(T, \bar{\eta}) \rightarrow \operatorname{GSp}\left(H_{\mathbb{Z}_{\ell}}\right)
$$

such that the diagram

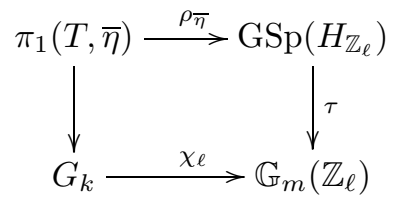

commutes, where the left-hand map is the canonical projection, the right-hand vertical map $\tau$ is the natural surjection, and where $\chi_{\ell}$ is the $\ell$-adic cyclotomic character. Often we will extend scalars from $\mathbb{Z}_{\ell}$ to $\mathbb{Q}_{\ell}$.

Denote the projection by $f: C \rightarrow T$. For $A=\mathbb{Z}_{\ell}$ or $\mathbb{Q}_{\ell}$, set

$$
\mathbb{H}_{A}:=R^{1} f_{*} A(1) \text {. }
$$

Proposition 4.2. If $g \geq 2$ or if $g=1$ and $f: C \rightarrow T$ has a section, then the monodromy representation of $\pi_{1}(T, \bar{\eta})$ on the fiber $H_{A}$ of $\mathbb{H}_{A}$ over $\bar{\eta}$ is $\rho_{\bar{\eta}}$ : $\pi_{1}(T, \bar{\eta}) \rightarrow$ Aut $H_{A}$.

Proof. The proof of the topological analogue of the assertion is an exercise in topology and is left to the reader 3 We prove the arithmetic version. Let $K=k(T)$. Fix

\footnotetext{
${ }^{3}$ Reduce to the case of a mapping torus.
} 
an algebraic closure $\bar{K}$ of $K$. We may assume that $\bar{\eta}=\operatorname{Spec} \bar{K}$. Then one has the diagram

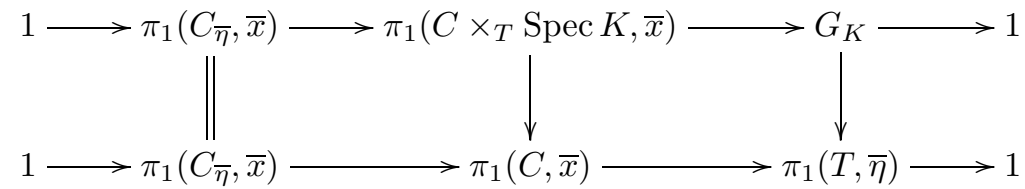

in which the vertical maps are surjective. The Galois action $G_{K} \rightarrow$ Aut $H_{A}$ is induced by the conjugation action of $\pi_{1}\left(C \times_{T}\right.$ Spec $\left.K, \bar{x}\right)$ on $\pi_{1}\left(C_{\bar{\eta}}, \bar{x}\right)$. The result now follows from naturality of the monodromy action under base change and the commutativity of the diagram.

\section{MAPPing Class groups AND MOdUli STACKS OF CURVES}

Suppose that $g$ and $n$ are non-negative integers satisfying $2 g-2+n>0$. Fix a closed, oriented surface $S$ of genus $g$ and a finite subset $\mathbf{x}=\left\{x_{1}, \ldots, x_{n}\right\}$ of $n$ distinct points in $S$. The corresponding mapping class group will be denoted

$$
\Gamma_{S, \mathbf{x}}=\pi_{0} \operatorname{Diff}^{+}(S, \mathbf{x}),
$$

where $\operatorname{Diff}^{+}(S, \mathbf{x})$ denotes the group of orientation-preserving diffeomorphisms of $S$ that fix x pointwise. By the classification of surfaces, the diffeomorphism class of $(S, \mathbf{x})$ depends only on $(g, n)$. Consequently, the group $\Gamma_{S, \mathbf{x}}$ depends only on the pair $(g, n)$. It will be denoted by $\Gamma_{g, n}$ when we have no particular marked surface $(S, \mathbf{x})$ in mind.

Denote the moduli stack over Spec $k$ of smooth projective curves of genus $g$ with $n$ distinct marked points by $\mathcal{M}_{g, n / k}$. The corresponding complex analytic orbifold will be denoted by $\mathcal{M}_{g, n}^{\text {an }}$. Recall that $\pi_{1}^{\text {orb }}\left(\mathcal{M}_{g, n}, \eta\right)$ denotes the orbifold fundamental group of $\mathcal{M}_{g, n}^{\text {an }}$.

If $(C, \mathbf{x})$ is an $n$-pointed smooth complex projective curve, there is a natural isomorphism

$$
\pi_{1}^{\text {orb }}\left(\mathcal{M}_{g, n},[C, \mathbf{x}]\right) \cong \Gamma_{C, \mathbf{x}} .
$$

For each geometric point $\bar{\eta}$ of $\mathcal{M}_{g, n / k}$ there is therefore an isomorphism

$$
\pi_{1}\left(\mathcal{M}_{g, n / \bar{k}}, \bar{\eta}\right) \cong \Gamma_{g, n}^{\wedge}
$$

which is well defined up to inner automorphisms, and an exact sequence

$$
1 \rightarrow \Gamma_{g, n}^{\wedge} \rightarrow \pi_{1}\left(\mathcal{M}_{g, n / k}, \bar{\eta}\right) \rightarrow G_{k} \rightarrow 1 .
$$

The Lefschetz trace formula implies that when $n>2 g+2$, the automorphism group of each $n$-marked, genus $g$ curve is trivial. In this case, $\mathcal{M}_{g, n / k}$ is a quasiprojective $k$-scheme, 31]. When $n \leq 2 g+2$, we can replace $\mathcal{M}_{g, n}$ by $\mathcal{M}_{g, n}$, the locus of $n$-pointed genus $g$ curves in $\mathcal{M}_{g, n}$ with trivial automorphism group. Since the locus of $n$-pointed curves in $\mathcal{M}_{g, n}$ with a non-trivial automorphism is a closed subscheme of $\mathcal{M}_{g, n}$, Knudsen's result 31 implies that $\mathcal{M}_{g, n}$ is a smooth $k$-scheme.

Proposition 5.1. If $g \geq 2$, then each component of the complement of $\mathcal{M}_{g, n}$ in $\mathcal{M}_{g, n}$ has codimension $\geq g+n-2$. Consequently, if $g+n \geq 4$, the inclusion $\mathcal{M}_{g, n} \rightarrow$ $\mathcal{M}_{g, n}$ induces an isomorphism $\pi_{1}^{\mathrm{top}}\left(\mathcal{M}_{g, n}, x\right) \cong \Gamma_{g, n}$ of fundamental groups. 
Sketch of proof. We will prove the case $n=0$. The case $n>0$ is an immediate consequence as marking points on a curve $C$ cannot create new automorphisms, only kill them. First note that if a curve has a non-trivial automorphism, it has one of prime order. Suppose that $S$ is a compact oriented surface of genus $g$ and that $G$ is a finite cyclic group of prime order $p$ that acts effectively on $S$ and preserves the orientation. Since the stabilizer of each point is cyclic, $G \backslash S$ is a compact surface and the projection $\pi: S \rightarrow G \backslash S$ is a branched covering. Let $h$ be the genus of $G \backslash S$.

The Riemann-Hurwicz formula implies that

$$
h-1=\frac{g-1}{p}-\frac{1}{2} \sum_{y \in A}\left(1-\frac{1}{p}\right),
$$

where $A$ is the set of critical values of $\pi$. Let $a=|A|$. Teichmüller theory implies that the locus of curves in $\mathcal{M}_{g}$, where $G \subseteq$ Aut $C$ and the action of $G$ on $C$ is conjugate to the given action of $G$ on $S$, is the image of a morphism $\mathcal{M}_{h, a} \rightarrow \mathcal{M}_{g}$. This locus has codimension

$$
\operatorname{dim} \mathcal{M}_{g}-\operatorname{dim} \mathcal{M}_{h, a}=(3 g-3)-(3 h-3+a)
$$

as $g \geq 2$ implies that $(h, a)$ cannot be $(1,0)$. Equation (3) implies that the codimension of this locus is

$$
\left(3 g-3+\left(\frac{3}{2}-\frac{1}{1-p^{-1}}\right) a\right)(1-1 / p) .
$$

When $p=2$ the codimension is $(3 g-3-a / 2) / 2$, which is $\geq g-2$ as $a \leq 2 g+2$ with equality if and only if $a=2 g+2$ and $h=0$, in which case $C$ is hyperelliptic. When $p \geq 3$, then $3 / 2 \geq\left(1-p^{-1}\right)^{-1}$. In this case,

$$
\operatorname{codim} \geq(3 g-3)\left(1-p^{-1}\right) \geq 2 g-2
$$

with equality if and only if $a=0$ and $p=3$.

\subsection{Level structures.}

5.1.1. In topology. Suppose that $S$ is a compact oriented reference surface of genus g. Set $H_{\mathbb{Z}}=H_{1}(S, \mathbb{Z})$ endowed with its intersection pairing. For a positive integer $m$, the level $m$ subgroup $\operatorname{Sp}\left(H_{\mathbb{Z}}\right)[m]$ of $\operatorname{Sp}\left(H_{\mathbb{Z}}\right)$ is defined to be the kernel of the quotient homomorphism

$$
\operatorname{Sp}\left(H_{\mathbb{Z}}\right) \rightarrow \operatorname{Sp}\left(H_{\mathbb{Z} / m \mathbb{Z}}\right) .
$$

The group $\operatorname{Sp}\left(H_{\mathbb{Z}}\right)[m]$ is torsion free for all $m \geq 3$.

The action of $\operatorname{Diff}^{+}(S, \mathbf{x})$ on $S$ induces a surjective homomorphism

$$
\rho: \Gamma_{S, \mathbf{x}} \rightarrow \operatorname{Sp}\left(H_{\mathbb{Z}}\right) .
$$

Define the level $m$ subgroup of $\Gamma_{g, n}$ to be the kernel of the composite:

$$
\Gamma_{g, n} \rightarrow \Gamma_{g} \rightarrow \operatorname{Sp}\left(H_{\mathbb{Z} / m \mathbb{Z})} .\right.
$$

Denote it by $\Gamma_{g, n}[m]$. It is torsion free when $m \geq 3$. 
5.1.2. In arithmetic. Suppose that $m$ is a positive integer and that $k$ is a field of characteristic zero that contains all $m$ th roots of unity $\boldsymbol{\mu}_{m}(\bar{k})$. Fix an isomorphism $\psi: \operatorname{Hom}\left(\boldsymbol{\mu}_{m}(\bar{k}), \mathbb{Q} / \mathbb{Z}\right) \rightarrow \mathbb{Z} / m \mathbb{Z}$. Such isomorphisms correspond to choices of a primitive $m$ th root of unity. A level $m$ structure on a smooth projective curve $C$ of genus $g$ over $k$ is an isomorphism

$$
\phi: H_{\text {ét }}^{1}\left(C \otimes_{k} \bar{k}, \mathbb{Z} / m \mathbb{Z}\right) \rightarrow(\mathbb{Z} / m \mathbb{Z})^{2 g}
$$

such that the diagram

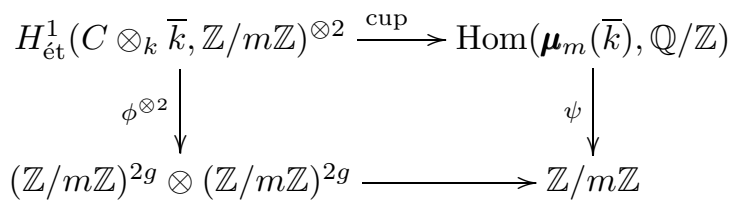

commutes, where the bottom row is the standard symplectic inner product on $(\mathbb{Z} / m \mathbb{Z})^{2 g}$.

When considering the moduli of curves with a level $m$ structure over $k$, we will assume, as above, that $k$ contains the $m$ th roots of unity $\boldsymbol{\mu}_{m}(\bar{k})$ and that the isomorphism $\psi$ is specified. This ensures that the moduli stack $\mathcal{M}_{g, n / k}[m]$ is geometrically connected. It is a principal $\operatorname{Sp}_{g}(\mathbb{Z} / m \mathbb{Z})$-bundle over $\mathcal{M}_{g, n / k}$. When $m \geq 3$, $\mathcal{M}_{g, n}[m]$ is a smooth quasi-projective variety. This follows from [33, Thm. 1.10] using the GIT setup from [9, $\S 2]$.

When $k$ is an algebraically closed subfield of $\mathbb{C}$, there is a natural conjugacy class of isomorphisms $\pi_{1}^{\text {orb }}\left(\mathcal{M}_{g, n}[m], *\right) \cong \Gamma_{g, n}[m]$.

\section{Relative And Weighted COMPletion of PROFinite GRoups}

This section is a terse review of the relative and weighted completions of profinite groups. The reader is referred to the surveys [21, 16], and the articles [13, 15, 20] for a more detailed discussion and details.

6.1. Relative completion of a discrete group. Suppose that $\Gamma$ is a discrete group and that $R$ is a reductive $F$-group, where $F$ is a field of characteristic zero (the coefficient field). The completion of $\Gamma$ with respect to a Zariski dense representation $\rho: \Gamma \rightarrow R(F)$ is a proalgebraic $F$-group $\mathcal{G}$, which is an extension

$$
1 \rightarrow \mathcal{U} \rightarrow \mathcal{G} \rightarrow R \rightarrow 1
$$

of $R$ by a prounipotent group $\mathcal{U}$, and a Zariski dense representation $\tilde{\rho}: \Gamma \rightarrow \mathcal{G}(F)$ whose composition with $\mathcal{G}(F) \rightarrow R(F)$ is $\rho$. It is characterized by the following universal mapping property: if $G$ is a proalgebraic $F$-group that is an extension

$$
1 \rightarrow U \rightarrow G \rightarrow R \rightarrow 1
$$

of $R$ by a unipotent $F$-group $U$, and if $\phi: \Gamma \rightarrow G(F)$ is a homomorphism whose composition with $G(F) \rightarrow R(F)$ is $\rho$, then there is a homomorphism $\mathcal{G} \rightarrow G$ of $F$ groups that commutes with the projections to $R$ and such that $\phi$ is the composite $\Gamma \rightarrow \mathcal{G}(F) \rightarrow G(F)$.

When $R$ is the trivial group, $\mathcal{G}=\mathcal{U}$, which is a prounipotent group. In this case, $\mathcal{U}$ and $\Gamma \rightarrow \mathcal{U}(F)$ comprise the unipotent completion of $\Gamma$ over $F$. It will be denoted by $\Gamma_{/ F}^{u n}$.

A more interesting example is where $\Gamma$ is the mapping class group $\Gamma_{g, n}$, where $2 g-2+n>0$ and $g \geq 1$. In this case, $\Gamma_{g, n / F}^{u n}$ is trivial. A better choice is to take 
$R=\operatorname{Sp}\left(H_{\mathbb{Q}}\right)$, where $H_{A}=H_{1}(S, A)$ is the first homology of the compact reference surface $S$, and take $\rho: \Gamma_{g, n} \rightarrow \operatorname{Sp}\left(H_{\mathbb{Q}}\right)$ to be the representation of the mapping class group on the first homology of the surface. Since this has image $\operatorname{Sp}\left(H_{\mathbb{Z}}\right)$, it is Zariski dense. Denote the completion of $\Gamma_{g, n}$ with respect to $\rho$ by $\mathcal{G}_{g, n}^{\text {geom }}$ and its prounipotent radical by $\mathcal{U}_{g, n}^{\text {geom }}$. These are proalgebraic $\mathbb{Q}$-groups.

One can restrict the homomorphism $\Gamma_{g, n} \rightarrow \mathcal{G}_{g, n}^{\text {geom }}(\mathbb{Q})$ to the level $m$ subgroup.

Proposition 6.1 ([15, Prop. 3.3]). If $g \geq 3$, then for all $m \geq 1$, the group $\mathcal{G}_{g, n}^{\text {geom }}$ with the homomorphism $\Gamma_{g, n}[\mathrm{~m}] \hookrightarrow \Gamma_{g, n} \rightarrow \mathcal{G}_{g, n}^{\text {geom }}(\mathbb{Q})$ is the completion of $\Gamma_{g, n}[\mathrm{~m}]$ relative to the natural homomorphism $\Gamma_{g, n}[m] \rightarrow \mathrm{Sp}\left(H_{\mathbb{Q}}\right)$.

We have been vague about the role played by the coefficient field $F$. This is because relative completion behaves well under base change.

Theorem $6.2([23])$. Assume the notation of this subsection. If $E$ is an extension field of $F$ and if the image of $\rho: \Gamma \rightarrow R(E)$ is Zariski dense in $R \otimes_{F} E$, then $\mathcal{G} \otimes_{F} E$ and $\rho_{E}: \Gamma \rightarrow R(E)=\left(R \otimes_{F} E\right)(E)$ comprise the completion of $\Gamma$ relative to $\rho_{E}$.

This is proved directly for relative completions of mapping class groups in 15, Thm. 3.1]. It implies that the completion of $\Gamma_{g, n}[m]$ relative to $\rho: \Gamma_{g, n}[m] \rightarrow$ $\operatorname{Sp}\left(H_{\mathbb{Q}_{\ell}}\right)$ is $\mathcal{G}_{g, n}^{\text {geom }} \otimes_{\mathbb{Q}} \mathbb{Q}_{\ell}$.

6.2. Continuous relative completion of a profinite group. This is the profinite analogue of relative completion of a discrete group. In this case, we take the coefficient field $F$ to be the field $\mathbb{Q}_{\ell}$ for some prime number $\ell$. Suppose that $\Gamma$ is a profinite group, that $R$ is a reductive $\mathbb{Q}_{\ell}$-group, and that $\rho: \Gamma \rightarrow R\left(\mathbb{Q}_{\ell}\right)$ is a continuous, Zariski dense representation. The continuous completion of $\Gamma$ with respect to $\rho$ is a proalgebraic $\mathbb{Q}_{\ell}$-group $\mathcal{G}$ that is an extension

$$
1 \rightarrow \mathcal{U} \rightarrow \mathcal{G} \rightarrow R \rightarrow 1
$$

of $R$ by a prounipotent $\mathbb{Q}_{\ell}$-group $\mathcal{U}$ and a continuous 4 Zariski dense representation $\tilde{\rho}: \Gamma \rightarrow \mathcal{G}\left(\mathbb{Q}_{\ell}\right)$ whose composition with $\mathcal{G}\left(\mathbb{Q}_{\ell}\right) \rightarrow R\left(\mathbb{Q}_{\ell}\right)$ is $\rho$. It is characterized by a universal mapping property that is similar to the one that characterizes the relative completion of a discrete group. The only difference is that all homomorphisms are required to be continuous in the $\ell$-adic profinite case.

When $R$ is the trivial group, this reduces to the continuous unipotent completion of a profinite group, [20, A.2].

The continuous relative completion of the profinite completion of a discrete group can be computed from the relative completion of the discrete group. Denote the profinite completion of the discrete group $\Gamma$ by $\Gamma^{\wedge}$. We can view $\Gamma$ as a topological group by defining the neighbourhoods of the identity to be the finite index normal subgroups of $\Gamma$.

Theorem 6.3 (20, 23]). Suppose that $\Gamma$ is a discrete group, $R$ is a reductive $\mathbb{Q}_{\ell-}$ group, and that $\rho: \Gamma \rightarrow R\left(\mathbb{Q}_{\ell}\right)$ is a continuous, Zariski dense representation. Let $\rho_{\ell}: \Gamma^{\wedge} \rightarrow R\left(\mathbb{Q}_{\ell}\right)$ be the continuous extension of $\rho$ to $\Gamma^{\wedge}$. If $\mathcal{G}$ and $\tilde{\rho}: \Gamma \rightarrow \mathcal{G}\left(\mathbb{Q}_{\ell}\right)$

\footnotetext{
${ }^{4}$ A homomorphism $\phi: \Gamma \rightarrow G\left(\mathbb{Q}_{\ell}\right)$ from a profinite group to the rational points of a proalgebraic $\mathbb{Q}_{\ell}$-group $G$ is defined to be continuous if it is the inverse limit of continuous homomorphisms $\Gamma \rightarrow G_{\alpha}\left(\mathbb{Q}_{\ell}\right)$, where each $G_{\alpha}$ is an algebraic $\mathbb{Q}_{\ell}$-group and $G=\lim _{\longleftarrow} G_{\alpha}$.
} 
is the completion of $\Gamma$ relative to $\rho$, then:

(i) $\tilde{\rho}$ is continuous and thus induces a continuous homomorphism $\hat{\rho}_{\ell}: \Gamma^{\wedge} \rightarrow$ $\mathcal{G}\left(\mathbb{Q}_{\ell}\right) ;$

(ii) $\mathcal{G}$ and $\hat{\rho}_{\ell}$ form the continuous relative completion of $\Gamma^{\wedge}$ with respect to $\rho_{\ell}$.

In particular, the continuous completion of $\Gamma_{g, n}^{\wedge}[m]$ with respect to the standard representation $\rho_{\ell}: \Gamma_{g, n}^{\wedge}[m] \rightarrow \operatorname{Sp}\left(H_{\mathbb{Q}_{\ell}}\right)$ is $\mathcal{G}_{g, n}^{\text {geom }} \otimes_{\mathbb{Q}} \mathbb{Q}_{\ell}$. It also implies that the continuous unipotent completion of the profinite completion $\pi^{\wedge}$ of the topological fundamental group $\pi$ of an $n$-punctured, genus $g$ surface is $\pi_{/ \mathbb{Q}_{\ell}}^{\text {un }}$.

6.3. Negatively weighted representations. Suppose that $F$ is a field of characteristic zero. Denote $\mathbb{G}_{m / F}$ by $\mathbb{G}_{m}$. First suppose that $R$ is a reductive $F$-group and that $\omega: \mathbb{G}_{m} \rightarrow R$ is a central cocharacter. If $V$ is an irreducible $F$-rational representation of $R$, then the restriction of $V$ to $\mathbb{G}_{m}$ via $\omega$ is isotypical. That is, there is an integer $w(V)$ such that $\mathbb{G}_{m}$ acts on $V$ via the $w(V)$ th power of its defining representation. We will call $w(V)$ the weight of $V$ (with respect to $\omega$ ) and say that $V$ has negative weight when $w(V)<0$. More generally, a finite dimensional representation of $R$ is negatively weighted when each of its irreducible components is.

We will say that an extension

$$
1 \rightarrow U \rightarrow G \rightarrow R \rightarrow 1
$$

of affine algebraic $F$-groups, where $U$ is unipotent, is negatively weighted (with respect to $\omega$ ) when the abelianization $H_{1}(U)$ of $U$ is a negatively weighted $R$ module. An extension of $R$ by a prounipotent $F$-group $U$ is negatively weighted if it is the inverse limit of negatively weighted extensions of $R$ by unipotent groups.

Proposition 6.4 ([20, Thms. 3.9 \& 3.12]). Suppose that $R$ is a reductive F-group and that $\omega: \mathbb{G}_{m} \rightarrow R$ is a central cocharacter. If $G$ is a proalgebraic group that is a negatively weighted extension (with respect to $\omega$ ) of $R$ by a prounipotent group, then every finite dimensional $G$-module $V$ has a natural weight filtration $W_{\bullet}$ :

$$
0=W_{N} V \subseteq \cdots \subseteq W_{r-1} V \subseteq W_{r} V \subseteq \cdots \subseteq W_{M} V=V .
$$

It is characterized by the property that the action of $G$ on

$$
\operatorname{Gr}_{r}^{W} V:=W_{r} V / W_{r-1} V
$$

factors through $G \rightarrow R$ and is a representation of $R$ of weight $r$. The weight filtration is preserved by $G$-module homomorphisms, and the functor $\mathrm{Gr}_{\bullet}^{W}$ on the category of $G$-modules is exact.

Remark 6.5 . Each choice of a lift $\tilde{\omega}: \mathbb{G}_{m} \rightarrow G$ of the central cocharacter $\omega: \mathbb{G}_{m} \rightarrow$ $R$ determines a vector space isomorphism $V \cong \mathrm{Gr}_{\bullet}^{W} V$ of a $G$-module $V$ with its associated weight graded space. This isomorphism is natural in the sense that the diagram of vector spaces

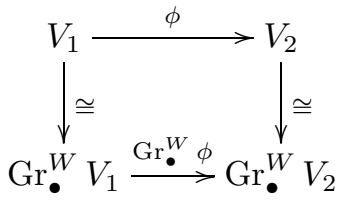


commutes for all $G$-module homomorphisms $\phi: V_{1} \rightarrow V_{2}$. Although the isomorphism $V \cong \mathrm{Gr}_{\bullet}^{W} V$ is natural, it is not canonical as it depends upon the choice of the lift $\tilde{\omega}$.

Denote the Lie algebras of $G, R$ and $U$ by $\mathfrak{g}, \mathfrak{r}$ and $\mathfrak{u}$, respectively.

Proposition 6.6 ([20, Prop. 4.5]). If $G$ is a proalgebraic F-group that is a negatively weighted extension of $R$ by a prounipotent group $U$, then the Lie algebra $\mathfrak{g}$ of $G$ and the Lie algebra $\mathfrak{u}$ of $U$ have natural weight filtrations $W_{\bullet}$ that satisfy

$$
\mathfrak{g}=W_{0} \mathfrak{g}, \quad \mathfrak{u}=W_{-1} \mathfrak{g}, \quad \mathfrak{r}=\operatorname{Gr}_{0}^{W} \mathfrak{g} .
$$

In particular, each choice of a lift $\tilde{\omega}: \mathbb{G}_{m} \rightarrow G$ of the central cocharacter $\omega: \mathbb{G}_{m} \rightarrow$ $R$ determines Lie algebra isomorphisms

$$
\mathfrak{g} \cong \prod_{r<0} \operatorname{Gr}_{r}^{W} \mathfrak{g} \text { and } \mathfrak{u} \cong \prod_{r<0} \operatorname{Gr}_{r}^{W} \mathfrak{u} .
$$

A pronilpotent Lie algebra $\mathfrak{n}$ in the category of $G$-modules is the inverse system $\left(\mathfrak{n}_{\alpha}\right)_{\alpha}$ of nilpotent Lie algebras in the category of $G$-modules. The continuous cohomology of a pronilpotent Lie algebra $\mathfrak{n}$ is defined by

$$
H^{\bullet}(\mathfrak{n})=\lim _{\longrightarrow} H^{\bullet}\left(\mathfrak{n}_{\alpha}\right)
$$

and its homology by

$$
H_{\bullet}(\mathfrak{n})=\lim _{\longleftarrow} H_{\bullet}\left(\mathfrak{n}_{\alpha}\right) .
$$

These are ind- and pro-objects, respectively, of the category of $G$-modules, and thus have natural weight filtrations. The latter can be regarded as a topological vector space under the inverse limit topology. There are natural $G$-module isomorphisms

$$
H^{\bullet}(\mathfrak{n}) \cong \operatorname{Hom}_{\mathrm{cts}}\left(H_{\bullet}(\mathfrak{n}), F\right) \text { and } H_{\bullet}(\mathfrak{n}) \cong \operatorname{Hom}\left(H^{\bullet}(\mathfrak{n}), F\right) .
$$

Exactness of $\mathrm{Gr}_{\bullet}^{W}$ implies that homology and cohomology commute with $\mathrm{Gr}_{\bullet}^{W}$. That is, there are canonical $R$-module isomorphisms

$$
H^{\bullet}\left(\mathrm{Gr}_{\bullet}^{W} \mathfrak{n}\right) \cong \mathrm{Gr}_{\bullet}^{W} H^{\bullet}(\mathfrak{n}) \text { and } H_{\bullet}\left(\mathrm{Gr}_{\bullet}^{W} \mathfrak{n}\right) \cong \mathrm{Gr}_{\bullet}^{W} H_{\bullet}(\mathfrak{n})
$$

The following useful result is an analogue of Sullivan's observation [43].

Lemma 6.7. Suppose that $G$ is a proalgebraic F-group that is a negatively weighted extension of $R$ by a prounipotent group $U$ and that $\mathfrak{n}$ is a pronilpotent Lie algebra in the category of $G$-modules. If $H_{1}(\mathfrak{n})$ has negative weights (i.e., $H_{1}(\mathfrak{n})=W_{-1} H_{1}(\mathfrak{n})$ ), then there is an $R$-module isomorphism $\mathrm{Gr}_{-1}^{W} \mathfrak{n}=\mathrm{Gr}_{-1}^{W} H_{1}(\mathfrak{n})$ and an exact sequence

$$
0 \rightarrow \mathrm{Gr}_{2}^{W} H^{1}(\mathfrak{n}) \rightarrow\left(\mathrm{Gr}_{-2}^{W} \mathfrak{n}\right)^{*} \stackrel{\text { bracket* }}{\longrightarrow} \Lambda^{2} \mathrm{Gr}_{1}^{W} H^{1}(\mathfrak{n}) \stackrel{\text { cup }}{\longrightarrow} \mathrm{Gr}_{2}^{W} H^{2}(\mathfrak{n}) \rightarrow 0
$$

in the category of ind-R-modules.

Proof. Denote the standard cochain complex of $\mathfrak{n}$ by $C^{\bullet}(\mathfrak{n})$, where

$$
C^{s}(\mathfrak{n})=\operatorname{Hom}_{\mathrm{cts}}\left(\Lambda^{s} \mathfrak{n}, F\right)
$$

Since $\mathfrak{n}=W_{-1} \mathfrak{n}$, this is a differential graded $F$-algebra in the category of ind$R$-modules. The exactness of $\mathrm{Gr}_{\bullet}^{W}$ implies that there are natural ind- $R$-module isomorphisms

$$
\mathrm{Gr}_{\bullet}^{W} H^{\bullet}(\mathfrak{n}) \cong \mathrm{Gr}_{\bullet}^{W} H^{\bullet}\left(C^{\bullet}(\mathfrak{n})\right) \cong H^{\bullet}\left(\mathrm{Gr}_{\bullet}^{W} C^{\bullet}(\mathfrak{n})\right)
$$


Since $H_{1}(\mathfrak{n})$ is negatively weighted, $\operatorname{Gr}_{1}^{W} C^{\bullet}(\mathfrak{n})$ is the complex $\operatorname{Hom}\left(\operatorname{Gr}_{-1}^{W} \mathfrak{n}, F\right)$, which implies that $\operatorname{Gr}_{1}^{W} H^{1}(\mathfrak{n})=\left(\mathrm{Gr}_{-1}^{W} \mathfrak{n}\right)^{*}$. Taking duals we see that

$$
\operatorname{Gr}_{-1}^{W} H_{1}(\mathfrak{n})=\left(\operatorname{Gr}_{1}^{W} H^{1}(\mathfrak{n})\right)^{*}=\operatorname{Gr}_{-1}^{W} \mathfrak{n} .
$$

The complex $\mathrm{Gr}_{-2}^{W} C^{\bullet}(\mathfrak{n})$ is the dual of

$$
\Lambda^{2} \mathrm{Gr}_{-1}^{W} \mathfrak{n} \stackrel{\text { bracket }}{\longrightarrow} \operatorname{Gr}_{-2}^{W} \mathfrak{n} .
$$

This and the isomorphism $\mathrm{Gr}_{1}^{W} H^{1}(\mathfrak{n})=\left(\mathrm{Gr}_{-1}^{W} \mathfrak{n}\right)^{*}$ imply the exactness of

$$
0 \rightarrow \mathrm{Gr}_{2}^{W} H^{1}(\mathfrak{n}) \rightarrow\left(\mathrm{Gr}_{-2}^{W} \mathfrak{n}\right)^{*} \stackrel{\text { bracket. }}{\longrightarrow} \Lambda^{2} \mathrm{Gr}_{1}^{W} H^{1}(\mathfrak{n}) \stackrel{\text { cup }}{\longrightarrow} \mathrm{Gr}_{2}^{W} H^{2}(\mathfrak{n}) \rightarrow 0 .
$$

6.4. Weighted completion of a profinite group. The weighted completion of a profinite group $\Gamma$ is a variant of a continuous relative completion. It is a useful tool as it can be used to define weight filtrations with strong exactness properties on the representations of $\Gamma$ that factor through its weighted completion.

Suppose that $\Gamma$ is a profinite group, $R$ is a reductive $\mathbb{Q}_{\ell}$-group endowed with a non-trivial, central cocharacter $\omega: \mathbb{G}_{m / \mathbb{Q}_{\ell}} \rightarrow R$, and that $\rho: \Gamma \rightarrow R\left(\mathbb{Q}_{\ell}\right)$ is a continuous, Zariski dense representation.

The weighted completion of $\Gamma$ with respect to $\rho$ is a proalgebraic $\mathbb{Q}_{\ell}$-group $\mathcal{G}$ that is a negatively weighted extension

$$
1 \rightarrow \mathcal{U} \rightarrow \mathcal{G} \rightarrow R \rightarrow 1
$$

of $R$ by a prounipotent $\mathbb{Q}_{\ell}$-group $\mathcal{U}$ and a continuous, Zariski dense representation $\tilde{\rho}: \Gamma \rightarrow \mathcal{G}\left(\mathbb{Q}_{\ell}\right)$ whose composition with $\mathcal{G}\left(\mathbb{Q}_{\ell}\right) \rightarrow R\left(\mathbb{Q}_{\ell}\right)$ is $\rho$. It is universal with respect to continuous homomorphisms of $\Gamma$ to negatively weighted extensions of $R$ that lift $\rho$.

Proposition 6.4 implies that every object of the category of finite dimensional $\mathcal{G}$-modules has a natural weight filtration and that the functor $\mathrm{Gr}_{\bullet}^{W}$ to the category of graded vector spaces is exact.

6.5. Presentations. Suppose that $F$ is a field of characteristic zero and that $R$ is a reductive $F$-group. A generalization of Levi's Theorem ensures that every extension

$$
1 \rightarrow \mathcal{U} \rightarrow \mathcal{G} \rightarrow R \rightarrow 1
$$

of $R$ by a prounipotent group splits and that any two such splittings are conjugate by an element of $\mathcal{U}$. Such a splitting induces an isomorphism

$$
\mathcal{G} \cong R \ltimes \mathcal{U}
$$

that commutes with the projections to $R$, where the action of $R$ on $\mathcal{U}$ is determined by the splitting. The prounipotent radical $\mathcal{U}$ with its $R$-action is determined by its Lie algebra $\mathfrak{u}$ and the action of $R$ on it. Thus, to give a presentation of $\mathcal{G}$, it suffices to give a presentation of $\mathfrak{u}$ in the category of $R$-modules.

By standard arguments (cf. [16, §3.1]), $\mathfrak{u}$ has a minimal presentation of the form

$$
\mathfrak{u} \cong \mathbb{L}\left(H_{1}(\mathfrak{u})\right)^{\wedge} /(\operatorname{im} \psi)
$$


in the category of $R$-modules, where $\mathbb{L}(V)$ denotes the free Lie algebra generated by the vector space $V$, and where $\psi: H_{2}(\mathfrak{u}) \rightarrow\left[\mathbb{L}\left(H_{1}(\mathfrak{u})\right)^{\wedge}, \mathbb{L}\left(H_{1}(\mathfrak{u})\right)^{\wedge}\right]$ is an injection such that the composite

$$
H_{2}(\mathfrak{u}) \rightarrow\left[\mathbb{L}\left(H_{1}(\mathfrak{u})\right)^{\wedge}, \mathbb{L}\left(H_{1}(\mathfrak{u})\right)^{\wedge}\right] \rightarrow \Lambda^{2} H_{1}(\mathfrak{u})
$$

is dual to the cup product $\Lambda^{2} H^{1}(\mathfrak{u}) \rightarrow H^{2}(\mathfrak{u})$.

Suppose now that $R$ is a reductive $\mathbb{Q}_{\ell}$-group and that $\omega: \mathbb{G}_{m} \rightarrow R$ is a central cocharacter. Suppose that $\Gamma$ is a profinite group and that $\rho: \Gamma \rightarrow R\left(\mathbb{Q}_{\ell}\right)$ is a continuous, Zariski dense representation. Denote the Lie algebra of the weighted completion of $\Gamma$ with respect to $\omega$ and $\rho$ by $\mathfrak{g}$ and that of its prounipotent radical by $\mathfrak{u}$.

Proposition 6.8 ([20, §4.3]). If $V$ is a finite dimensional $R$-module of weight $r$, then there are natural isomorphisms

$$
\operatorname{Hom}_{R}\left(H_{1}(\mathfrak{u}), V\right) \cong \operatorname{Hom}_{R}\left(\operatorname{Gr}_{r}^{W} H_{1}(\mathfrak{u}), V\right) \cong \begin{cases}H^{1}(\Gamma, V), & r<0, \\ 0, & r \geq 0\end{cases}
$$

and a natural injection $\operatorname{Hom}_{R}\left(H_{2}(\mathfrak{u}), V\right) \hookrightarrow H^{2}(\Gamma, V)$ whenever $r<0$. If $r>-2$, then $\operatorname{Hom}_{R}\left(H_{2}(\mathfrak{u}), V\right)=0$.

Remark 6.9. An elementary spectral sequence argument implies that when $R$ is connected,

$$
H^{j}(\mathfrak{g}, V) \cong H^{0}\left(\mathfrak{r}, H^{j}(\mathfrak{u}) \otimes V\right) \cong \operatorname{Hom}_{R}\left(H_{j}(\mathfrak{u}), V\right)
$$

for all finite dimensional $R$-modules $V$.

The following example summarizes results from [20] and will be used later in the paper.

Example 6.10. Suppose that $k$ is a field for which the image of the $\ell$-adic cyclotomic character $\chi_{\ell}: G_{k} \rightarrow \mathbb{Z}_{\ell}^{\times}$is infinite or, equivalently, that $\chi_{\ell}: G_{k} \rightarrow \mathbb{G}_{m}\left(\mathbb{Q}_{\ell}\right)$ is Zariski dense. Define a central cocharacte1 $\omega: \mathbb{G}_{m} \rightarrow \mathbb{G}_{m}$ by $z \mapsto z^{-2}$. Denote the weighted completion of $G_{k}$ with respect to $\chi_{\ell}$ and $\omega$ by $\mathcal{A}_{k}$. It is a split extension

$$
1 \rightarrow \mathcal{K}_{k} \rightarrow \mathcal{A}_{k} \rightarrow \mathbb{G}_{m} \rightarrow 1
$$

of $\mathbb{Q}_{\ell}$-groups, where $\mathcal{K}_{k}$ is prounipotent. Denote the Lie algebras of $\mathcal{A}_{k}$ and $\mathcal{K}_{k}$ by $\mathfrak{a}_{k}$ and $\mathfrak{k}_{k}$, respectively. Proposition 6.8 implies that

$$
\operatorname{Gr}_{r}^{W} H_{1}\left(\mathfrak{k}_{k}\right)=\left\{\begin{array}{lc}
H^{1}\left(G_{k}, \mathbb{Q}_{\ell}(s)\right)^{*} \otimes \mathbb{Q}_{\ell}(s), & r=-2 s<0, \\
0, & \text { otherwise }
\end{array}\right.
$$

This implies that $\operatorname{Gr}_{-1}^{W} \mathfrak{a}_{k}=0$ and that

$$
\mathrm{Gr}_{-2}^{W} \mathfrak{a}_{k} \cong H^{1}\left(G_{k}, \mathbb{Q}_{\ell}(1)\right)^{*} \otimes \mathbb{Q}_{\ell}(1) .
$$

If $k$ is a number field, a finite extension of $\mathbb{Q}_{p}$ or a finite field of characteristic $p$, where $p \neq \ell$, then the image of the $\ell$-adic cyclotomic character $\chi_{\ell}: G_{k} \rightarrow \mathbb{Z}_{\ell}^{\times}$is infinite. When $k$ is finite, $\mathcal{A}_{k}=\mathbb{G}_{m}$. When $k$ is a number field, $\mathrm{Gr}_{\bullet}^{W} \mathfrak{k}_{\mathfrak{k}}$ is a free Lie algebra, 20].

\footnotetext{
${ }^{5}$ This is the "right choice" because the standard representation of $\mathbb{G}_{m}$ corresponds to $\mathbb{Q}_{\ell}(1)$, which has weight -2 .
} 
6.6. An exactness criterion. In general, relative and weighted completion are only right exact. In this section we give a criterion for when weighted completion is exact. Suppose that

$$
1 \longrightarrow \pi \longrightarrow \widehat{\Gamma} \stackrel{\phi}{\longrightarrow} \Gamma \longrightarrow 1
$$

is an exact sequence of profinite groups. Suppose that $R$ is a reductive $\mathbb{Q}_{\ell}$-group with a central cocharacter $\omega: \mathbb{G}_{m} \rightarrow R$ and that $\rho: \Gamma \rightarrow R\left(\mathbb{Q}_{\ell}\right)$ is a continuous, Zariski dense representation. Denote the weighted completion of $\Gamma$ and $\widehat{\Gamma}$ with respect to $\rho$ and $\rho \circ \phi$ by $\mathcal{G}$ and $\widehat{\mathcal{G}}$, respectively. Denote the unipotent completion of $\pi$ by $\mathcal{P}$.

Proposition 6.11. Suppose that the $\Gamma$-action on $H_{1}(\pi)$ induces a $\mathcal{G}$-action on $H_{1}(\mathcal{P})$ and that the corresponding weight filtration on $H_{1}(\mathcal{P})$ has finite dimensional graded quotients which vanish in weights $r \geq 0$. If $\mathcal{P}$ has trivial center, then the sequence of completions

$$
1 \longrightarrow \mathcal{P} \longrightarrow \widehat{\mathcal{G}} \longrightarrow \mathcal{G} \longrightarrow 1
$$

is exact.

Proof. Denote the Lie algebra of $\mathcal{P}$ by $\mathfrak{p}$. Since the action of $\mathcal{G}$ on $H_{1}(\mathcal{P})$ is induced by the conjugation action of $\widehat{\Gamma}$ on $\pi$, it follows that the conjugation action of $\widehat{\Gamma}$ on $\pi$ induces an action of $\widehat{\mathcal{G}}$ on $H_{1}(\mathcal{P})=H_{1}(\mathfrak{p})$. Since this action is negatively weighted, it follows that the action of $\widehat{\Gamma}$ on $\mathfrak{p}$ induced by conjugation induces a negatively weighted action of $\widehat{\mathcal{G}}$ on $\mathfrak{p}$.

Right exactness of weighted completion implies that the sequence

$$
\mathcal{P} \rightarrow \widehat{\mathcal{G}} \rightarrow \mathcal{G} \rightarrow 1
$$

is exact. Since $\mathfrak{p}$ is negatively weighted and since each weight graded quotient of $H_{1}(\mathfrak{p})$ is finite dimensional, each $\mathfrak{p} / W_{r} \mathfrak{p}$ is finite dimensional and the group Aut $_{W} \mathfrak{p}$ of $W$ • preserving automorphisms of $\mathfrak{p}$ is proalgebraic as it is the inverse limit of $\operatorname{Aut}_{W}\left(\mathfrak{p} / W_{r}\right)$. The conjugation action of $\widehat{\Gamma}$ on $\pi$ induces a homomorphism $\widehat{\mathcal{G}} \rightarrow$ Aut $_{W} \mathfrak{p}$ such that the diagram

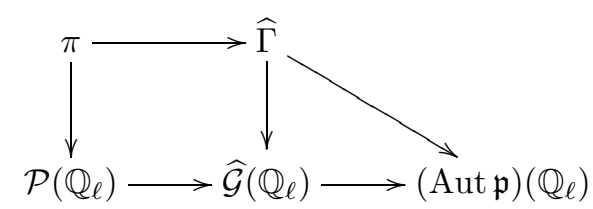

commutes. Since $\mathcal{P}$ has trivial center, the adjoint action $\mathcal{P} \rightarrow$ Aut $\mathfrak{p}$ is injective, which implies that $\mathcal{P} \rightarrow \widehat{\mathcal{G}}$ is injective.

\section{Weighted COMPLETiOn AND FAMilies OF CURVES}

Suppose that $k$ is a field of characteristic zero and that $C \rightarrow T$ is a family of smooth projective curves of genus $g \geq 2$ over a smooth, geometrically connected $k$-scheme $T$, or the generic point of a such a $k$-scheme. Let $\bar{\eta}_{T}$ be a geometric point of $T$ and $\bar{\eta}_{C}$ a geometric point of $C$ that lies over $\bar{\eta}_{T}$. Denote the fiber of $C$ over $\bar{\eta}_{T}$ by $C_{\bar{\eta}}$. Fix a prime number $\ell$. Set

$$
H=H_{\mathbb{Q}_{\ell}}=H_{\text {ét }}^{1}\left(C_{\bar{\eta}}, \mathbb{Q}_{\ell}(1)\right)
$$


and let $R$ be the Zariski closure of the image of the monodromy representation

$$
\pi_{1}\left(T, \bar{\eta}_{T}\right) \rightarrow \operatorname{GSp}\left(H_{\mathbb{Q}_{\ell}}\right) .
$$

Assume that $R$ contains the homotheties 6 Then one has the central cocharacter

$$
\omega: \mathbb{G}_{m} \rightarrow R, \quad z \mapsto z^{-1} \mathrm{id}_{H},
$$

which we shall call the standard cocharacter 7

The surjectivity of $\pi_{1}\left(C, \bar{\eta}_{C}\right) \rightarrow \pi_{1}\left(T, \bar{\eta}_{T}\right)$ implies that the monodromy representation $\pi_{1}\left(C, \bar{\eta}_{C}\right) \rightarrow R\left(\mathbb{Q}_{\ell}\right)$ is also Zariski dense. Denote the weighted completions of $\pi_{1}\left(C, \bar{\eta}_{C}\right)$ and $\pi_{1}\left(T, \bar{\eta}_{T}\right)$ with respect to $\omega$ and their monodromy representations to $R$ by $\mathcal{G}_{C}$ and $\mathcal{G}_{T}$, respectively; denote their prounipotent radicals by $\mathcal{U}_{C}$ and $\mathcal{U}_{T}$, respectively.

The surjectivity of $\pi_{1}\left(C \otimes_{k} \bar{k}, \bar{\eta}_{C}\right) \rightarrow \pi_{1}\left(T \otimes_{k} \bar{k}, \bar{\eta}_{T}\right)$ implies that their images in $R\left(\mathbb{Q}_{\ell}\right)$ are equal. Their common Zariski closure is a reductive subgroup $R^{\text {geom }}$ of $R$. Denote the continuous relative completions of $\pi_{1}\left(C \otimes_{k} \bar{k}, \bar{\eta}_{C}\right)$ and $\pi_{1}\left(T \otimes_{k} \bar{k}, \bar{\eta}_{T}\right)$ with respect to their homomorphisms to $R^{\text {geom }}\left(\mathbb{Q}_{\ell}\right)$ by $\mathcal{G}_{C}^{\text {geom }}$ and $\mathcal{G}_{T}^{\text {geom }}$, and their prounipotent radicals by $\mathcal{U}_{C}^{\text {geom }}$ and $\mathcal{U}_{T}^{\text {geom }}$.

Finally, set $\pi=\pi_{1}\left(C_{\bar{\eta}}, \bar{\eta}_{C}\right)$. Denote by $\pi^{\text {un }}$ its continuous unipotent completion $\pi_{/ \mathbb{Q}_{\ell}}^{\mathrm{un}}$ over $\mathbb{Q}_{\ell}$.

Proposition 7.1. With the notation above:

(i) There are exact sequences

$$
1 \rightarrow \pi^{\mathrm{un}} \rightarrow \mathcal{G}_{C} \rightarrow \mathcal{G}_{T} \rightarrow 1 \text { and } 1 \rightarrow \pi^{\mathrm{un}} \rightarrow \mathcal{G}_{C}^{\text {geom }} \rightarrow \mathcal{G}_{T}^{\text {geom }} \rightarrow 1
$$

of proalgebraic $\mathbb{Q}_{\ell}$-groups such that the diagram

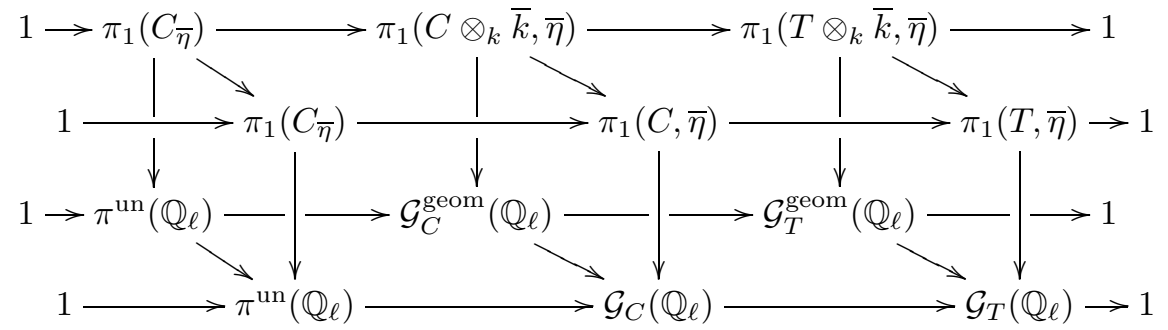

commutes.

(ii) Every section $s$ of $\pi_{1}(C, \bar{\eta}) \rightarrow \pi_{1}(T, \bar{\eta})$ induces a section $\sigma$ of $\mathcal{G}_{C} \rightarrow \mathcal{G}_{T}$ and a section $\sigma^{\text {geom }}$ of $\mathcal{G}_{C}^{\text {geom }} \rightarrow \mathcal{G}_{T}^{\text {geom }}$ such that the diagram

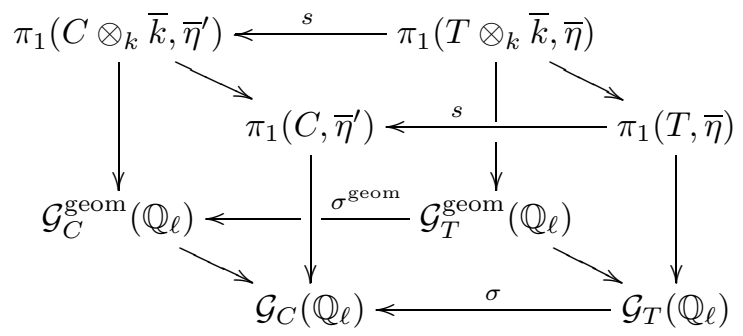

commutes.

\footnotetext{
${ }^{6}$ A theorem of Bogomolov $[3$ implies that this is always the case when $k$ is a number field.

${ }^{7}$ This is the "correct" definition as $H$ has weight -1 .
} 
Sketch of proof. The exactness of the row containing $\mathcal{G}_{C}$ follows from Proposition 6.11 and the fact 34 that $\pi^{\mathrm{un}}$ has trivial center as $H_{1}\left(\pi^{\mathrm{un}}\right)$ is a $\mathcal{G}_{C}$-module of weight -1 . The exactness of the row containing $\mathcal{G}_{C}^{\text {geom }}$ follows from the right exactness of relative completion and the fact that the composite $\pi^{\text {un }} \rightarrow \mathcal{G}_{C}^{\text {geom }} \rightarrow \mathcal{G}_{C}$ is injective.

Since $\mathcal{G}_{C}$ is a negatively weighted extension of $R$, the composition $\pi_{1}(T, \bar{\eta}) \rightarrow$ $\pi_{1}(C, \bar{\eta}) \rightarrow \mathcal{G}_{C}\left(\mathbb{Q}_{\ell}\right)$ of $\hat{\rho}_{C}$ with $s$ induces a homomorphism $\sigma: \mathcal{G}_{T} \rightarrow \mathcal{G}_{C}$. The universal mapping property of $\mathcal{G}_{T}$ implies that it is a section.

Denote the Lie algebras of

$$
R, \mathcal{G}_{C}, \mathcal{G}_{T}, \mathcal{U}_{C}, \mathcal{U}_{T}, \pi^{\text {un }} \quad \text { by } \mathfrak{r}, \mathfrak{g}_{C}, \mathfrak{g}_{T}, \mathfrak{u}_{C}, \mathfrak{u}_{T}, \mathfrak{p},
$$

respectively. All are representations of $\mathcal{G}_{C}$. So, by Proposition 6.6, these all have natural weight filtrations which are characterized by the property that their $m$ th graded quotient is an $R$-module of weight $m$. Moreover, $\mathfrak{p}=W_{-1} \mathfrak{p}$ and, for $X=T$ and $C$,

$$
\mathfrak{g}_{X}=W_{0} \mathfrak{g}_{X}, W_{-1} \mathfrak{g}_{X}=\mathfrak{u}_{X} \text { and } \operatorname{Gr}_{0}^{W} \mathfrak{g}_{X} \cong \mathfrak{r} .
$$

Corollary 7.2. There is an exact sequence

$$
0 \rightarrow \mathrm{Gr}_{\bullet}^{W} \mathfrak{p} \rightarrow \mathrm{Gr}_{\bullet}^{W} \mathfrak{g}_{C} \rightarrow \mathrm{Gr}_{\bullet}^{W} \mathfrak{g}_{T} \rightarrow 0
$$

of graded Lie algebras in the category of $R$-modules. Each section of $\pi_{1}\left(C, \bar{\eta}_{C}\right) \rightarrow$ $\pi_{1}\left(T, \bar{\eta}_{T}\right)$ induces an R-linear section of $\mathrm{Gr}_{\bullet}^{W} \mathfrak{g}_{C} \rightarrow \mathrm{Gr}_{\bullet}^{W} \mathfrak{g}_{T}$.

Proof. Proposition 7.1 implies that the sequence

$$
0 \rightarrow \mathfrak{p} \rightarrow \mathfrak{g}_{C} \rightarrow \mathfrak{g}_{T} \rightarrow 0
$$

of Lie algebras is an exact sequence of $\mathcal{G}_{C}$-modules. The result follows from the fact that the functor $\mathrm{Gr}_{\bullet}^{W}$ is exact on the category of $\mathcal{G}_{C}$-modules. The statement about sections follows similarly.

7.1. Weighted completion of Galois groups of function fields. Suppose that $T$ is a smooth, geometrically connected variety over a field $k$ and that $C \rightarrow T$ is a family of smooth projective curves over $T$. Set $K=k(T)$. Let $\bar{\eta}$ be a geometric point of $C$ that lies over the generic point of $T$.

Denote the restriction of $C$ to the Zariski open subset $U$ of $T$ by $C_{U}$. Denote the restriction of $C$ to the generic point Spec $K$ of $T$ by $C_{K}$. The image of

$$
\rho_{U}: \pi_{1}\left(C_{U}, \bar{\eta}\right) \rightarrow \pi_{1}(U, \bar{\eta}) \rightarrow \operatorname{GSp}\left(H_{\mathbb{Q}_{\ell}}\right)
$$

does not depend on $U$. Denote its Zariski closure by $R$. Assume that this contains the scalar matrices so that the image of the standard cocharacter (4), $\omega: z \mapsto$ $z^{-1} \mathrm{id}_{H}$, is defined. Denote the weighted completion of $\pi_{1}\left(C_{U}, \bar{\eta}\right)$ with respect to $\rho_{U}: \pi_{1}\left(C_{U}, \bar{\eta}\right) \rightarrow R\left(\mathbb{Q}_{\ell}\right)$ by $\mathcal{G}_{C / U}$. Denote the weighted completion of $\pi_{1}(U, \bar{\eta})$ by $\mathcal{G}_{U}$. Then one has the morphism of inverse systems

$$
\left\{\mathcal{G}_{C / U}\right\}_{U} \rightarrow\left\{\mathcal{G}_{U}\right\}_{U} .
$$

Denote the weighted completion of $\pi_{1}\left(C_{K}, \bar{\eta}\right)$ with respect to $\rho_{K}: \pi_{1}\left(C_{K}, \bar{\eta}\right) \rightarrow$ $R\left(\mathbb{Q}_{\ell}\right)$ by $\mathcal{G}_{C / K}$, and the weighted completion of $G_{K}$ with respect to the monodromy representation $G_{K} \rightarrow \mathrm{GSp}(H)$ by $\mathcal{G}_{K}$. 
Proposition 7.3. There are natural isomorphisms

$$
\mathcal{G}_{C / K} \stackrel{\simeq}{\longrightarrow} \lim _{U} \mathcal{G}_{C / U} \text { and } \mathcal{G}_{K} \stackrel{\simeq}{\longrightarrow} \lim _{U} \mathcal{G}_{U}
$$

such that the diagram

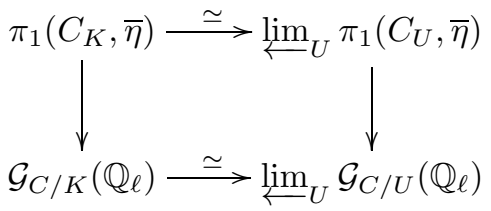

commutes. Each section of $\pi_{1}\left(C_{K}, \bar{\eta}\right) \rightarrow G_{K}$ induces a section of $\mathcal{G}_{C / K} \rightarrow \mathcal{G}_{K}$.

Proof. This follows from Proposition 6.8 and the fact that the natural homomorphisms

$$
\underset{U}{\lim _{U}} H^{\bullet}\left(\pi_{1}\left(C_{U}, \bar{\eta}\right), V\right) \rightarrow H^{\bullet}\left(\pi_{1}\left(C_{K}, \bar{\eta}\right), V\right), \underset{U}{\lim _{\vec{H}}} H^{\bullet}\left(\pi_{1}(U, \bar{\eta}), V\right) \rightarrow H^{\bullet}\left(G_{K}, V\right)
$$

are isomorphisms for all finite dimensional $R$-modules $V$. The statement about sections follows from the naturality of weighted completion.

\section{WeIGHTED COMPLETION OF ARITHMETIC MAPPING CLASS GROUPS}

In this section we summarize and extend results from 23. Throughout this section, $g, n$ and $m$ are non-negative integers satisfying $2 g-2+n>0$, and $m \geq 1$. We also assume that $k$ is a field of characteristic zero that contains $\boldsymbol{\mu}_{m}(\bar{k})$, the $m$ th roots of unity, and we fix a primitive $m$ th root of unity so that one has a geometrically connected moduli stack $\mathcal{M}_{g, n / k}[\mathrm{~m}]$.

Fix a geometric point $\bar{\eta}$ of $\mathcal{M}_{g, n / k}[m]$ and let $C_{\bar{\eta}}$ be the fiber of the universal curve over $\bar{\eta}$. For a prime number $\ell$ and a $\mathbb{Z}_{\ell \text {-module } A \text {, set }}$

$$
H_{A}=H_{\text {êt }}^{1}\left(C_{\bar{\eta}}, A(1)\right) \text {. }
$$

Assume that the image of the $\ell$-adic cyclotomic character $\chi_{\ell}: G_{k} \rightarrow \mathbb{Z}_{\ell}^{\times}$is infinite. This implies that the image of $\chi_{\ell}: G_{k} \rightarrow \mathbb{G}_{m}\left(\mathbb{Q}_{\ell}\right)$ is Zariski dense. Since the diagram

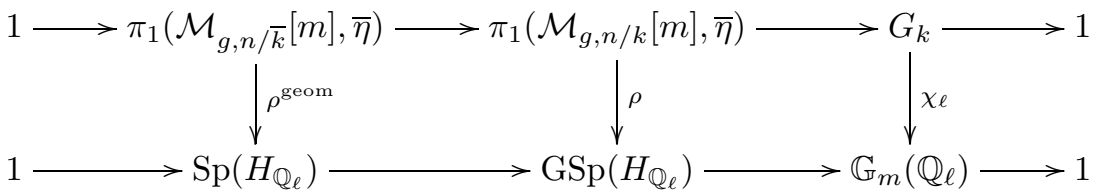

commutes and since the image of $\rho^{\text {geom }}$ is $\operatorname{Sp}\left(H_{\mathbb{Z}_{\ell}}\right)$, which is Zariski dense in $\operatorname{Sp}\left(H_{\mathbb{Q}_{\ell}}\right)$, the monodromy representation

$$
\rho: \pi_{1}\left(\mathcal{M}_{g, n / k}[m], \bar{\eta}\right) \rightarrow \operatorname{GSp}\left(H_{\mathbb{Q}_{\ell}}\right)
$$

is Zariski dense.

Now take the coefficient field $F$ to be $\mathbb{Q}_{\ell}$. Denote the weighted completion of $\pi_{1}\left(\mathcal{M}_{g, n / k}[m], \bar{\eta}\right)$ over $\mathbb{Q}_{\ell}$ with respect to $\rho$ and the standard cocharacter $\omega: z \mapsto$ $z^{-1} \mathrm{id}_{H}$ by

$$
\mathcal{G}_{\mathcal{M}_{g, n / k}[m]} \text { and } \tilde{\rho}: \pi_{1}\left(\mathcal{M}_{g, n / k}[m], \bar{\eta}\right) \rightarrow \mathcal{G}_{\mathcal{M}_{g, n / k}[m]}\left(\mathbb{Q}_{\ell}\right)
$$


Recall that $\mathcal{G}_{g, n}^{\text {geom }}$ is the relative completion of $\pi_{1}\left(\mathcal{M}_{g, n / \bar{k}}[m], \bar{\eta}\right)$ with respect to its standard representation to $\operatorname{Sp}\left(H_{\mathbb{Q}_{\ell}}\right)$.

Recall from Example 6.10 that $\mathcal{A}_{k}$ denotes the weighted completion of $G_{k}$ with respect to the $\ell$-adic cyclotomic character $\chi_{\ell}: G_{k} \rightarrow \mathbb{G}_{m}\left(\mathbb{Q}_{\ell}\right)$. The following is an easily proved consequence of the right exactness of relative and weighted completions.

Proposition 8.1 (23]). If $2 g-2+n>0$, then applying weighted completion to the two right-hand columns and relative completion to the left-hand column of the diagram

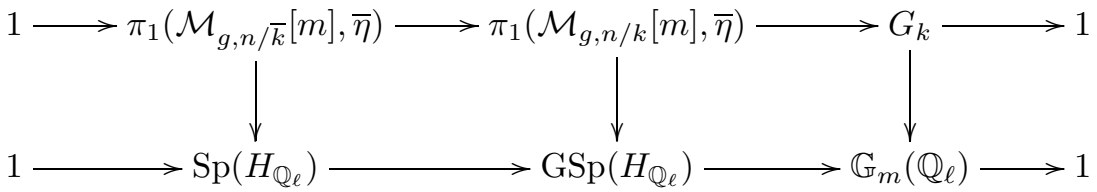

gives a commutative diagram

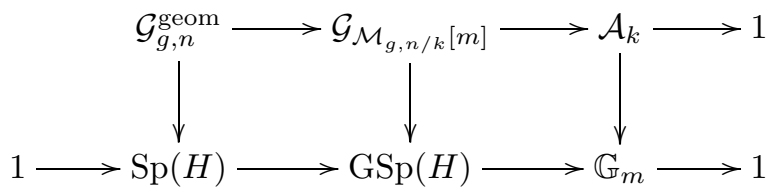

whose rows are exact.

Like the relative completion of mapping class groups, $\mathcal{G}_{\mathcal{M}_{g, n / k}[m]}$ does not depend on the level $m$ when $g \geq 3$.

Proposition 8.2. If $k$ is a field of characteristic zero and if $g \geq 3$, then for all $m \geq 1$ the natural homomorphism $\mathcal{G}_{\mathcal{M}_{g, n / k}[m]} \rightarrow \mathcal{G}_{\mathcal{M}_{g, n / k}}$ is an isomorphism.

Proof. This is a brief sketch. Complete details will appear in 23]. Set $\mathcal{M}=\mathcal{M}_{g, n}$. Denote the $\ell$-adic local system over $\mathcal{M}$ that corresponds to the $\operatorname{GSp}\left(H_{\mathbb{Q}_{\ell}}\right)$-module $V$ by $\mathbb{V}$. The spectral sequence

$$
H^{s}\left(G_{k}, H_{\text {êt }}^{t}\left(\mathcal{M}_{\bar{k}}, \mathbb{V}\right)\right) \Longrightarrow H_{\text {ét }}^{s+t}(\mathcal{M}, \mathbb{V})
$$

and the fact that the map $H_{\text {ét }}^{j}\left(\mathcal{M}_{/ \bar{k}}, \mathbb{V}\right) \rightarrow H_{\text {ét }}^{j}\left(\mathcal{M}_{/ \bar{k}}[m], \mathbb{V}\right)$ is an isomorphism when $j \leq 1$ and injective when $j=2$ implies that $H_{\text {ét }}^{j}(\mathcal{M}, \mathbb{V}) \rightarrow H_{\text {ét }}^{j}(\mathcal{M}[m], \mathbb{V})$ is also an isomorphism when $j \leq 1$ and injective when $j=2$. Proposition 6.8 implies that for all $\operatorname{GSp}(H)$-modules $V$ the columns of the commutative diagram

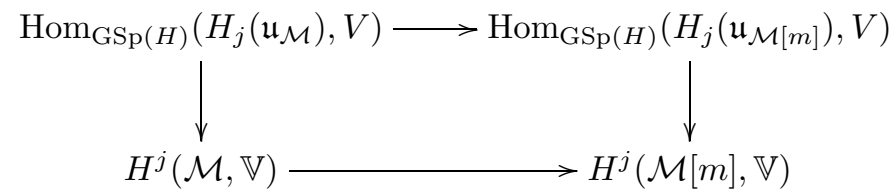

are isomorphisms when $j=1$ and injective when $j=2$. This implies that $H_{j}\left(\mathfrak{u}_{\mathcal{M}[m]}\right) \rightarrow H_{j}\left(\mathfrak{u}_{\mathcal{M}}\right)$ is an isomorphism when $j=1$ and surjective when $j=2$. The discussion of minimal presentations of pronilpotent Lie algebras in Section 6.5 implies that $\mathfrak{u}_{\mathcal{M}[m]} \rightarrow \mathfrak{u}_{\mathcal{M}}$ is an isomorphism 8

\footnotetext{
${ }^{8}$ The last step is the analogue of Stalling's criterion [40 for pronilpotent Lie algebras. It follows directly from the discussion of minimal presentations of pronilpotent Lie algebras in Section 6.5.
} 
Remark 8.3. The map $\mathcal{G}_{g, n}^{\text {geom }} \rightarrow \mathcal{G}_{\mathcal{M}_{g, n / k}}$ should be injective when $g \geq 3$. It is not injective when $g=1$. This issue is considered in [23] where it is related to the crystallinity of the action of $G_{k}$ on $\mathfrak{g}_{g, n}^{\text {geom }}$ when $g \geq 2$.

When the field $k$ is clear from the context, we will denote $\mathcal{G}_{\mathcal{M}_{g, n / k}}$ by $\mathcal{G}_{g, n}$ and its prounipotent radical by $\mathcal{U}_{g, n}$. Recall that $\mathcal{U}_{g, n}^{\text {geom }}$ denotes the prounipotent radical of $\mathcal{G}_{g, n}^{\text {geom }}$. Denote the Lie algebras of $\mathcal{U}_{g, n}$ and $\mathcal{U}_{g, n}^{\text {geom }}$ by $\mathfrak{u}_{g, n}$ and $\mathfrak{u}_{g, n}^{\text {geom }}$, respectively. Since $\mathfrak{u}_{g, n}$ is a $\mathcal{G}_{g, n}$-module (via the adjoint action) it has a natural weight filtration. Although it is not immediately obvious, $\mathfrak{u}_{g, n}^{\mathrm{geem}}$ also has a natural weight filtration.

Proposition $8.4([23])$. If $2 g-2+n>0$, then the natural action of $\pi_{1}\left(\mathcal{M}_{g, n / k}, \bar{\eta}\right)$ on $\pi_{1}\left(\mathcal{M}_{g, n / \bar{k}}, \bar{\eta}\right)$ induces an action of $\mathcal{G}_{g, n}$ on $\mathfrak{g}_{g, n}^{\text {geom }}$. That is, $\mathfrak{g}_{g, n}^{\text {geom }}$ and $\mathfrak{u}_{g, n}^{\text {geom }}$ are pro-objects of the category of $\mathcal{G}_{g, n}$-modules and thus have natural weight filtrations.

Remark 8.5. We will be appealing to computations in [15] where a weight filtration on $\mathfrak{g}_{g, n}^{\text {geom }}$ is constructed using Hodge theory. The weight filtration on $\mathfrak{g}_{g, n}^{\text {geom }}$ used in this paper is constructed using weighted completion. In order to apply the computations of [15] in this paper, we need to know that these two constructions of the weight filtration agree. General results imply that this is the case. However, in the present situation, a direct argument can be given when $g \geq 3$. The starting observation is that in both constructions $H_{1}(\mathfrak{p})$ has weight -1 . The exactness of $\mathrm{Gr}_{\bullet}^{W}$ then implies that $W_{-r} \mathfrak{p}$ is the $r$ th term of its lower central series, which implies that the two constructions of the weight filtration of $\mathfrak{p}$ agree. In both constructions, $\mathfrak{u}_{g, n}^{\text {geom }}=W_{-1} \mathfrak{g}_{g, n}^{\text {geom }}$ for all $n \geq 0$. Thus, to prove equality of the two constructions, it suffices to show that they agree on $\mathfrak{u}_{g, n}^{\text {geom }}$. When $g \geq 3$, Johnson's computation of the abelianization of the Torelli group [26] implies that the map $H_{1}\left(\mathfrak{u}_{g, 1}^{\text {geom }}\right) \rightarrow \operatorname{Gr}_{-1}^{W}$ Der $\mathfrak{p}$ is an isomorphism. This implies that $H_{1}\left(\mathfrak{u}_{g, 1}^{\text {geom }}\right)$ is pure of weight -1 in both constructions. The injectivity of the natural map

$$
H_{1}\left(\mathfrak{u}_{g, n}^{\text {geom }}\right) \rightarrow H_{1}\left(\mathfrak{u}_{g, 1}^{\text {geom }}\right)^{\oplus n}
$$

induced by $\mathcal{M}_{g, n} \rightarrow\left(\mathcal{M}_{g, 1}\right)^{n}$ when $n>1$, and the surjectivity of $H_{1}\left(\mathfrak{u}_{g, 1}^{\text {geom }}\right) \rightarrow$ $H_{1}\left(\mathfrak{u}_{g}^{\text {geom }}\right)$ imply that $H_{1}\left(\mathfrak{u}_{g, n}^{\text {geom }}\right)$ is also pure of weight -1 for all $n \geq 0$. The exactness of $\mathrm{Gr}_{\bullet}^{W}$ now implies that $W_{-r} \mathfrak{u}_{g, n}^{\text {geom }}$ is the $r$ th term of the lower central series of $\mathfrak{u}_{g, n}^{\text {geom }}$ for all $n \geq 0$. The two constructions therefore agree.

8.1. Variants. The constructions and results above extend to other moduli spaces. Here we consider one, namely the $n$th power of the universal curve $\mathcal{C}_{g / k}^{n}[m]$ over $\mathcal{M}_{g / k}[m]$. Others will be constructed in subsequent sections, as needed. As above, one has the homomorphism

$$
\rho: \pi_{1}\left(\mathcal{C}_{g / k}^{n}[m], \bar{\eta}\right) \rightarrow \operatorname{GSp}\left(H_{\mathbb{Q}_{\ell}}\right)
$$

which is Zariski dense. Denote the weighted completion of $\pi_{1}\left(\mathcal{C}_{g / k}^{n}[m], \bar{\eta}\right)$ with respect to $\rho$ and $\omega$ by $\mathcal{G}_{\mathcal{C}_{g / k}^{n}}[m]$. As in the case of $\mathcal{M}_{g, n / k}[m]$, it does not depend on $m$ when $g \geq 3$. We will henceforth denote it by $\widehat{\mathcal{G}}_{g, n}$ and its prounipotent radical by $\widehat{\mathcal{U}}_{g, n}$. Denote their Lie algebras by $\hat{\mathfrak{g}}_{g, n}$ and $\hat{\mathfrak{u}}_{g, n}$, respectively.

We will denote the completion of $\pi_{1}\left(\mathcal{C}_{g / \bar{k}}^{n}[m], \bar{\eta}\right)$ with respect to the natural homomorphism to $\operatorname{Sp}\left(H_{\mathbb{Q}_{\ell}}\right)$ by $\widehat{\mathcal{G}}_{g, n}^{\text {geom }}$. It too is independent of $m$ when $g \geq 3$. 
The inclusion $\mathcal{M}_{g, n / k}[m] \rightarrow \mathcal{C}_{g / k}^{n}[m]$ induces a homomorphism $\mathcal{G}_{g, n} \rightarrow \widehat{\mathcal{G}}_{g, n}$, and one has the commutative diagram

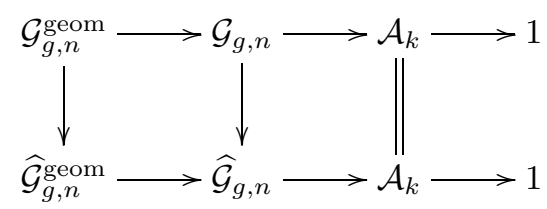

with exact rows.

8.2. Basepoints. Observe that $\mathcal{M}_{g, n / k}$ is a Zariski open subset of $\mathcal{C}_{g / k}^{n}$. Denote their common generic point by $\eta$ and let $\bar{\eta}$ be a geometric point that lies over it. Denote the image of $\bar{\eta}$ in $\mathcal{M}_{g / k}$ by $\bar{\eta}_{o}$ and the image of $\bar{\eta}$ in $\mathcal{M}_{g, 1 / k}$ under the $j$ th projection $\mathcal{M}_{g, n / k} \rightarrow \mathcal{M}_{g, 1 / k}$ by $\bar{x}_{j}$.

Denote the fiber of the universal curve over $\bar{\eta}_{o}$ by $C$. It has the $n$ rational points $\bar{x}_{1}, \ldots, \bar{x}_{n}$. The fiber of the projection $\mathcal{C}_{g / k}^{n} \rightarrow \mathcal{M}_{g / k}$ over $\bar{\eta}_{o}$ is $C^{n}$ and has basepoint $\mathbf{x}_{\bar{\eta}}:=\left(\bar{x}_{1}, \ldots, \bar{x}_{n}\right) \in C^{n}$. The natural map $\mathcal{C}_{g / k}^{n} \rightarrow\left(\mathcal{M}_{g, 1 / k}\right)^{n}$ takes the basepoint $\bar{\eta}$ of $\mathcal{C}_{g / k}^{n}$ to the basepoint $\mathbf{x}_{\bar{\eta}}$ of $\left(\mathcal{M}_{g, 1 / k}\right)^{n}$.

8.3. Exactness. The "fat diagonal" $\Delta$ of $C^{n}$ is the union of the divisors $\Delta_{i j}$, where

$$
\Delta_{i j}:=\left\{\left(u_{1}, \ldots, u_{n}\right): u_{i}=u_{j}, i \neq j\right\} .
$$

Observe that $\bar{x}_{\eta} \in C^{n}-\Delta$. The maps $\mathcal{M}_{g, n / k} \rightarrow \mathcal{C}_{g / k}^{n} \rightarrow\left(\mathcal{M}_{g, 1 / k}\right)^{n}$ induce a commutative diagram

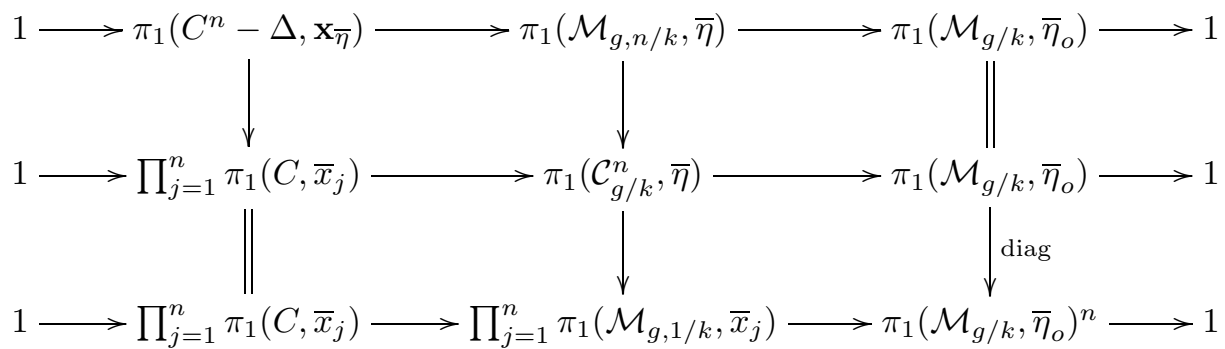

with exact rows 9 Denote $\pi_{1}\left(C^{n}-\Delta, \mathbf{x}_{\bar{\eta}}\right)$ by $\pi_{g, n}$ and the Lie algebra of its unipotent completion over $\mathbb{Q}_{\ell}$ by $\mathfrak{p}_{g, n}$. Denote the Lie algebra of $\pi_{1}\left(C, \bar{x}_{j}\right)^{\text {un }}$ by $\mathfrak{p}_{j}$ and the Lie algebra of $\pi_{1}\left(\mathcal{M}_{g, 1 / k}, \bar{x}_{j}\right)$ by $\mathfrak{g}_{g, 1}^{(j)}$.

Observe that the weight graded quotients of $\mathfrak{g}_{g, n}$ and $\hat{\mathfrak{g}}_{g, n}$ are $S_{n} \times \operatorname{GSp}(H)$ modules, where the $S_{n}$-action is induced by the action of $S_{n}$ on $\mathcal{C}_{g / k}^{n}$ that permutes the $n$ points.

\footnotetext{
${ }^{9}$ The exactness of both rows follows from the fact that the center of $\pi_{1}\left(C^{n}-\Delta, \mathbf{x}_{\bar{\eta}}\right)$ is trivial for all $n \geq 1$ when $g \geq 2$. The argument in the profinite case uses [1 and is essentially identical with the argument in the discrete case, which can be found in 2, Lem. 4.2.2].
} 
Proposition 8.6. Suppose that $g \geq 2$. After applying weighted completion to this diagram, we obtain the commutative diagram

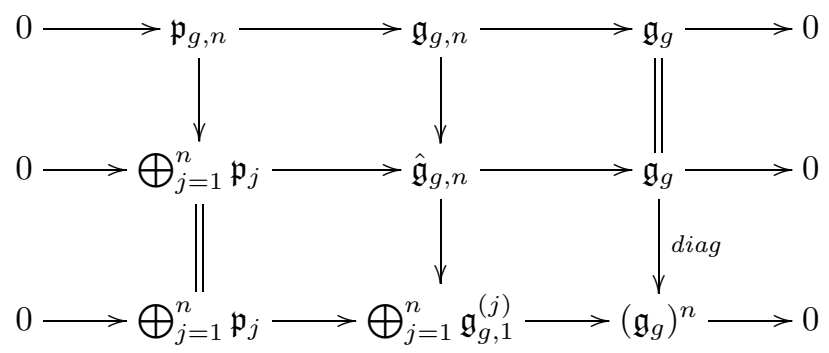

with exact rows. After applying $\mathrm{Gr}_{\bullet}^{W}$, the rows are exact sequences of $S_{n} \times \operatorname{GSp}(H)$ modules. Moreover, the same statement holds with $\mathfrak{g}$ replaced by $\mathfrak{g}^{\text {geom }}$ in the diagram.

Sketch of Proof. The conjugation action of $\pi_{1}\left(\mathcal{M}_{g, n / k}, \bar{\eta}\right)$ on $\pi_{1}\left(C^{n}-\Delta, \mathbf{x}_{\bar{\eta}}\right)$ induces an action of $\mathcal{G}_{g, n}$ on $H_{1}\left(\mathfrak{p}_{g, n}\right)$ which has weights -1 and -2 , and is therefore negatively weighted. The exactness of the first row now follows from Proposition 6.11 as $\mathfrak{p}_{g, n}$ has trivial center [34, p. 201]. The corresponding statement with $\mathfrak{g}$ replaced by $\mathfrak{g}^{\text {geom }}$ follows from the right exactness of relative completion and because the composite $\mathfrak{p}_{g, n} \rightarrow \hat{\mathfrak{g}}_{g, n}^{\text {geom }} \rightarrow \hat{\mathfrak{g}}_{g, n}$ is injective.

Right exactness of relative and weighted completion implies that the second row is right exact. To prove that it is exact, we use the map $r_{j}: \mathcal{C}_{g}^{n} \rightarrow \mathcal{M}_{g, 1}$ defined by $r_{j}:\left(x_{1}, \ldots, x_{n}\right) \rightarrow x_{j}$. It induces a commutative diagram

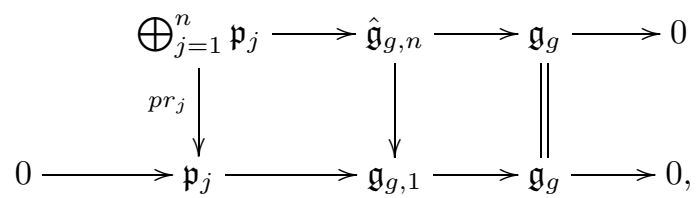

where $p r_{j}$ denotes projection onto the $j$ th factor. Proposition 7.1 implies that the bottom row of this diagram is exact. It follows that the top row of this diagram (i.e., the second row of the diagram in the statement) is left exact.

The exactness of the third row is a consequence of the exactness of the first row of the diagram in the case $n=1$.

Recall that for a fixed curve $C$, the $\operatorname{Gr}_{\bullet}^{W} \mathfrak{p}_{j}$ are naturally isomorphic. Denote their common value by $\mathrm{Gr}_{\bullet}^{W} \mathfrak{p}$.

The computations in [15, §12] imply directly that $\mathrm{Gr}_{-1}^{W} \mathfrak{p}_{g, n}$ is the $S_{n} \times \operatorname{GSp}(H)$ module $H^{n}$. They also imply that $\mathrm{Gr}_{-2}^{W} \mathfrak{p}_{g, n} \rightarrow\left(\mathrm{Gr}_{-2}^{W} \mathfrak{p}\right)^{n}$ is surjective with kernel isomorphic to $\bigoplus_{i<j} \mathbb{Q}_{\ell}(1)$, where the $i j$ th factor is generated by the logarithm 10 of a loop that encircles the $i j$ th diagonal $\Delta_{i j}$. Combined with the previous result, this gives:

Lemma 8.7. If $g \geq 3$, then

$$
\operatorname{Gr}_{r}^{W} \mathfrak{u}_{g, n} \rightarrow \operatorname{Gr}_{r}^{W} \hat{\mathfrak{u}}_{g, n} \text { and } \operatorname{Gr}_{r}^{W} \mathfrak{u}_{g, n}^{\text {geom }} \rightarrow \mathrm{Gr}_{r}^{W} \hat{\mathfrak{u}}_{g, n}^{\text {geom }}
$$

\footnotetext{
${ }^{10}$ Every element of a prounipotent group over a field of characteristic zero has a unique logarithm.
} 
are isomorphisms when $r=-1$ and are surjective when $r=-2$, both with kernel isomorphic to the $S_{n} \times \mathrm{GSp}(H)$-module $\bigoplus_{i<j} \mathbb{Q}_{\ell}(1)$, where $S_{n}$ acts by permuting the factors 11

Suppose that $n \geq 1$. Set $\pi:=\pi_{1}\left(C_{\bar{\eta}}, \bar{x}_{1}\right)$. Define $\mathfrak{p}$ to be the Lie algebra of $\pi^{\mathrm{un}}$. Then $\pi_{1}\left(\mathcal{M}_{g, n / k}[m], \bar{\eta}\right)$ acts on $\pi$ via the first projection $\pi_{1}\left(\mathcal{M}_{g, n / k}[m], \bar{\eta}\right) \rightarrow$ $\pi_{1}\left(\mathcal{M}_{g, 1 / k}[m], \bar{x}_{1}\right)$. This induces an action of $\mathcal{G}_{g, n}$ on $\mathfrak{p}$, so that $\mathfrak{p}$ also has a natural weight filtration. This action induces a Lie algebra homomorphism $\mathfrak{g}_{g, n} \rightarrow$ Der $\mathfrak{p}$ which preserves the weight filtration as it is $\mathcal{G}_{g, n}$-equivariant.

Proposition 8.8. If $g \geq 3$, the homomorphism $\mathfrak{u}_{g, 1} \rightarrow$ Der $\mathfrak{p}$ induces natural homomorphisms

$$
\mathrm{Gr}_{\bullet}^{W} \mathfrak{u}_{g, 1}^{\text {geom }} \rightarrow \mathrm{Gr}_{\bullet}^{W} \mathfrak{u}_{g, 1} \rightarrow \mathrm{Gr}_{\bullet}^{W} \operatorname{Der} \mathfrak{p} \cong \operatorname{Der~Gr}_{\bullet}^{W} \mathfrak{p}
$$

of Lie algebras in the category of $\operatorname{GSp}(H)$-modules. For all $n \geq 0$, the natural map

$$
\mathrm{Gr}_{-1}^{W} \mathfrak{u}_{g, n}^{\text {geom }} \rightarrow \mathrm{Gr}_{-1}^{W} \mathfrak{u}_{g, n}
$$

is an isomorphism. In weight -2 there is a short exact sequence

$$
0 \rightarrow \mathrm{Gr}_{-2}^{W} \mathfrak{u}_{g, n}^{\text {geom }} \rightarrow \mathrm{Gr}_{-2}^{W} \mathfrak{u}_{g, n} \rightarrow H^{1}\left(G_{k}, \mathbb{Q}_{\ell}(1)\right)^{*} \otimes \mathbb{Q}_{\ell}(1) \rightarrow 0 .
$$

Proof. Right exactness of weighted completion and the exactness of $\mathrm{Gr}_{m}^{W}$ imply that the sequence

$$
\mathrm{Gr}_{r}^{W} \mathfrak{u}_{g, n}^{\mathrm{geom}} \rightarrow \mathrm{Gr}_{r}^{W} \mathfrak{g}_{g, n} \rightarrow \mathrm{Gr}_{r}^{W} \mathfrak{a}_{k} \rightarrow 0
$$

is exact for all $r$. Recall from Example 6.10 that

$$
\operatorname{Gr}_{m}^{W} \mathfrak{a}_{k}= \begin{cases}0 & r=-1, \\ H^{1}\left(G_{k}, \mathbb{Q}_{\ell}(1)\right)^{*} \otimes \mathbb{Q}_{\ell}(1) & r=-2 .\end{cases}
$$

The map $\operatorname{Gr}_{r}^{W} \mathfrak{u}_{g, n}^{\text {geom }} \rightarrow \operatorname{Gr}_{r}^{W} \mathfrak{u}_{g, n}$ is therefore surjective when $r=-1$ and has cokernel $H^{1}\left(G_{k}, \mathbb{Q}_{\ell}(1)\right)^{*} \otimes \mathbb{Q}_{\ell}(1)$ when $r=-2$.

The computations in [15, Sections 9, 10] imply that the homomorphism

$$
\mathrm{Gr}_{r}^{W} \mathfrak{u}_{g, 1}^{\text {geom }} \rightarrow \operatorname{Der~Gr}_{r}^{W} \mathfrak{p}
$$

is an isomorphism when $r=-1$ and injective when $r=-2$. This completes the proof of the result when $n \leq 1$.

When $n>1$, Proposition 8.6 and the $n=1$ case imply that $\operatorname{Gr}_{r}^{W} \hat{\mathfrak{u}}_{g, n}^{\text {geom }} \rightarrow$ $\mathrm{Gr}_{r}^{W} \hat{\mathfrak{u}}_{g, n}$ is injective. The result now follows from Lemma 8.7.

\section{Generators and Relations}

The proofs of our main results make essential use of the structure of $\mathrm{Gr}_{\bullet}^{W} \mathfrak{u}_{g, n}^{\text {geom }} /$ $W_{-3}$ as a graded Lie algebra in the category of $S_{n} \times \mathrm{GSp}(H)$-modules. In this section we compute presentations of these Lie algebras. These results are refinements and elaborations of computations in [15, Section $11 \& 13]$.

\footnotetext{
${ }^{11}$ In case there is any confusion, the transposition $(i, j)$ acts trivially on the $i j$ th factor.
} 
9.1. Representations of $\operatorname{Sp}(H)$ and $\operatorname{GSp}(H)$. This is a quick review of the representation theory of $\operatorname{GSp}(H)$ over $\mathbb{Q}$, which is needed in subsequent sections. A good reference is the book [8] of Fulton and Harris. Throughout $H$ is a finite dimensional $\mathbb{Q}$-vector space which is endowed with a unimodular, skew-symmetric, $\operatorname{GSp}(H)$-invariant bilinear form $\theta: H \otimes H \rightarrow \mathbb{Q}(1)$. Since both groups are split over $\mathbb{Q}$, all of their irreducible representations are absolutely irreducible. The representations of $\operatorname{GSp}(H)$ and $\operatorname{Sp}(H)$ over a field $F$ of characteristic zero are obtained from their representations over $\mathbb{Q}$ by extension of scalars.

The standard cocharacter $\omega: z \mapsto z^{-1} \mathrm{id}_{H}$ assigns a weight $w(V)$ to each irreducible $\operatorname{GSp}(H)$-module $V$. The defining representation $H$ has weight -1 , which implies that $H^{\otimes r}$ has weight $-r$. As we recall below, the irreducible $\operatorname{GSp}(H)$ modules are constructed from $H^{\otimes r}$ using the symplectic form $\theta: H^{\otimes 2} \rightarrow \mathbb{Q}(1)$ and the action of the symmetric group $S_{r}$, which acts on $H^{\otimes r}$ by permuting the factors.

Recall from Section 4.1 that $\mathbb{Q}(r)$ denotes the 1-dimensional representation of $\operatorname{GSp}(H)$ with character $\tau^{r}: \operatorname{GSp}(H) \rightarrow \mathbb{G}_{m}$. Since the composite of the character $\tau: \operatorname{GSp}(H) \rightarrow \mathbb{G}_{m}$ with the standard cocharacter

$$
\mathbb{G}_{m} \stackrel{\omega}{\longrightarrow} \operatorname{GSp}(H) \stackrel{\tau}{\longrightarrow} \mathbb{G}_{m}
$$

is $x \mapsto x^{-2}, \mathbb{Q}(r)$ has weight $-2 r$. For any $\operatorname{GSp}(H)$-module $V$, set

$$
V(r)=V \otimes \mathbb{Q}(r) .
$$

We will call this a "Tate twist" of $V$. If $V$ has weight $w$, then $V(r)$ has weight $w-2 r$

For $1 \leq i<j \leq r$, define $\theta_{i j}: H^{\otimes r} \rightarrow H^{\otimes(r-2)}(1)$ to be the map

$$
\theta_{i j}: x_{1} \otimes \cdots \otimes x_{r} \mapsto \theta\left(x_{i}, x_{j}\right) x_{1} \otimes \cdots \otimes \widehat{x_{i}} \otimes \cdots \otimes \widehat{x_{j}} \otimes \cdots \otimes x_{n}
$$

that contracts the $i$ th and $j$ th factors using $\theta$. Set

$$
H^{\langle r\rangle}=\bigcap_{i<j} \operatorname{ker} \theta_{i j} .
$$

This is a $\operatorname{GSp}(H) \times S_{r}$-submodule of $H^{\otimes r}$.

The isomorphism classes of irreducible $S_{r}$-modules are parametrized by partitions

$$
\lambda=\left[\lambda_{1}, \lambda_{2}, \ldots, \lambda_{s}\right]
$$

of $r$ into $s \leq r$ parts, where $\lambda_{1} \geq \lambda_{2} \geq \cdots \geq \lambda_{s}>0$. Define $|\lambda|$ by

$$
|\lambda|:=r=\lambda_{1}+\lambda_{2}+\cdots+\lambda_{s} .
$$

To see how a partition $\lambda$ of $r$ gives a $\operatorname{GSp}(H)$-module, choose an irreducible $S_{r^{-}}$ module $W_{\lambda}$ that corresponds to the partition $\lambda$ and a non-zero element $w_{\lambda}$ of it. Set $S^{\lambda} H=\operatorname{Hom}_{S_{r}}\left(W_{\lambda}, H^{\otimes r}\right)$. This is a $\operatorname{GSp}(H)$-module, which we will identify with the image of the map $\operatorname{Hom}_{S_{r}}\left(W_{\lambda}, H^{\otimes r}\right) \rightarrow H^{\otimes r}$ that takes $\phi$ to $\phi\left(w_{\lambda}\right)$. Define

$$
H_{\lambda}=S^{\lambda} H \cap H^{\langle r\rangle} .
$$

This is a $\operatorname{GSp}(H)$-module of weight $-|\lambda|$. Note that $H_{\lambda}(r)$ has weight $-|\lambda|-2 r$.

Set $g=\operatorname{dim} H / 2$. The basic general facts we need from representation theory are summarized in the following result, which can be deduced from standard results, such as those presented in $[8]$. 
Theorem 9.1. The representations $H_{\lambda}(r)$, where $r \in \mathbb{Z}$ and $\lambda$ is a partition of a non-negative integer into $\leq g$ parts, form a set of representatives of the isomorphism classes of the irreducible $\operatorname{GSp}(H)$-modules. Each remains irreducible when restricted to $\mathrm{Sp}(H)$; every irreducible $\mathrm{Sp}(H)$-module arises in this way. Two irreducible $\operatorname{GSp}(H)$-modules $H_{\lambda}(r)$ and $H_{\lambda^{\prime}}\left(r^{\prime}\right)$ are isomorphic as $\operatorname{Sp}(H)$-modules if and only if $\lambda=\lambda^{\prime}$.

9.2. A presentation of $\mathrm{Gr}_{\bullet}^{W} \mathfrak{p}$. Here $\mathfrak{p}$ denotes the Lie algebra of the unipotent completion of the fundamental group of a smooth complex projective curve of genus $g \geq 1$. Its natural weight filtration is defined over $\mathbb{Q}$ as $W_{-r} \mathfrak{p}$ is the $r$ th term of its lower central series. We recall the presentation of $\operatorname{Gr}_{\bullet}^{W} \mathfrak{p}$. Recall that $\check{\theta}$ is a map $\mathbb{Q}(1) \rightarrow \Lambda^{2} H$. It will be regarded as a map $\check{\theta}: \mathbb{Q}(1) \rightarrow \mathbb{L}_{2}(H)$ via the canonical identification $\mathbb{L}_{2}(H) \cong \Lambda^{2} H$. The next result follows directly from a fundamental result of Labute [32. It also follows from Hodge theory via the main result of [5] or by the more motivic argument in [15, Thm. 5.8].

Proposition 9.2. If $g \geq 1$, then the weight filtration of $\mathfrak{p}$ equals its lower central series. There is a natural $\mathrm{GSp}(H)$-equivariant Lie algebra isomorphism

$$
\mathrm{Gr}_{\bullet}^{W} \mathfrak{p} \cong \mathbb{L}(H) /(\operatorname{im} \check{\theta}) .
$$

Inner automorphisms act trivially on this isomorphism, so that it does not depend on the choice of a basepoint.

A direct computation using the method of [15, §8] and the fact (cf. [30, (4.1)]) that $H^{\bullet}\left(\mathrm{Gr}_{\bullet}^{W} \mathfrak{p}\right) \cong H^{\bullet}(\pi, \mathbb{Q})$ yields:

Corollary 9.3. If $g \geq 1$ and $1 \leq r<6$, then $\left(\mathrm{Gr}_{-r}^{W} \mathfrak{p}\right)^{\mathrm{Sp}(H)}=0$.

Proof. When $g=1, \mathfrak{p}$ is abelian and the result is trivially true. Suppose that $g \geq 2$. The trivial $\operatorname{Sp}(H)$-module can occur in $\mathrm{Gr}_{-r}^{W} \mathfrak{p}$ only when $r$ is even. So we need to check that there are no copies of the trivial representation in $\operatorname{Gr}_{-2}^{W} \mathfrak{p}$ and $\mathrm{Gr}_{-4}^{W} \mathfrak{p}$. The proof of [15, Prop. 8.4] implies that for all $g \geq 2, \mathrm{Gr}_{-1}^{W} \mathfrak{p}=H$, $\operatorname{Gr}_{-2}^{W} \mathfrak{p}=\Lambda^{2} H / \operatorname{im} \check{\theta}$, and that $\operatorname{Gr}_{-3}^{W} \mathfrak{p}$ is the irreducible $\operatorname{Sp}(H)$-module that is the highest weight part of $H \otimes \Lambda^{2} H$, which is isomorphic to $H_{[2,1]}$. Each is an irreducible $\operatorname{Sp}(H)$-module. The case $r=2$ follows. When $r=4$, [15, Cor. 8.3] implies that

$$
\left[\mathrm{Gr}_{-4}^{W} \mathfrak{p}\right]=\left[\mathrm{Gr}_{-1}^{W} \mathfrak{p} \otimes \mathrm{Gr}_{-3}^{W} \mathfrak{p}\right]+\left[\Lambda^{2} \mathrm{Gr}_{-2}^{W} \mathfrak{p}\right]-\left[\mathrm{Gr}_{-2}^{W} \mathfrak{p} \otimes \Lambda^{2} \mathrm{Gr}_{-1}^{W} \mathfrak{p}\right]+\left[\Lambda^{4} \mathrm{Gr}_{-1}^{W} \mathfrak{p}\right]
$$

in the representation ring12 of $\operatorname{Sp}(H)$. Schur's Lemma implies that the first two terms do not contain a copy of the trivial representation and that the third term contains one. The decomposition

$$
\Lambda^{4} H \cong \begin{cases}H_{\left[1^{4}\right]} \oplus H_{\left[1^{2}\right]} \oplus \mathbb{Q} & g \geq 4, \\ H_{\left[1^{2}\right]} \oplus \mathbb{Q} & g=3, \\ \mathbb{Q} & g=2,\end{cases}
$$

into irreducible $\operatorname{Sp}(H)$ modules implies that the fourth term contains one copy of the trivial representation. The result follows.

The same method can be used to show that $\operatorname{dim}\left(\operatorname{Gr}_{-6}^{W} \mathfrak{p}\right)^{\operatorname{Sp}(H)}=1$ for all $g \geq 2$.

\footnotetext{
${ }^{12}$ The class of the $G$-module $V$ in the representation $\operatorname{ring}$ of $G$ is denoted by $[V]$.
} 
9.3. Frequently used $\operatorname{GSp}(H)$-modules. Several GSp $(H)$-modules play a significant role in this paper. All are of weight -1 or -2 and are Tate twists of the representations that correspond to the partitions $[1],\left[1^{2}\right]:=[1,1]$, and $\left[1^{3}\right]:=[1,1,1]$. We first define integral models of each as we will need them in the genus 3 case.

The form $\theta$ will be a unimodular, skew-symmetric bilinear form on $H_{\mathbb{Z}}$ and gives rise to a $\operatorname{GSp}(H)$-equivariant mapping $\Lambda^{2} H_{A} \rightarrow A(1)$ for all commutative rings $A$. The dual form $\tilde{\theta}$, defined in Section 4.1 will be regarded both as an equivariant map $\check{\theta}: A(1) \rightarrow \Lambda^{2} H_{A}$ and as an element of $\Lambda^{2} H_{A}(-1)$. Set

$$
\Lambda_{0}^{2} H_{A}:=\Lambda^{2} H_{A} / \operatorname{im} \check{\theta} \text { and } \Lambda_{0}^{3} H_{A}:=\left(\Lambda^{3} H_{A}\right)(-1) / \check{\theta} \wedge H_{A} .
$$

When $A$ is a field of characteristic zero, $\Lambda_{0}^{2} H_{A}$ and $\Lambda_{0}^{3} H_{A}$ are the irreducible GSp $(H)$-modules $H_{\left[1^{2}\right]}$ and $H_{\left[1^{3}\right]}(-1)$. These have weights -2 and -1 , respectively.

Lemma 9.4. If $A$ is an integral domain of characteristic zero, then the short exact sequence

$$
0 \longrightarrow A(1) \stackrel{\check{\theta}}{\longrightarrow} \Lambda^{2} H_{A} \longrightarrow \Lambda_{0}^{2} H_{A} \longrightarrow 0
$$

of $\operatorname{GSp}\left(H_{A}\right)$-modules splits if and only if $g \in A^{\times}$. The short exact sequence

$$
0 \longrightarrow H_{A} \stackrel{\check{\theta} \wedge}{\longrightarrow}\left(\Lambda^{3} H_{A}\right)(-1) \longrightarrow \Lambda_{0}^{3} H_{A} \longrightarrow 0
$$

of $\operatorname{GSp}\left(H_{A}\right)$-modules splits if and only if $g-1 \in A^{\times}$.

Proof. We will prove the lemma for the exact sequence (6). The proof for the exact sequence (5) is similar and is left to the reader. The map $c: \Lambda^{3} H_{A} \rightarrow H_{A}(1)$ defined by

$$
c: x \wedge y \wedge z \mapsto \theta(x, y) z+\theta(y, z) x+\theta(z, x) y
$$

is $\operatorname{GSp}\left(H_{A}\right)$-equivariant. Its composition $c \circ j$ with the inclusion $j: H_{A}(1) \hookrightarrow \Lambda^{3} H_{A}$ is $(g-1)$ times the identity of $H_{A}(1)$. If $g-1 \in A^{\times}$, then $c /(g-1)$ splits (6) twisted by $A(1)$.

Suppose now that $s: \Lambda^{3} H_{A}(-1) \rightarrow H_{A}$ is a splitting of ([6). Denote the fraction field of $A$ by $F$. Since $F$ has characteristic zero, $H_{F}$ and $\Lambda_{0}^{3} H_{F}$ are irreducible $\operatorname{GSp}\left(H_{F}\right)$-modules and the splitting of (6) $\otimes_{A} F$ is unique, and so must be $c /(g-1)$. This and the fact that $A$ is a domain imply that $s=c /(g-1)$. Since $\theta$ is unimodular, the image of $c /(g-1): \Lambda^{3} H_{A}(-1) \rightarrow H_{F}$ is contained in $H_{A}$ if and only if $g-1 \in$ $A^{\times}$.

The lemma implies that for all fields $F$ of characteristic zero,

$$
\Lambda^{2} H_{F} \cong A(1) \oplus \Lambda_{0}^{2} H_{F} \text { and }\left(\Lambda^{3} H_{F}\right)(-1) \cong H_{F} \oplus \Lambda_{0}^{3} H_{F} .
$$

We fix these isomorphisms to be the ones given by the unique $\operatorname{GSp}(H)$-invariant splittings of the sequences in the lemma above. From this point on, we will write $H$ instead of $H_{F}$ when $F$ is a field of characteristic zero. The default field of characteristic zero in this section will be $\mathbb{Q}$. In later sections it will be $\mathbb{Q}_{\ell}$.

Another representation that will occur, but which plays a minor role, is

$$
V_{\boxplus}:=H_{[2,2]}(-1),
$$

which has weight -2 . The representation $H_{[2,2]}$ is the highest weight part of the second symmetric power of $\Lambda_{0}^{2} H$. 
Kabanov's stability result 27] implies that when $g \geq 6$ the decomposition of $\Lambda^{2} \Lambda_{0}^{3} H$ is independent of $g$ in the sense described in [15, $\left.\S 6\right]$. Because of this, one can compute this decomposition for all $g \geq 6$ by computing it when $g=6$. The following computations were made using the computer program LiE.

Proposition 9.5 (cf. [15, Lem. 10.2]). If $g \geq 3$, then each irreducible $\mathrm{GSp}(H)$ module that occurs in $\Lambda^{2} \Lambda_{0}^{3} H$ occurs with multiplicity one. When $g=3$,

$$
\Lambda^{2}\left(\Lambda_{0}^{3} H\right)=\mathbb{Q}(1) \oplus V_{\boxplus} ;
$$

when $g \geq 4$,

$$
\Lambda^{2}\left(\Lambda_{0}^{3} H\right) \supset \mathbb{Q}(1) \oplus \Lambda_{0}^{2} H \oplus V_{\boxplus}
$$

Moreover, the representation $H \otimes \Lambda_{0}^{3} H$ contains a unique copy of $\Lambda_{0}^{2} H$ for all $g \geq 3$.

9.4. Presentation of $\mathrm{Gr}_{\bullet}^{W} \mathfrak{u}_{g, 1}^{\text {geom }} / W_{-3}$. The Lie algebra $\mathrm{Gr}_{\bullet}^{W} \mathfrak{u}_{g, 1}^{\text {geom }} / W_{-3}$ can be computed by considering its action on $\mathrm{Gr}_{\bullet}^{W} \mathfrak{p}$.

Our first task is to determine $\mathrm{Gr}_{\bullet}^{W} \operatorname{Der} \mathfrak{p} / W_{-3}$. Fix a field $F$ of characteristic zero. Denote the free Lie algebra generated by the $F$-vector space $V$ by $\mathbb{L}(V)$.

The free Lie algebra $\mathbb{L}(V)$ is graded by bracket length:

$$
\mathbb{L}(V) \cong \bigoplus_{n \geq 1} \mathbb{L}_{n}(V)
$$

Observe that the derivation Lie algebra $\operatorname{Der} \mathbb{L}(H)$ is isomorphic to $\operatorname{Hom}_{F}(H, \mathbb{L}(H))$ and is also graded:

$$
\operatorname{Der} \mathbb{L}(H)=\bigoplus_{n \geq 0} \operatorname{Der}_{n} \mathbb{L}(H),
$$

where $\operatorname{Der}_{n} \mathbb{L}(H):=\operatorname{Hom}\left(H, \mathbb{L}_{n+1}(H)\right)$. Observe that there are natural $\operatorname{GSp}(H)$ actions on $\mathbb{L}\left(\left(\Lambda^{3} H\right)(-1)\right)$ and $\operatorname{Der} \mathbb{L}(H)$. The following fact is well known, but we prove it because of its importance in this paper. First note if $x, y \in H$, then $\theta(x, y) \check{\theta} \in \Lambda^{2} H$.

Lemma 9.6. If $g \geq 2$, there is a natural $\operatorname{GSp}(H)$-equivariant, graded Lie algebra homomorphism

$$
\tilde{\delta}: \mathbb{L}\left(\left(\Lambda^{3} H\right)(-1)\right) \rightarrow \operatorname{Der} \mathbb{L}(H)
$$

such that

(i) $\tilde{\delta}(u)$ annihilates the image of $\check{\theta}$ for all $u \in \mathbb{L}\left(\left(\Lambda^{3} H\right)(-1)\right)$;

(ii) $\tilde{\delta}(x \wedge \check{\theta})=\operatorname{ad}_{x}-\theta(x, \quad) \check{\theta}$ for all $x \in H$.

Moreover

$$
\operatorname{im}\left\{\mathbb{L}_{2}\left(\left(\Lambda^{3} H\right)(-1)\right) \rightarrow \operatorname{Hom}\left(H, \mathbb{L}_{3}(H)\right)\right\} \cong V_{\boxplus} \oplus \Lambda_{0}^{2} H \oplus F(1) .
$$

Proof. It suffices to define a $\operatorname{GSp}(H)$-equivariant linear mapping

$$
\Lambda^{3} H \rightarrow\left[\operatorname{Der}_{1} \mathbb{L}(H)\right](1)=\operatorname{Hom}\left(H, \mathbb{L}_{2}(H)(1)\right) .
$$

This homomorphism is defined by

$$
\tilde{\delta}: x \wedge y \wedge z: u \mapsto \theta(u, x)[y, z]+\theta(u, y)[z, x]+\theta(u, z)[x, y]
$$

The identities are easily verified.

Since each element of $\mathbb{L}\left(\left(\Lambda^{3} H\right)(-1)\right)$ annihilates $\operatorname{im} \check{\theta} \in \mathbb{L}_{2}(H), \tilde{\delta}$ induces a $\operatorname{GSp}(H)$-equivariant graded Lie algebra homomorphism

$$
\delta: \mathbb{L}\left(\left(\Lambda^{3} H\right)(-1)\right) \rightarrow \operatorname{Der} \mathrm{Gr}_{\bullet}^{W} \mathfrak{p} .
$$


Theorem 9.7 ([15, Cor. 5.7 and $\S 11]$ ). If $g \geq 3$, then there is a Lie algebra surjection

such that the diagram

$$
q: \mathbb{L}\left(\left(\Lambda^{3} H\right)(-1)\right) \rightarrow \mathrm{Gr}_{\bullet}^{W} \mathfrak{u}_{g, 1}^{\text {geom }}
$$

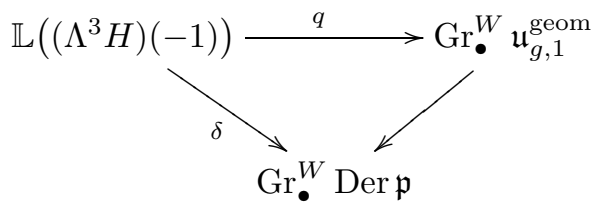

commutes. It induces isomorphisms

$$
\mathrm{Gr}_{r}^{W} \mathfrak{u}_{g, 1}^{\text {geom }} \stackrel{\simeq}{\longrightarrow} \operatorname{Gr}_{r}^{W} \operatorname{Der} \mathfrak{p} \cong \begin{cases}\left(\Lambda^{3} H\right)(-1) & r=-1, \\ V_{\boxplus} \oplus \Lambda_{0}^{2} H & r=-2 .\end{cases}
$$

The isomorphism $\operatorname{Gr}_{-1}^{W}$ Der $\mathfrak{p} \cong \Lambda^{3} H(-1)$ when $g \geq 3$ is a manifestation of the Johnson homomorphism [26]. It is induced by the homomorphism (7). The composition of $\delta$ with inclusion $i: H \hookrightarrow\left(\Lambda^{3} H\right)(-1)$ defined by $x \mapsto x \wedge \check{\theta}$ takes $x \in H$ to the inner derivation $\operatorname{ad}_{x}$ of $\mathfrak{p}$. Identify the copy of $\Lambda_{0}^{2} H$ in $\operatorname{Gr}_{-2}^{W}$ Der $\mathfrak{u}_{g, 1}^{\text {geom }}$ with the image of the mapping

$$
\Lambda^{2} H \stackrel{\Lambda^{2} i}{\longrightarrow} \Lambda^{2}\left(\left(\Lambda^{3} H\right)(-1)\right) \stackrel{\text { bracket }}{\longrightarrow} \mathrm{Gr}_{-2}^{W} \mathfrak{u}_{g, 1}^{\text {geom }}
$$

induced by the bracket.

Since the isomorphisms (8) factor through the map $\operatorname{Gr}_{r}^{W} \mathfrak{u}_{g, 1}^{\text {geom }} \rightarrow \operatorname{Gr}_{r}^{W} \mathfrak{u}_{g, 1}$, we conclude:

Corollary 9.8. If $g \geq 3$, then $\operatorname{Gr}_{r}^{W} \mathfrak{u}_{g, 1}^{\text {geom }} \rightarrow \operatorname{Gr}_{r}^{W} \mathfrak{u}_{g, 1}$ is injective when $r=-1,-2$.

We also need to understand the bracket

$$
\Lambda^{2} \mathrm{Gr}_{-1}^{W} \mathfrak{u}_{g, 1}^{\text {geom }} \rightarrow \mathrm{Gr}_{-2}^{W} \mathfrak{u}_{g, 1}^{\text {geom }} .
$$

With respect to the decomposition $\left(\Lambda^{3} H\right)(-1)=H \oplus \Lambda_{0}^{3} H$, the bracket has three components:

(10) $\quad \Lambda^{2} H \rightarrow \mathrm{Gr}_{-2}^{W}$ Der $\mathfrak{p}, \quad H \otimes \Lambda_{0}^{3} H \rightarrow \mathrm{Gr}_{-2}^{W}$ Der $\mathfrak{p}, \quad \Lambda^{2}\left(\Lambda_{0}^{3} H\right) \rightarrow \mathrm{Gr}_{-2}^{W}$ Der $\mathfrak{p}$.

Each is $\operatorname{GSp}(H)$-equivariant. These can be computed in Der $\operatorname{Gr}_{\bullet}^{W} \mathfrak{p}$.

Proposition 9.9 ([15, $\S 12])$. When $g \geq 3$, the images of the three components of the bracket (91) are:

$$
\begin{aligned}
\operatorname{im}\left\{\Lambda^{2} H \rightarrow \mathrm{Gr}_{-2}^{W} \operatorname{Der} \mathfrak{p}\right\} & =\Lambda_{0}^{2} H, \\
\operatorname{im}\left\{H \otimes \Lambda_{0}^{3} H \rightarrow \mathrm{Gr}_{-2}^{W} \operatorname{Der} \mathfrak{p}\right\} & =\Lambda_{0}^{2} H, \\
\operatorname{im}\left\{\Lambda^{2}\left(\Lambda_{0}^{3} H\right) \rightarrow \mathrm{Gr}_{-2}^{W} \operatorname{Der} \mathfrak{p}\right\} & = \begin{cases}V_{\boxplus} & g=3, \\
V_{\boxplus} \oplus \Lambda_{0}^{2} H & g \geq 4 .\end{cases}
\end{aligned}
$$

Remark 9.10. The genus 2 story is very different for several reasons, one of which is that every genus 2 curve is hyperelliptic. On the representation theoretic side, $\Lambda^{3} H \cong H(1)$; the image of the homomorphism $\mathbb{L}\left(\Lambda^{3} H(-1)\right) \rightarrow$ Der $\mathfrak{p}$ is the set of inner derivations and the image of $\mathrm{Gr}_{\bullet}^{W} \mathfrak{u}_{2,1} \rightarrow \mathrm{Gr}_{\bullet}^{W}$ Der $\mathfrak{p}$ is generated by this and by the copy of $V_{\boxplus}$ in $\mathrm{Gr}_{-2}^{W}$ Der $\mathfrak{p}$. 
9.5. Presentations of $\mathrm{Gr}_{\bullet}^{W} \hat{\mathfrak{u}}_{g, n}^{\text {geom }} / W_{-3}$ and $\mathrm{Gr}_{\bullet}^{W} \mathfrak{u}_{g, n}^{\text {geom }} / W_{-3}$. The computations of the previous section combined with the results of Section 8 yield computations of $\mathrm{Gr}_{\bullet}^{W} \hat{\mathfrak{u}}_{g, n}^{\text {geom }} / W_{-3}$ and $\mathrm{Gr}_{\bullet}^{W} \mathfrak{u}_{g, n}^{\text {geom }} / W_{-3}$.

Denote the image of $u \in \Lambda^{3} H$ in $\left(\Lambda_{0}^{3} H\right)(1)$ by $\bar{u}$. For a positive integer $n$, define

$$
\Lambda_{n}^{3} H=\left\{\left(u_{1}, \ldots, u_{n}\right) \in\left(\Lambda^{3} H\right)^{n}: \bar{u}_{1}=\cdots=\bar{u}_{n}\right\}(-1) \cong \Lambda_{0}^{3} H \oplus H^{\oplus n} .
$$

This has weight -1 . Note that $\Lambda_{1}^{3} H=\left(\Lambda^{3} H\right)(-1)$. The following result is proved later in the section.

Theorem 9.11. If $g \geq 3$, then for all $n \geq 0$ there are natural $S_{n} \times \operatorname{GSp}(H)$ equivariant isomorphisms

$$
H_{1}\left(\hat{\mathfrak{u}}_{g, n}^{\text {geom }}\right) \cong H_{1}\left(\mathfrak{u}_{g, n}^{\text {geom }}\right) \cong \operatorname{Gr}_{-1}^{W} \mathfrak{u}_{g, n}^{\text {geom }} \cong \Lambda_{n}^{3} H .
$$

There is an exact sequence

$$
0 \rightarrow \mathbb{Q}_{\ell}(1)^{\left(\begin{array}{c}
n \\
2
\end{array}\right)} \rightarrow \mathrm{Gr}_{-2}^{W} \mathfrak{u}_{g, n}^{\text {geom }} \rightarrow \mathrm{Gr}_{-2}^{W} \hat{\mathfrak{u}}_{g, n}^{\text {geom }} \rightarrow 0
$$

of $S_{n} \times \operatorname{GSp}(H)$-modules and an $S_{n} \times \operatorname{GSp}(H)$-equivariant isomorphism

$$
\mathrm{Gr}_{-2}^{W} \mathfrak{u}_{g, n}^{\text {geom }} \cong V_{\boxplus} \oplus\left(\Lambda_{0}^{2} H\right)^{n} \oplus \mathbb{Q}_{\ell}(1)^{\left(\begin{array}{c}
n \\
2
\end{array}\right)} .
$$

Our next task is to describe the bracket

$$
\text { bracket : } \Lambda^{2} \mathrm{Gr}_{-1}^{W} \mathfrak{u}_{g, n}^{\text {geom }} \rightarrow \mathrm{Gr}_{-2}^{W} \mathfrak{u}_{g, n}^{\text {geom }} .
$$

We do this by describing its "matrix entries". To this end, write

$$
\operatorname{Gr}_{-1}^{W} \mathfrak{u}_{g, n}^{\mathrm{geom}}=\Lambda_{n}^{3} H=\Lambda_{0}^{3} H \oplus H_{1} \oplus \cdots \oplus H_{n},
$$

where the $j$ th copy $H_{j}$ of $H$ corresponds to the $j$ th point. Write

$$
\mathrm{Gr}_{-2}^{W} \mathfrak{u}_{g, n}^{\text {geom }}=V_{\boxplus} \oplus \bigoplus_{i<j} \mathbb{Q}_{\ell}(1)_{i j} \oplus \bigoplus_{j=1}^{n} \Lambda_{0}^{2} H_{j} .
$$

This isomorphism is chosen so that the bracket

$$
\Lambda^{2} H_{j} \hookrightarrow \mathrm{Gr}_{-2}^{W} \mathfrak{u}_{g, n}^{\text {geom }} \stackrel{\text { proj }}{\rightarrow} \Lambda_{0}^{2} H_{j}
$$

is the quotient mapping and so that

$$
H \otimes H \cong H_{i} \otimes H_{j} \hookrightarrow \mathrm{Gr}_{-2}^{W} \mathfrak{u}_{g, n}^{\text {geom }} \stackrel{\text { proj }}{\rightarrow} \mathbb{Q}_{\ell}(1)_{i j}
$$

is the polarization $\theta$. That this is possible follows from [15, §13]. Let

$$
p_{j}: \mathrm{Gr}_{-2}^{W} \mathfrak{u}_{g, n}^{\text {geom }} \rightarrow \Lambda_{0}^{2} H, q_{i j}: \mathrm{Gr}_{-2}^{W} \mathfrak{u}_{g, n}^{\text {geom }} \rightarrow \mathbb{Q}_{\ell}(1), p_{\boxplus}: \mathrm{Gr}_{-2}^{W} \mathfrak{u}_{g, n}^{\text {geom }} \rightarrow V_{\boxplus}
$$

be the corresponding projections.

Note that

$$
\Lambda^{2} \operatorname{Gr}_{-1}^{W} \mathfrak{u}_{g, n}^{\text {geom }} \cong \Lambda^{2} \Lambda_{0}^{3} H \oplus \bigoplus_{j=1}^{n}\left(H_{j} \otimes \Lambda_{0}^{3} H\right) \oplus \bigoplus_{j=1}^{n} \Lambda^{2} H_{j} \oplus \bigoplus_{i<j} H_{i} \otimes H_{j} .
$$

Choose $\operatorname{GSp}(H)$-equivariant projections

$$
\begin{gathered}
\text { c }: \Lambda^{2} \Lambda_{0}^{3} H \rightarrow \Lambda_{0}^{2} H, \mathbf{d}: H \otimes \Lambda_{0}^{3} H \rightarrow \Lambda_{0}^{2} H, \\
\mathbf{e}: \Lambda^{2} H \rightarrow \Lambda_{0}^{2} H, \psi: \Lambda^{2} \Lambda_{0}^{3} H \rightarrow \mathbb{Q}_{\ell}(1) .
\end{gathered}
$$


Here, and in the following definition, $g \geq 3$ except in the definition of $\mathbf{c}$ where $g \geq 4$. Proposition 9.5 implies that each is unique up to a scalar multiple. Denote the $\operatorname{GSp}(H)$-invariant projections

$$
\begin{aligned}
& \Lambda^{2} \mathrm{Gr}_{-1}^{W} \mathfrak{u}_{g, n}^{\text {geom }} \rightarrow \Lambda^{2} \Lambda_{0}^{3} H \stackrel{\mathbf{c}}{\rightarrow} \Lambda_{0}^{2} H \quad g \geq 4 \\
& \Lambda^{2} \mathrm{Gr}_{-1}^{W} \mathfrak{u}_{g, n}^{\text {geom }} \rightarrow H_{j} \otimes \Lambda_{0}^{3} H \stackrel{\text { d }}{\rightarrow} \Lambda_{0}^{2} H \\
& \Lambda^{2} \mathrm{Gr}_{-1}^{W} \mathfrak{u}_{g, n}^{\text {geom }} \rightarrow \Lambda^{2} H_{j} \stackrel{\text { e }}{\rightarrow} \Lambda_{0}^{2} H \\
& \Lambda^{2} \mathrm{Gr}_{-1}^{W} \mathfrak{u}_{g, n}^{\text {geom }} \rightarrow H_{i} \otimes H_{j} \stackrel{\text { e }}{\rightarrow} \Lambda_{0}^{2} H \\
& \Lambda^{2} \mathrm{Gr}_{-1}^{W} \mathfrak{u}_{g, n}^{\text {geom }} \rightarrow \Lambda^{2} H_{i} \stackrel{\theta}{\rightarrow} \mathbb{Q}_{\ell}(1) \\
& \Lambda^{2} \mathrm{Gr}_{-1}^{W} \mathfrak{u}_{g, n}^{\text {geom }} \rightarrow H_{i} \otimes H_{j} \stackrel{\theta}{\rightarrow} \mathbb{Q}_{\ell}(1) \\
& \Lambda^{2} \mathrm{Gr}_{-1}^{W} \mathfrak{u}_{g, n}^{\text {geom }} \rightarrow \Lambda^{2} \Lambda_{0}^{3} H \stackrel{\psi}{\rightarrow} \mathbb{Q}_{\ell}(1)
\end{aligned}
$$

by $\mathbf{c}, \mathbf{d}_{j}, \mathbf{e}_{j}, \mathbf{e}_{i j}, \theta_{i}, \theta_{i j}$ and $\psi$, respectively. For convenience, we define $\mathbf{c}$ to be zero when $g=3$. Proposition 9.5 and (11) imply:

Proposition 9.12. If $g \geq 3$ and $n \geq 0$, then

$$
\operatorname{Hom}_{\mathrm{Sp}(H)}\left(\Lambda^{2} \operatorname{Gr}_{-1}^{W} \mathfrak{u}_{g, n}^{\text {geom }}, \Lambda_{0}^{2} H\right)
$$

has basis

$$
\begin{array}{rr}
\left\{\mathbf{d}_{1}, \ldots, \mathbf{d}_{n}, \mathbf{e}_{1}, \ldots, \mathbf{e}_{n}\right\} \cup\left\{\mathbf{e}_{i j}: 1 \leq i<j \leq n\right\} & \text { for } g=3, \\
\left\{\mathbf{c}, \mathbf{d}_{1}, \ldots, \mathbf{d}_{n}, \mathbf{e}_{1}, \ldots, \mathbf{e}_{n}\right\} \cup\left\{\mathbf{e}_{i j}: 1 \leq i<j \leq n\right\} & \text { for } g \geq 4
\end{array}
$$

and

$$
\operatorname{Hom}_{\mathrm{Sp}(H)}\left(\Lambda^{2} \mathrm{Gr}_{-1}^{W} \mathfrak{u}_{g, n}^{\text {geom }}, \mathbb{Q}_{\ell}\right)
$$

has basis

$$
\left\{\psi, \theta_{i j}: 1 \leq i<j \leq n\right\} \cup\left\{\theta_{1}, \ldots, \theta_{n}\right\}
$$

for all $g \geq 3$.

The bracket of $\mathrm{Gr}_{\bullet}^{W} \mathfrak{u}_{g, n}^{\text {geom }}$ is determined by:

Proposition 9.13. If $g \geq 3$, then, after rescaling $\psi$, $\mathbf{c}$ and $\mathbf{d}$ by non-zero constants if necessary 13

$$
\begin{aligned}
& p_{j} \circ \text { bracket }=\mathbf{d}_{j}+\mathbf{e}_{j} \quad \text { for } g=3, \\
& p_{j} \circ \text { bracket }=\mathbf{c}+\mathbf{d}_{j}+\mathbf{e}_{j} \quad \text { for } g \geq 4, \\
& q_{i j} \circ \text { bracket }=\psi+\theta_{i j} \quad \text { for } g \geq 3 .
\end{aligned}
$$

In addition, $p_{\boxplus} \circ$ bracket is non-zero and vanishes on each $H_{j} \otimes \Lambda_{0}^{3} H$ and each $H_{i} \otimes H_{j}$, where $i \leq j$.

The bracket of $\mathrm{Gr}_{\bullet}^{W} \hat{\mathfrak{u}}_{g, n}^{\text {geom }}$ is obtained by ignoring the $\mathbb{Q}_{\ell}(1)$ factors.

\footnotetext{
${ }^{13}$ Explicit formulas for these projections can be deduced from the formulas in [15, §11], although we shall not need them.
} 
Proof of Theorem 9.11 and Proposition 9.13. Lemma 8.7. Theorem 9.7 and Proposition 8.6 imply that

$$
\mathrm{Gr}_{-1}^{W} \mathfrak{u}_{g, n}^{\text {geom }} \cong \mathrm{Gr}_{-1}^{W} \hat{\mathfrak{u}}_{g, n}^{\text {geom }} \cong \Lambda_{n}^{3} H
$$

and that

$$
\mathrm{Gr}_{-2}^{W} \hat{\mathfrak{u}}_{g, n}^{\text {geom }} \cong V_{\boxplus} \oplus\left(\Lambda_{0}^{2} H\right)^{n} \text { and } \operatorname{Gr}_{-2}^{W} \mathfrak{u}_{g, n}^{\text {geom }} \cong V_{\boxplus} \oplus\left(\Lambda_{0}^{2} H\right)^{n} \oplus \mathbb{Q}_{\ell}(1)^{\left(\begin{array}{l}
n \\
2
\end{array}\right) .}
$$

The bracket of $\mathrm{Gr}_{\bullet}^{W} \hat{\mathfrak{u}}_{g, n}^{\text {geom }} / W_{-3}$ is computed using the homomorphism

$$
\mathrm{Gr}_{\bullet}^{W} \hat{\mathfrak{u}}_{g, n}^{\mathrm{geom}} \rightarrow\left(\mathrm{Gr}_{\bullet}^{W} \hat{\mathfrak{u}}_{g, 1}^{\text {geom }}\right)^{n}
$$

induced by the inclusion $\mathcal{C}_{g / k}^{n} \rightarrow\left(\mathcal{M}_{g, 1 / k}\right)^{n}$, which is injective. The computation of the bracket in this case follows directly from Proposition 9.9. The surjectivity of $\mathfrak{u}_{g, n}^{\text {geom }} \rightarrow \mathfrak{u}_{g, n}^{\text {geom }}$ implies that, to compute the bracket in $\mathrm{Gr}_{\bullet}^{W} \mathfrak{u}_{g, n}^{\text {geom }} / W_{-3}$, we need only compute the $\mathbb{Q}_{\ell}(1)$ component. This is determined by the bracket in $\operatorname{Gr}_{\bullet}^{W} \mathfrak{p}_{g, n}$, which is computed in [15, §12].

\section{The Lie Algebra $\mathfrak{d}_{g, n}$}

In this section we associate a graded, 2-step nilpotent Lie algebra $\mathfrak{d}\left(\mathfrak{u}_{T}\right)$ to the base $T$ of a family of smooth projective curves with Zariski dense monodromy representation $\pi_{1}\left(T, t_{o}\right) \rightarrow \operatorname{GSp}\left(H_{\mathbb{Q}_{\ell}}\right)$. This Lie algebra is a useful tool for studying the existence of rational points. When applied to $\mathcal{M}_{g, n / k}$ or $\mathcal{C}_{g / k}^{n}$, it gives the Lie algebra $\mathfrak{d}_{g, n}$. The main result of this section is that, when $g \geq 4$, sections of the natural projection $\mathfrak{d}_{g, n+1} \rightarrow \mathfrak{d}_{g, n}$ correspond exactly to the $n$ tautological sections of the universal curve over $\mathcal{M}_{g, n / k}$.

Unless mentioned to the contrary, all Lie algebras in this section will be over the coefficient field $F$, which has characteristic zero. The most common choices in this work are $F=\mathbb{Q}, \mathbb{Q}_{\ell}, \mathbb{C}$.

10.1. The functor $\mathfrak{d}$ and the Lie algebra $\mathfrak{d}_{g, n}$. Suppose that $G$ is an extension of $\operatorname{GSp}(H)$ by a prounipotent group that is negatively weighted with respect to the standard cocharacter $\omega$, such as $\mathcal{G}_{g, n}$.

For a Lie algebra $\mathfrak{u}$ in the category of $G$-modules, define $\mathfrak{d}(\mathfrak{u})$ to be the Lie algebra

$$
\mathfrak{d}(\mathfrak{u})=\left(\operatorname{Gr}_{\bullet}^{W}\left(W_{-1} \mathfrak{u} / W_{-3}\right)\right) /\left(\Lambda_{0}^{2} H\right)^{\perp},
$$

where $\left(\Lambda_{0}^{2} H\right)^{\perp}$ denotes the $\operatorname{GSp}(H)$-invariant complement in $\mathrm{Gr}_{-2}^{W} \mathfrak{u}$ of its $\Lambda_{0}^{2} H$ isotypical component. It is a two-step, graded, nilpotent Lie algebra in the category of $\operatorname{GSp}(H)$-modules whose $r$ th graded quotient is

$$
\mathfrak{d}(\mathfrak{u})_{r} \begin{cases}\operatorname{Gr}_{-1}^{W} \mathfrak{u} & r=-1, \\ \operatorname{Gr}_{-2}^{W} \mathfrak{u} /\left(\Lambda_{0}^{2} H\right)^{\perp} & r=-2, \\ 0 & r \neq-1,-2 .\end{cases}
$$

Definition 10.1. Suppose that $g \geq 3$ and $n \geq 0$. Define $\mathfrak{d}_{g, n}=\mathfrak{d}\left(\mathfrak{u}_{g, n}^{\text {geom }}\right)$. This is a Lie algebra in the category of $S_{n} \times \mathrm{GSp}(H)$-modules.

This satisfies

$$
\left(\mathfrak{d}_{g, n}\right)_{r}= \begin{cases}\Lambda_{n}^{3} H & r=-1 \\ \left(\Lambda_{0}^{2} H\right)^{n} & r=-2 .\end{cases}
$$

Note also that $\mathfrak{d}_{g, 0}$ is an abelian Lie algebra isomorphic to $\Lambda_{0}^{3} H$. 
Proposition 8.8 implies that for all $g \geq 3$ and $n \geq 0$, the natural map $\mathfrak{u}_{g, n}^{\text {geom }} \rightarrow$ $\mathfrak{u}_{g, n}$ induces an $S_{n} \times \operatorname{GSp}(H)$-equivariant Lie algebra isomorphism

$$
\mathfrak{d}_{g, n}=\mathfrak{d}\left(\mathfrak{u}_{g, n}^{\text {geom }}\right) \cong \mathfrak{d}\left(\mathfrak{u}_{g, n}\right) .
$$

Denote the universal complete curve over $\mathcal{M}_{g, n / k}$ by $\mathcal{C}_{g, n / k}$. This is a Zariski open subset of the $(n+1)$ st power $\mathcal{C}_{g / k}^{n+1}$ of the universal curve over $\mathcal{M}_{g / k}$. Index the copies of the universal curve by integers $j$ between 0 and $n$. Label the points so that the projection $\mathcal{C}_{g, n / k} \rightarrow \mathcal{M}_{g, n / k}$ takes $\left[C ; x_{0}, \ldots, x_{n}\right]$ to $\left[C ; x_{1}, \ldots, x_{n}\right]$. This projection is $S_{n}$-equivariant, where $S_{n}$ acts by permuting the points $\left\{x_{1}, \ldots, x_{n}\right\}$. Note that $\mathcal{M}_{g, n+1 / k}$ is a Zariski open subset of $\mathcal{C}_{g, n / k}$. A useful property of the functor $\mathfrak{d}$, which is proved below, is that, when $g \geq 3$, the inclusion $\mathcal{M}_{g, n+1} \hookrightarrow \mathcal{C}_{g, n}$ induces an isomorphism on $\mathfrak{d}(\mathfrak{u})$.

Choose a geometric point $\bar{\eta}$ of $\mathcal{M}_{g, n+1 / k}$. Assume that $\chi_{\ell}: G_{k} \rightarrow \mathbb{Z}_{\ell}^{\times}$has infinite image. Denote the Lie algebra of the weighted completion of $\pi_{1}\left(\mathcal{C}_{g, n / k}, \bar{\eta}\right)$ with respect to the monodromy representation to $\operatorname{GSp}(H)$ by $\mathfrak{g}_{\mathcal{C}_{g, n}}$ and the Lie algebra of its prounipotent radical by $\mathfrak{u}_{\mathcal{C}_{g, n}}$. Denote the Lie algebra of the relative completion of $\pi_{1}\left(\mathcal{C}_{g, n / \bar{k}}, \bar{\eta}\right)$ by $\mathfrak{g}_{\mathcal{C}_{g, n}}^{\text {geom }}$ and the Lie algebra of its prounipotent radical by $\mathfrak{u}_{\mathcal{C}_{g, n}}^{\text {geom }}$.

Proposition 10.2. If $g \geq 3$ and $n \geq 0$, then the inclusion $\mathcal{M}_{g, n+1} \hookrightarrow \mathcal{C}_{g, n / k}$ induces $\operatorname{GSp}(H)$-equivariant Lie algebra isomorphisms

$$
\mathfrak{d}_{g, n+1}:=\mathfrak{d}\left(\mathfrak{u}_{g, n+1}^{\text {geom }}\right) \cong \mathfrak{d}\left(\mathfrak{u}_{g, n+1}\right) \cong \mathfrak{d}\left(\mathfrak{u}_{\mathcal{C}_{g, n}}^{\text {geom }}\right) \cong \mathfrak{d}\left(\mathfrak{u}_{\mathcal{C}_{g, n}}\right)
$$

The first isomorphism is $S_{n+1}$-equivariant; the remaining two isomorphisms are $S_{n}$-equivariant.

Proof. We first prove the arithmetic case. Consider the diagram

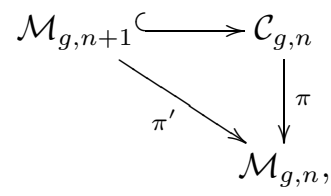

where $\pi$ and $\pi^{\prime}$ both take $\left(x_{0}, \ldots, x_{n}\right)$ to $\left(x_{1}, \ldots, x_{n}\right)$. The fiber of $\pi^{\prime}$ is an $n$ punctured curve. Choose compatible basepoints for all three spaces. Denote the unipotent completion of the fiber of $\pi^{\prime}$ over the basepoint of $\mathcal{M}_{g, n}$ by $\mathfrak{p}^{\prime}$. Then one has the commutative diagram

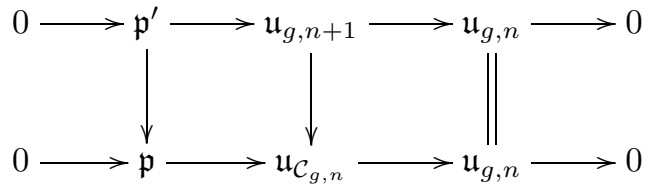

whose vertical maps are surjective. Exactness of the second row follows from Proposition 7.1, exactness of the first row follows from Proposition 6.11 because $\mathfrak{p}^{\prime}$ is free and therefore has trivial center, and because $H_{1}(\mathfrak{p})$ has weights -1 and -2 . The diagram implies that

$$
\mathrm{Gr}_{-2}^{W} \operatorname{ker}\left\{\mathfrak{u}_{g, n+1} \rightarrow \mathfrak{u}_{\mathcal{C}_{g, n}}\right\}=\mathrm{Gr}_{-2}^{W} \operatorname{ker}\left\{\mathfrak{p}^{\prime} \rightarrow \mathfrak{p}\right\} \cong \mathbb{Q}_{\ell}(1)^{n-1}
$$

from which it follows that $\mathfrak{d}\left(\mathfrak{u}_{\mathcal{C}_{g, n}}\right) \cong \mathfrak{d}\left(\mathfrak{u}_{g, n+1}\right) \cong \mathfrak{d}_{g, n+1}$. The proof that $\mathfrak{d}\left(\mathfrak{u}_{\mathcal{C}_{g, n}}^{\text {geom }}\right) \cong$ $\mathfrak{d}\left(\mathfrak{u}_{g, n+1}^{\text {geom }}\right)$ is similar and is left to the reader. The result now follows from (13). 
Corollary 10.3. Each section $x$ of the universal curve $\pi: \mathcal{C}_{g, n / k} \rightarrow \mathcal{M}_{g, n / k}$ induces a well-defined section of $\mathfrak{d}_{g, n+1} \rightarrow \mathfrak{d}_{g, n}$.

Proof. Proposition 7.1 implies that each section $x$ of the universal curve induces a section $\sigma_{x}$ of $\pi_{*}: \mathcal{G}_{\mathcal{C}_{g, n}} \rightarrow \mathcal{G}_{g, n}$ that is well defined up to conjugation by an element of $\mathcal{P}:=\operatorname{ker} \pi_{*}$. The section $x$ therefore induces a section $d \sigma_{x}$ of $d \pi_{*}: \mathfrak{g}_{\mathcal{C}_{g, n}} \rightarrow \mathfrak{g}_{g, n}$ which is well defined up to the addition of a section of the form $\operatorname{ad}_{u} \circ \sigma_{x}$, where $u \in \mathfrak{p}:=\operatorname{ker} d \pi_{*}$. Since $\mathfrak{p}=W_{-1} \mathfrak{p}$, the sections $d \sigma_{x}$ and $d \sigma+\operatorname{ad}_{u} \circ \sigma_{x}$ induce the same section of $\mathrm{Gr}_{\bullet}^{W} \mathfrak{g}_{\mathcal{C}_{g, n}} \rightarrow \mathrm{Gr}_{\bullet}^{W} \mathfrak{g}_{g, n}$, and therefore of $\mathrm{Gr}_{\bullet}^{W} \mathfrak{u}_{\mathcal{C}_{g, n}} / W_{-3} \rightarrow$ $\mathrm{Gr}_{\bullet}^{W} \mathfrak{u}_{g, n} / W_{-3}$. The result now follows from Proposition 10.2.

Equality with the geometric case follows from equation (13) for $\mathcal{M}_{g, n+1}$.

10.2. Splittings. Elements of $\Lambda_{n}^{3} H$ will be denoted by $\left(v ; u_{1}, \ldots, u_{n}\right)$, where $v \in$ $\Lambda_{0}^{3} H$ and $u_{j} \in H$. When the role of $v$ is clear, it will be omitted. For all $n \geq 0$, the linear mapping

$$
\mathrm{Gr}_{-1}^{W} \mathfrak{d}_{g, n+1} \rightarrow \mathrm{Gr}_{-1}^{W} \mathfrak{d}_{g, n}
$$

defined by $\left(u_{0}, \ldots, u_{n}\right) \mapsto\left(u_{1}, \ldots, u_{n}\right)$ induces a $\operatorname{GSp}(H)$-equivariant homomorphism $\epsilon_{n}: \mathfrak{d}_{g, n+1} \rightarrow \mathfrak{d}_{g, n}$.

Proposition 10.4. If $g \geq 4$, then there are exactly $n \mathrm{GSp}(H)$-invariant sections of $\epsilon_{n}$. When $n \geq 1$, these are defined by

$$
s_{j}:\left(u_{1}, \ldots, u_{n}\right) \mapsto\left(u_{j}, u_{1}, \ldots, u_{n}\right), \quad j=1, \ldots, n .
$$

When $g=3$, the sections of $\epsilon_{n}$ are $s_{1}, \ldots, s_{n}$ and the section

$$
\zeta_{n}:\left(u_{1}, \ldots, u_{n}\right) \mapsto\left(0, u_{1}, \ldots, u_{n}\right) .
$$

Proof. Suppose that $n \geq 1$ and that $g \geq 3$. Suppose that $s: \mathfrak{d}_{g, n} \rightarrow \mathfrak{d}_{g, n+1}$ is a $\operatorname{GSp}(H)$-invariant section of $\epsilon_{n}: \mathfrak{d}_{g, n+1} \rightarrow \mathfrak{d}_{g, n}$. Since $H$ is an irreducible $\operatorname{GSp}(H)$ module, Schur's Lemma implies that the restriction of $s$ to the subgroup $H^{n}$ of $\mathfrak{d}_{g, n}$ is of the form

$$
s\left(u_{1}, \ldots, u_{n}\right)=\left(\sum_{j=1}^{n} a_{j} u_{j}, u_{1}, \ldots, u_{n}\right)
$$

for some $a_{1}, \ldots, a_{n} \in F$. Since $\epsilon_{n}$ is the identity on $\Lambda_{0}^{3} H, s$ is as well. So, since the bracket

$$
H_{j} \otimes \Lambda_{0}^{3} H \rightarrow \Lambda_{0}^{2} H_{j}, \quad 1 \leq j \leq n
$$

is surjective, the map

$$
\Lambda_{0}^{2} H_{j} \rightarrow \Lambda_{0}^{2} H_{0}, \quad 1 \leq j \leq n
$$

induced by $s$ must also be multiplication by $a_{j}$.

Lemma 10.5. If $g \geq 3$ and $v^{\prime}, v^{\prime \prime} \in \Lambda_{0}^{3} H$ and $u_{1}^{\prime}, u_{1}^{\prime \prime}, \ldots, u_{n}^{\prime}, u_{n}^{\prime \prime} \in H$, then the component of

$$
\left[s\left(v^{\prime} ; u_{1}^{\prime}, \ldots, u_{n}^{\prime}\right), s\left(v^{\prime \prime} ; u_{1}^{\prime \prime}, \ldots, u_{n}^{\prime \prime}\right)\right]-s\left(\left[\left(v^{\prime} ; u_{1}^{\prime}, \ldots, u_{n}^{\prime}\right),\left(v^{\prime \prime} ; u_{1}^{\prime \prime}, \ldots, u_{n}^{\prime \prime}\right)\right]\right)
$$

in $\Lambda_{0}^{2} H_{0}$ is

$$
\left(1-\sum_{j} a_{i}\right) \mathbf{c}\left(v^{\prime} \wedge v^{\prime \prime}\right)+\sum_{j}\left(a_{j}^{2}-a_{j}\right) \mathbf{e}_{j}\left(u_{j}^{\prime} \wedge u_{j}^{\prime \prime}\right)+\sum_{i \neq j} a_{i} a_{j} \mathbf{e}_{i j}\left(u_{i}^{\prime} \wedge u_{j}^{\prime \prime}\right) .
$$

Remark 10.6. This result is reinterpreted in terms of non-abelian cohomology in Lemma 18.2 
Since $s$ is a section, the expression (14) must vanish. This implies that $a_{i}^{2}=a_{i}$ for all $i$ and that $a_{i} a_{j}=0$ when $i \neq j$. That is, each $a_{i} \in\{0,1\}$ and at most one $a_{i}$ is non-zero. When $g \geq 4, \mathbf{c} \neq 0$. Consequently, we have the additional equation

$$
\sum_{j=1}^{n} a_{j}=1,
$$

which implies that exactly one of the $a_{j}$ is non-zero. This proves the result when $g \geq 4$. When $g=3$, $\mathbf{c}$ is zero and we also have the section $\zeta_{n}$, where all $a_{j}$ are zero.

Proof of Lemma 10.5. Proposition 9.13 implies that

$$
\begin{aligned}
& p_{0}\left(\left[s\left(v^{\prime} ; u_{1}^{\prime}, \ldots, u_{n}^{\prime}\right), s\left(v^{\prime \prime} ; u_{1}^{\prime \prime}, \ldots, u_{n}^{\prime \prime}\right)\right]\right)=\mathbf{c}\left(v^{\prime} \wedge v^{\prime \prime}\right) \\
& +\sum_{j} a_{j}\left(\mathbf{d}_{j}\left(v^{\prime} \otimes u_{j}^{\prime \prime}\right)-\mathbf{d}_{j}\left(v^{\prime \prime} \otimes u_{j}^{\prime}\right)\right)+\sum_{j} a_{j}^{2} \mathbf{e}_{j}\left(u_{j}^{\prime} \wedge u_{j}^{\prime \prime}\right)+\sum_{i \neq j} a_{i} a_{j} \mathbf{e}_{i j}\left(u_{i}^{\prime} \wedge u_{j}^{\prime \prime}\right)
\end{aligned}
$$

and that

$$
\begin{aligned}
p_{0} \circ s\left(\left[\left(v^{\prime} ; u_{1}^{\prime}, \ldots, u_{n}^{\prime}\right),\left(v^{\prime \prime} ; u_{1}^{\prime \prime}, \ldots, u_{n}^{\prime \prime}\right)\right]\right) \\
\quad=\sum_{j} a_{j}\left(\mathbf{e}\left(u_{j}^{\prime} \wedge u_{j}^{\prime \prime}\right)+\mathbf{d}\left(v^{\prime} \otimes u_{j}^{\prime \prime}\right)-\mathbf{d}\left(v^{\prime \prime} \otimes u_{j}^{\prime}\right)+\mathbf{c}\left(v^{\prime} \wedge v^{\prime \prime}\right)\right) .
\end{aligned}
$$

The expression (14) is their difference.

10.3. Genus 3. As we have seen, the section $\zeta_{n}$ of $\mathfrak{d}_{g, n+1} \rightarrow \mathfrak{d}_{g, n}$ exists only when $g=3$. This exceptional section occurs because $\Lambda^{2} \Lambda_{0}^{3} H$ does not contain $\Lambda_{0}^{2} H$ when $g=3$. To circumvent this problem, we consider a natural integral structure on $\mathrm{Gr}_{-1}^{W} \mathfrak{u}_{g, n}$ that comes from the image of the Torelli group in $\mathcal{U}_{g, n}$. This will allow us to prove that $\zeta_{n}$ cannot be induced by a rational point.

Suppose that $A$ is an integral domain with fraction field $F$. Recall that

$$
\Lambda_{0}^{3} H_{A}:=\left(\Lambda^{3} H_{A}\right)(-1) / \check{\theta} \wedge H_{A} \cong \operatorname{im}\left\{\Lambda^{3} H_{A} \rightarrow \Lambda_{0}^{3} H_{F}\right\} .
$$

For a positive integer $n$, define a lattice in $\Lambda_{n}^{3} H_{F}$ by

$$
\Lambda_{n}^{3} H_{A}=\left\{\left(u_{1}, \ldots, u_{n}\right) \in\left(\Lambda^{3} H_{A}\right)^{n}: \bar{u}_{1}=\cdots=\bar{u}_{n}\right\}(-1) .
$$

Define the projection $r: \Lambda_{n+1}^{3} H_{A} \rightarrow \Lambda_{n}^{3} H_{A}$ by $r:\left(u_{0}, u_{1}, \ldots, u_{n}\right) \mapsto\left(u_{1}, \ldots, u_{n}\right)$.

The following is a straightforward generalization of Lemma 9.4 .

Lemma 10.7. Suppose that $g \geq 3$ and $n \geq 0$. If $A$ is an integral domain and $g-1 \notin A^{\times}$, then $\zeta_{n}$ does not restrict to a section of the projection $r: \Lambda_{n+1}^{3} H_{A} \rightarrow$ $\Lambda_{n}^{3} H_{A}$.

Now take the coefficient field $F$ to be $\mathbb{Q}_{\ell}$. The homomorphism (7) induces natural isomorphisms

$$
\mathrm{Gr}_{-1}^{W} \mathfrak{u}_{g, 1} \cong \Lambda_{1}^{3} H_{\mathbb{Q}_{\ell}} \cong\left(\Lambda_{1}^{3} H_{\mathbb{Z}_{\ell}}\right) \otimes \mathbb{Q}_{\ell} .
$$

These induce a natural isomorphism

$$
\mathrm{Gr}_{-1}^{W} \mathfrak{u}_{g, n} \cong\left(\Lambda_{n}^{3} H_{\mathbb{Z}_{\ell}}\right) \otimes \mathbb{Q}_{\ell}
$$

for all $n \geq 0$ such that the $j$ th projection $r: \Lambda_{n+1}^{3} H_{\mathbb{Z}_{\ell}} \rightarrow \Lambda_{n}^{3} H_{\mathbb{Z}_{\ell}}$ corresponds to the $j$ th projection $\mathrm{Gr}_{-1}^{W} \mathfrak{u}_{g, n+1} \rightarrow \mathrm{Gr}_{-1}^{W} \mathfrak{u}_{g, n}$. 
10.4. Summary. The following result summarizes the results of Section 10.

Proposition 10.8. Suppose that $n \geq 1$. If $g \geq 4$ and $\ell$ is arbitrary, or if $g=3$ and $\ell=2$, then the only $\operatorname{GSp}\left(H_{\mathbb{Q}_{\ell}}\right)$-invariant sections of $\epsilon_{n}: \mathfrak{d}_{g, n+1} \rightarrow \mathfrak{d}_{g, n}$ that respect the integral structure on $\mathrm{Gr}_{-1}^{W}$ are the sections $s_{1}, \ldots, s_{n}$ induced by the tautological points. In particular, there are no $\operatorname{GSp}(H)$-invariant sections of $\mathfrak{d}_{g, 1} \rightarrow \mathfrak{d}_{g, 0}$.

Proof. Corollary 10.3 implies that each tautological section induces a $\operatorname{GSp}(H)$ invariant section of $e_{n}$. When $n=1$, the result follows from Lemma 10.7 when $g=3$ and from Proposition 10.4 when $g \geq 4$. The case $n>1$ follows from the case $n=1$ and naturality.

\section{TOPOLOGICALly AMPLE FAMILIES OF CURVES}

Roughly speaking, a family $C \rightarrow T$ of smooth projective curves, where $T$ is a smooth, geometrically connected $k$-variety, is topologically ample of type $(g, n)$ if $C$ is the pullback of the universal curve along a map $T \rightarrow \mathcal{M}_{g, n / k}$ that induces an isomorphism on fundamental groups. The actual condition is weaker.

Definition 11.1. Suppose that $k$ is a field of characteristic zero and that $T$ is a smooth, geometrically connected $k$-variety. A family $C \rightarrow T$ of smooth projective curves of genus $g \geq 3$ is topologically ample over $k$ of type $(g, n)$ if there is a $k$ morphism $\phi: T \rightarrow \mathcal{M}_{g, n / k}$, called a marking, and a prime number $\ell$ satisfying:

(i) $C$ is isomorphic to the pullback of the universal curve $\mathcal{C} \rightarrow \mathcal{M}_{g, n / k}$; equivalently, there is a morphism $\hat{\phi}: C \rightarrow \mathcal{C}_{g, n / k}$ such that

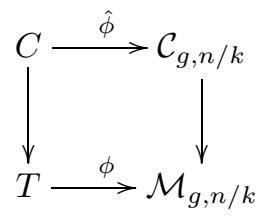

commutes;

(ii) the monodromy representation $\rho: \pi_{1}(T, \bar{\eta}) \rightarrow \operatorname{GSp}\left(H_{\mathbb{Q}_{\ell}}\right)$ is Zariski dense, where $\bar{\eta}$ is a geometric point of $T$ and $H=H_{\text {ét }}^{1}\left(C_{\bar{\eta}}, \mathbb{Q}_{\ell}(1)\right)$;

(iii) the Lie algebra homomorphism $\phi_{*}: \mathfrak{u}_{T} \rightarrow \mathfrak{u}_{g, n}$ induced by $\phi$ induces an isomorphism $\mathfrak{u}_{T} / W_{-3} \rightarrow \mathfrak{u}_{g, n} / W_{-3}$, where $\mathfrak{u}_{T}$ and $\mathfrak{u}_{g, n}$ denote the Lie algebras of the prounipotent radicals of the weighted completions of $\pi_{1}(T, \bar{x})$ and $\pi_{1}\left(\mathcal{M}_{g, n / k}, \phi(\bar{x})\right)$ with respect to their homomorphisms to $\operatorname{GSp}\left(H_{\mathbb{Q}_{\ell}}\right)$

(iv) the group of sections $\operatorname{Jac}_{C / T}(T)$ of the relative Jacobian $\operatorname{Jac}_{C / T} \rightarrow T$ is finitely generated.

When $g=3$, we also require that $\ell=2$ and that the image of the natural homomorphism

$$
\operatorname{ker}\left\{\pi_{1}(T, \bar{\eta}) \rightarrow \operatorname{GSp}\left(H_{\mathbb{Q}_{2}}\right)\right\} \rightarrow \operatorname{Gr}_{-1}^{W} \mathfrak{u}_{g, n}
$$

equals the copy of $\Lambda_{n}^{3} H_{\mathbb{Z}_{2}}$ defined in Paragraph 10.3. A marked topologically ample curve of type $(g, n)$ is a topologically ample curve $C \rightarrow T$ of type $(g, n)$ together with a choice of marking $\phi: T \rightarrow \mathcal{M}_{g, n / k}$.

Every marked topologically ample family of curves $C \rightarrow T$ of type $(g, n)$ has $n$ tautological points $x_{1}, \ldots, x_{n}$. These are the pullbacks of the $n$ tautological sections of the universal curve over $\mathcal{M}_{g, n / k}$ along the marking $\phi$. 
Finite generation of $\operatorname{Jac}_{C / T}(T)$ occurs when $k$ is small and also when the image of $\pi_{1}\left(T \otimes_{k} \bar{k}, \bar{\eta}\right) \rightarrow \operatorname{GSp}\left(H_{\mathbb{Q}_{\ell}}\right)$ is large enough. It fails, for example, when $k$ is algebraically closed and $T=\operatorname{Spec} k$.

Proposition 11.2. Suppose that $T$ is a geometrically connected, smooth k-variety. If either $k$ is finitely generated over $\mathbb{Q}$ or if $H^{0}\left(T \otimes_{k} \bar{k}, \mathbb{H}_{\mathbb{Q}_{\ell}}\right)=0$, then $\operatorname{Jac}_{C / T}(T)$ is finitely generated.

Proof. The case when $k$ is finitely generated over $\mathbb{Q}$ follows from the Mordell-Weil Theorem [44. To prove the second assertion, it suffices, by standard arguments, to prove it in the case $k=\mathbb{C}$. Denote the category of mixed Hodge structures (MHS) by $\mathcal{H}$ and the category of admissible variations of MHS over $T$ by $\mathcal{H}(T)$. In this case, the Abel-Jacobi mapping

$$
\operatorname{Jac}_{C / T}(T) \rightarrow \operatorname{Ext}_{\mathcal{H}(T)}^{1}\left(\mathbb{Z}(0)_{T}, \mathbb{H}_{\mathbb{Z}}\right)
$$

is injective. It is proved in the Appendix that the sequence

$$
0 \rightarrow \operatorname{Ext}_{\mathcal{H}}^{1}\left(\mathbb{Z}(0), H^{0}\left(T, \mathbb{H}_{\mathbb{Z}}\right)\right) \rightarrow \operatorname{Ext}_{\mathcal{H}(T)}^{1}\left(\mathbb{Z}(0)_{T}, \mathbb{H}_{\mathbb{Z}}\right) \rightarrow H^{1}\left(T, \mathbb{H}_{\mathbb{Z}}\right)
$$

is exact. Since $\mathbb{H}_{\mathbb{Z}}$ is torsion free, $H^{0}\left(T, \mathbb{H}_{\mathbb{Z}}\right)$ is a subgroup of $H^{0}\left(T, \mathbb{H}_{\mathbb{Q}_{\ell}}\right)$, and therefore vanishes. Since $T$ is homotopy equivalent to a finite complex, $H^{1}\left(T, \mathbb{H}_{\mathbb{Z}}\right)$ is finitely generated. It follows that $\operatorname{Jac}_{C / T}(T)$ is finitely generated.

Proposition 8.2 implies that when $g \geq 4$, the universal curve over $\mathcal{M}_{g, n / k}[m]$ is topologically ample whenever there is a prime number $\ell$ such that $\chi_{\ell}: G_{k} \rightarrow \mathbb{Z}_{\ell}^{\times}$ has infinite image. When $g=3$, this implies that $\mathcal{M}_{3, n / k}[\mathrm{~m}]$ is topologically ample when the image of $\chi_{2}$ is infinite. This condition is satisfied, for example, when $k$ is a number field. Recall that $\mathcal{M}_{g, n}[m]$ denotes the open subset of $\mathcal{M}_{g, n}[m]$ of curves with trivial automorphism group. As indicated in Section [5.1.2, it is a smooth quasi-projective variety whenever $g \geq 3$.

Proposition 11.3. Suppose that $g \geq 3, n \geq 0, m \geq 1$ and that either $g+n \geq$ 4 or $m \geq 3$. If there exists a prime number $\ell$ such that the $\ell$-adic cyclotomic character $\chi_{\ell}: G_{k} \rightarrow \mathbb{Z}_{\ell}^{\times}$has infinite image and if $T$ is a generic ample subvariety of $\mathcal{M}_{g, n / k}[m]$ of dimension $\geq 2$, then the pullback of the universal curve over $\mathcal{M}_{g, n}$ to $T$ is topologically ample of type $(g, n)$.

Proof. If $m \geq 3$, then $\mathcal{M}_{g, n}[m]=\mathcal{M}_{g, n}[m]$. Proposition 5.1 implies that if $g+n \geq 4$, then $\mathcal{M}_{g, n / k}[m]$ is a smooth quasi-projective variety whose geometric fundamental group is isomorphic to the profinite completion of the mapping class group $\Gamma_{g, n}[m]$. Choose a projective imbedding $\mathcal{M}_{g, n / k}[m] \hookrightarrow \mathbb{P}_{k}^{N}$. Denote the Zariski closure of the image by $X$. Set

$$
r=N-(3 g-3+n)+d .
$$

When $d \geq 0$, there is a Zariski open subset $U_{d}$ of the Grassmannian $G_{r}\left(\mathbb{P}^{N}\right)$ consisting of the $r$ planes in $\mathbb{P}^{N}$ that intersect $X$ and all of the strata of $X-\mathcal{M}_{g, n}$ transversely. If $L \in U_{d}(k)$, then $L \cap X$ is a subvariety of $X$ of dimension $d$.

Observe that $U_{d}(k)$ is non-empty. If $d \geq 2$ and $L \in U_{d}(k)$, then, by [10, Thm. p. 150], $\mathcal{M}_{g, n} \cap L$ is a geometrically connected $k$-variety of dimension $d$ and the inclusion

$$
T:=\stackrel{\mathcal{M}}{g, n / k}_{k}[m] \cap L \hookrightarrow \mathcal{M}_{g, n / k}[m]
$$


induces an isomorphism on geometric fundamental groups. It thus induces an isomorphism on étale fundamental groups. Let $C \rightarrow T$ be the restriction of the universal curve over $\mathcal{M}_{g, n / k}$ to $T$. Since the $\ell$-adic cyclotomic character $\chi_{\ell}$ has infinite image, this implies that the representations $\pi_{1}(T) \rightarrow \operatorname{GSp}(H)$ and $\pi_{1}\left(C \otimes_{k}\right.$ $\bar{k}) \rightarrow \mathrm{Sp}(H)$ are Zariski dense. Proposition 11.2 implies that $\operatorname{Jac}_{C / T}(T)$ is finitely generated. It follows that $C / T$ is topologically ample of type $(g, n)$.

Remark 11.4. Suppose that $C / T$ is topologically ample of type $(g, n)$ with $g \geq 3$. Denote the lisse sheaf of $\mathbb{Q}_{\ell}$ vector spaces over $T$ that corresponds to the $\operatorname{GSp}(H)$ module $V$ by $\mathbb{V}$. Proposition 6.8 implies that if $V$ has negative weight, then one has isomorphisms

$$
\begin{aligned}
H_{\text {èt }}^{1}(T, \mathbb{V}) \cong \operatorname{Hom}_{\mathrm{GSp}(H)}\left(H_{1}\left(\mathfrak{u}_{T}\right), V\right) & \\
& \cong \operatorname{Hom}_{\mathrm{GSp}(H)}\left(H_{1}\left(\mathfrak{u}_{g, n}\right), V\right) \cong H_{\text {êt }}^{1}\left(\mathcal{M}_{g, n / k}, \mathbb{V}\right) .
\end{aligned}
$$

In particular, $H_{\text {ét }}^{1}\left(T, \mathbb{Q}_{\ell}(1)\right) \cong H_{\text {ét }}^{1}\left(\mathcal{M}_{g, n / k}, \mathbb{Q}_{\ell}(1)\right)=0$.

Recall that $\mathcal{C}_{g, n / k}$ denotes the universal complete curve over $\mathcal{M}_{g, n / k}$.

Proposition 11.5. If $C / T$ is topologically ample over $k$ of type $(g, n)$, then $\hat{\phi}$ induces an isomorphism $\hat{\phi}_{*}: \mathfrak{u}_{C} / W_{-3} \stackrel{\widetilde{\Xi}}{\rightarrow} \mathfrak{u}_{\mathcal{C}_{g, n / k}} / W_{-3}$ such that the diagram

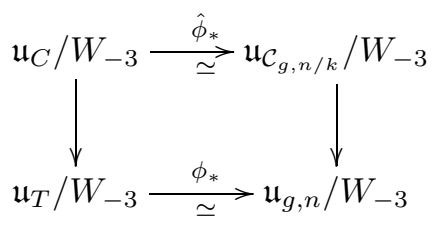

commutes.

Proof. This an immediate consequence of the commutativity of the diagram

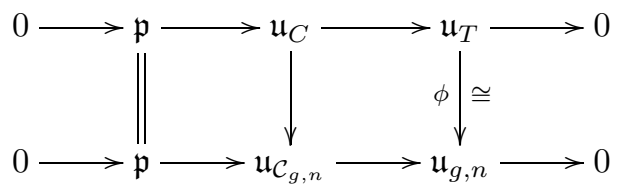

whose rows are exact by Proposition 7.1

The homomorphism $\phi_{*}: \mathfrak{u}_{T} \rightarrow \mathfrak{u}_{g, n}$ induces an isomorphism $\phi_{*}: \mathfrak{d}\left(\mathfrak{u}_{T}\right) \rightarrow \mathfrak{d}_{g, n}$. The next result is a direct consequence of Propositions 10.2 and 11.5. The proof of the last assertion is almost identical to that of Corollary 10.3 and is left to the reader.

Corollary 11.6. If $C / T$ is topologically ample over $k$ of type $(g, n)$, then there is $a \operatorname{GSp}(H)$-equivariant isomorphism $\mathfrak{d}\left(\mathfrak{u}_{C}\right) \cong \mathfrak{d}_{g, n+1}$ such that the diagram

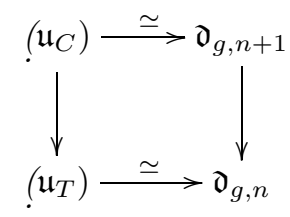

commutes. Each section $x \in C(T)$ induces a $\operatorname{GSp}(H)$-invariant section of $\mathfrak{d}\left(\mathfrak{u}_{C}\right) \rightarrow$ $\mathfrak{d}\left(\mathfrak{u}_{T}\right)$ which preserves the integral lattice on $\mathrm{Gr}_{-1}^{W}$. 


\section{The ClAss OF A RATIONAL POINT}

In this section we associate a cohomology class $\kappa_{x} \in H^{1}(T, \mathbb{H})$ to a section $x \in C(T)$ of a family of smooth projective curves $C \rightarrow T$. In the universal case, viz., the universal curve over $\mathcal{M}_{g, 1}$, this class is easily expressed in terms of the $\operatorname{GSp}(H)$-structure of $\mathrm{Gr}_{-1}^{W} \mathfrak{u}_{g, 1}$.

Suppose that $T$ is a smooth, geometrically connected $k$-scheme and that $f$ : $X \rightarrow T$ is a family of smooth projective varieties of relative dimension $d$ over $T$. The polarization gives an isomorphism $R^{1} f_{*} \mathbb{Q}_{\ell}(1) \cong R^{2 d-1} f_{*} \mathbb{Q}_{\ell}(d)$. By well-known arguments (cf. [22, $\S 4]$ ), each relative algebraic cycle $\xi$ in $X$ of dimension 0 over $T$ that is homologically trivial on each geometric fiber of $f$ determines a well-defined class

$$
c_{\xi} \in H_{\text {ét }}^{1}\left(T, R^{2 d-1} f_{*} \mathbb{Q}_{\ell}(d)\right) \cong H_{\text {ét }}^{1}\left(T, R^{1} f_{*} \mathbb{Q}_{\ell}(1)\right) .
$$

In particular, if $x_{1}, \ldots, x_{n}$ are sections of a family $C \rightarrow T$ of smooth projective curves of genus $g \geq 3$ and $d_{1}, \ldots, d_{n}$ are integers which sum to zero, then we have the class of the relative divisor $\xi=\sum d_{j} x_{j}$.

Denote the relative canonical divisor of $C / T$ by $\omega_{C / T}$. If $x \in C(T)$, then one has the algebraic 0 -cycle class $(2 g-2) x-\omega_{C / T}$, which is homologically trivial on each geometric fiber. Its class

$$
\kappa_{x} \in H_{\text {ét }}^{1}\left(T, \mathbb{H}_{\mathbb{Q}_{\ell}}\right)
$$

is well defined. In particular, one has the class

$$
\kappa \in H_{\text {ét }}^{1}\left(\mathcal{M}_{g, 1 / k}, \mathbb{H}_{\mathbb{Q}_{\ell}}\right)
$$

of the tautological section of the universal curve over $\mathcal{M}_{g, 1 / k}$. It is universal in the sense that each $x \in C(T)$ is classified by a $k$-morphism $\phi: T \rightarrow \mathcal{M}_{g, 1 / k}$ and $\kappa_{x}=\phi^{*} \kappa$. Denote the class of the $j$ th tautological point of the universal curve over $\mathcal{M}_{g, n / k}$ by $\kappa_{j}$.

Proposition 12.1. If $g \geq 3, n \geq 0$, and $m \geq 1$, then for all fields of $k$ characteristic zero

$$
H_{\text {ét }}^{1}\left(\mathcal{M}_{g, n / k}[m], \mathbb{H}_{\mathbb{Q}_{\ell}}\right)=\mathbb{Q}_{\ell} \kappa_{1} \oplus \mathbb{Q}_{\ell} \kappa_{2} \oplus \cdots \oplus \mathbb{Q}_{\ell} \kappa_{n} \text {. }
$$

Proof. When $n=1$, this follows directly form [22, Thm. 6.9]. The general case follows easily from this, naturality, and the computation [14, Prop. 5.2].

If $\pi: A \rightarrow T$ is a family of polarized abelian varieties over a smooth $k$-variety $T$, then the Abel-Jacobi mapping induces a homomorphism

$$
A(T) \rightarrow H_{\text {ét }}^{1}\left(T, R^{2 g-1} \pi_{*} \mathbb{Q}_{\ell}(g)\right) \cong H_{\text {ét }}^{1}\left(T, R^{1} \pi_{*} \mathbb{Q}_{\ell}(1)\right) .
$$

A direct proof of Corollary 12.3 below was communicated to me by Minhyong Kim; the current formulation, using the lemma, was contributed by the referee.

Lemma 12.2. If $\pi: A \rightarrow T$ is a family of polarized abelian varieties over a Noetherian scheme $T$, then the kernel of the $\ell$-adic Abel-Jacobi map

$$
A(T) \rightarrow H_{\text {ét }}^{1}\left(T, R^{1} \pi_{*} \mathbb{Z}_{\ell}(1)\right)
$$

is the subgroup $\bigcap_{n} \ell^{n} A(T)$ of $\ell^{\infty}$-divisible points.

Proof. The connecting homomorphism of the long exact cohomology sequence associated to the exact sequence of étale sheaves

$$
0 \rightarrow A\left[\ell^{n}\right] \rightarrow A \stackrel{\ell^{n}}{\rightarrow} A \rightarrow 0
$$


induces an injection $A(T) / \ell^{n} A(T) \hookrightarrow H_{\text {ét }}^{1}\left(T, A\left[\ell^{n}\right]\right)$. Since $T$ is Noetherian, 4, Thm. 1.1, p. 233] implies that $H^{0}\left(T, A\left[\ell^{n}\right]\right)$ is finite for each $n \geq 1$. This implies that $\lim ^{1} H^{0}\left(T, A\left[\ell^{n}\right]\right)$ vanishes and that the natural surjection

$$
H_{\text {ét }}^{1}\left(T,{\underset{n}{n}}_{\lim _{n}} A\left[\ell^{n}\right]\right) \rightarrow \underset{\varliminf_{n}}{\lim _{\text {ét }}} H^{1}\left(T, A\left[\ell^{n}\right]\right)
$$

is an isomorphism. The result now follows as the Weil pairing induces an isomorphism $R^{1} \pi_{*} \mathbb{Z}_{\ell}(1) \rightarrow \lim _{n} A\left[\ell^{n}\right]$.

Corollary 12.3. If the group $A(T)$ of sections of $\pi: A \rightarrow T$ is finitely generated, then the kernel of

$$
A(T) \rightarrow H_{\text {ét }}^{1}\left(T, R^{1} \pi_{*} \mathbb{Q}_{\ell}(1)\right)
$$

is finite for all $\ell$.

The assumption that $A(T)$ be finitely generated is necessary, as can be seen by considering the case where $T$ is the spectrum of an algebraically closed field.

We can apply this to the family of Jacobians $\pi: \mathrm{Jac}_{C / T} \rightarrow T$ associated to the family of curves $C \rightarrow T$.

Corollary 12.4. Suppose that $x$ and $y$ are sections of $C$ over $T$. If $\operatorname{Jac}_{C / T}(T)$ is finitely generated, then $\kappa_{x}=\kappa_{y}$ implies that $x-y$ is torsion in $\operatorname{Jac}_{C / T}(T)$.

Proof. Since $R^{1} \pi_{*} \mathbb{Z}_{\ell}(1)$ is naturally isomorphic to $\mathbb{H}_{\mathbb{Z}_{\ell}}$, the diagram

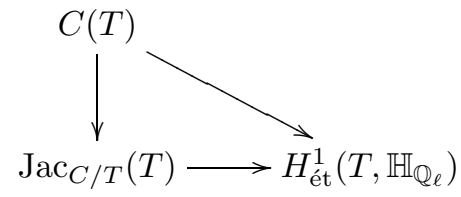

commutes, where the left-hand vertical map takes $x$ to the class of $(2 g-2) x-\omega_{C / T}$ whose image under the horizontal map is $\kappa_{x}$. Since the bottom map is injective mod torsion, $\kappa_{x}=\kappa_{y}$ implies that the divisor $(2 g-2)(x-y)$ is torsion.

We conclude this section by relating the class $\kappa_{x}$ of a section of $C \rightarrow T$ to the structure of $H_{1}\left(\mathfrak{u}_{T}\right)$. Proposition 6.8 implies that there is an isomorphism

$$
H_{\text {ét }}^{1}(T, \mathbb{H}) \cong \operatorname{Hom}_{\mathrm{GSp}(H)}\left(H_{1}\left(\mathfrak{u}_{T}\right), \mathbb{H}\right) \text {. }
$$

The GSp $(H)$-equivariant projection $h: \Lambda_{1}^{3} H \rightarrow H$ is the twist of the map $\Lambda^{3} H \rightarrow$ $H(1)$ defined by $x \wedge y \wedge z \mapsto \theta(x, y) z+\theta(y, z) x+\theta(z, x) y$.

The following assertion follows from 22 , Prop. 6.5] and its proof.

Proposition 12.5. If $g \geq 3$, the $\operatorname{GSp}(H)$-invariant homomorphism

$$
H_{1}\left(\mathfrak{u}_{g, 1}\right) \rightarrow \mathrm{Gr}_{-1}^{W} \mathfrak{u}_{g, 1} \cong \Lambda_{1}^{3} H \stackrel{2 h}{\rightarrow} H
$$

corresponds to the universal class $\kappa$ under the isomorphism

$$
H_{\text {ét }}^{1}\left(\mathcal{M}_{g, 1 / k}, \mathbb{H}_{\mathbb{Q}_{\ell}}\right) \cong \operatorname{Hom}_{\mathrm{GSp}(H)}\left(H_{1}\left(\mathfrak{u}_{g, 1}\right), H\right) .
$$

Let $h_{j}: \Lambda_{n}^{3} H \rightarrow H$ be the $\operatorname{GSp}(H)$-invariant homomorphism

$$
\Lambda_{n}^{3} H \longrightarrow\left(\Lambda_{1}^{3} H\right)^{n} \stackrel{p r_{j}}{\longrightarrow} \Lambda_{1}^{3} H \stackrel{h}{\longrightarrow} H .
$$


Corollary 12.6. When $g \geq 3$ and $n \geq 1$, the class $\kappa_{j} \in H_{\text {ét }}^{1}\left(\mathcal{M}_{g, n / k}, \mathbb{H}_{\mathbb{Q}_{\ell}}\right)$ corresponds to the homomorphism $2 h_{j}$ under the isomorphism

$$
H_{\text {ét }}^{1}\left(\mathcal{M}_{g, n / k}, \mathbb{H}_{\mathbb{Q}_{\ell}}\right) \cong \operatorname{Hom}_{\mathrm{GSp}(H)}\left(H_{1}\left(\mathfrak{u}_{g, n}\right), H\right) \text {. }
$$

Recall from the proof of Lemma 9.4 that the composite $h \circ\left(\_\wedge \check{\theta}\right): H \rightarrow \Lambda_{1}^{3} H \rightarrow H$ is $(g-1) \mathrm{id}_{H}$. It follows that the projection $\Lambda_{n}^{3} H \rightarrow H$ that corresponds to the projection

$$
\Lambda_{n}^{3} H=\Lambda_{0}^{3} H \oplus H^{n} \rightarrow H
$$

onto the $j$ th copy of $H$ is $h_{j} /(g-1)$. This projection corresponds to $\kappa_{j} /(2 g-2)$.

Remark 12.7. One of the issues in trying to prove the section conjecture is to determine which classes in $\operatorname{Pic}^{1} C$ are the classes of rational points. To provide a context for the results of the next section, we mention that when $C$ is the pullback of the universal curve to $\operatorname{Spec} k\left(\mathcal{M}_{g, n / k}[m]\right)$,

$$
\left(\operatorname{Pic}^{0} C\right)\left(k\left(\mathcal{M}_{g, n / k}[m]\right)\right) \cong \bigoplus_{j=1}^{n} \mathbb{Z}\left(\kappa_{j} / \epsilon_{m}\right) \oplus \bigoplus_{1 \leq j<j^{\prime} \leq n} \mathbb{Z}\left[x_{j}-x_{j^{\prime}}\right] \oplus(\mathbb{Z} / m \mathbb{Z})^{2 g},
$$

where $\epsilon_{m}$ is 2 when $m$ is even and 1 when $m$ is odd 14 This is proved in 14, Thm. 12.3] when $k=\mathbb{C}$; it implies the result for all algebraically closed fields $k$ of characteristic zero. The case for a general field $k$ of characteristic zero follows as all the generators are represented by $\mathbb{Q}$-rational divisors.

\section{Computation of $C(k(T))$}

Given a family of curves $C \rightarrow T$, one encounters the problem of distinguishing the sections of $\operatorname{Pic}^{1} C \rightarrow T$ that correspond to $k(T)$-rational points of $C$ from those that do not. This distinction can be made when $C / T$ is topologically ample.

Lemma 13.1. If $g \geq 3$ and $n \geq 1$, there is no $\operatorname{GSp}(H)$-equivariant Lie algebra homomorphism

$$
\mathrm{Gr}_{\bullet}^{W} \mathfrak{u}_{g, n}^{\text {geom }} / W_{-3} \rightarrow \mathrm{Gr}_{\bullet}^{W} \mathfrak{u}_{g, 2}^{\text {geom }} / W_{-3}
$$

that induces the map $\left(v ; u_{1}, \ldots, u_{n}\right) \mapsto\left(v ; u_{1}, u_{1}\right)$ on $\operatorname{Gr}_{-1}^{W}$.

Proof. Suppose that such a homomorphism $\phi: \mathrm{Gr}_{\bullet}^{W} \mathfrak{u}_{g, n}^{\text {geom }} / W_{-3} \rightarrow \mathrm{Gr}_{\bullet}^{W} \mathfrak{u}_{g, 2}^{\text {geom }} / W_{-3}$ exists. Denote the $i j$ th copy $(1 \leq i<j \leq n)$ of $\mathbb{Q}_{\ell}(1)$ in $\operatorname{Gr}_{-2}^{W} \mathfrak{u}_{g, n}^{\text {geom }}$ by $\mathbb{Q}_{\ell}(1)_{i j}$. Note that there is a unique copy of $\mathbb{Q}_{\ell}(1)$ in $\mathrm{Gr}_{-2}^{W} \mathfrak{u}_{g, 2}^{\text {geom }}$. Denote the $j$ th copy of $H$ in $\operatorname{Gr}_{-1}^{W} \mathfrak{u}_{g, n}^{\text {geom }}$ by $H_{j}$. Proposition 9.13 implies that

$$
\Lambda^{2} \Lambda_{0}^{3} H \stackrel{\text { inclusion }}{\longrightarrow} \Lambda^{2} \mathrm{Gr}_{-1}^{W} \mathfrak{u}_{g, 2}^{\text {geom }} \stackrel{\text { bracket }}{\longrightarrow} \mathrm{Gr}_{-2}^{W} \mathfrak{u}_{g, 2}^{\text {geom }} \stackrel{q_{12}}{\longrightarrow} \mathbb{Q}_{\ell}(1)
$$

is surjective. When $n=1$, there is no copy of $\mathbb{Q}_{\ell}(1)$ in $\operatorname{Gr}_{-2}^{W} \mathfrak{u}_{g, n}$, from which it follows that there is no such homomorphism $\phi$ when $n=1$.

Now suppose that $n>1$. In this case Proposition 9.13 implies that the image of

$$
\Lambda^{2} \Lambda_{0}^{3} H \stackrel{\text { inclusion }}{\longrightarrow} \Lambda^{2} \mathrm{Gr}_{-1}^{W} \mathfrak{u}_{g, n}^{\text {geom }} \stackrel{\text { bracket }}{\longrightarrow} \mathrm{Gr}_{-2}^{W} \mathfrak{u}_{g, n}^{\text {geom }} \stackrel{\oplus q_{i j}}{\longrightarrow} \bigoplus_{i<j} \mathbb{Q}_{\ell}(1)_{i j}
$$

\footnotetext{
${ }^{14}$ More precisely, when $m$ is even the relative canonical bundle has $2^{2 g}$ square roots ("theta characteristics"), any two of which differ by a point of order 2 . By $\kappa_{j} / 2$ we mean the class of $(g-1) x_{j}-\alpha$ for a choice of theta characteristic $\alpha$. This is well defined mod 2 -torsion.
} 
is the diagonal copy of $\mathbb{Q}_{\ell}(1)$. It follows that $\phi$ induces a surjective homomorphism

$$
\left(\mathrm{Gr}_{-2}^{W} \mathfrak{u}_{g, n}^{\text {geom }}\right)^{\mathrm{Sp}(H)} \rightarrow\left(\mathrm{Gr}_{-2}^{W} \mathfrak{u}_{g, 2}^{\text {geom }}\right)^{\mathrm{Sp}(H)}
$$

On the other hand, Proposition 9.13 implies that, when $i<j$, the component

$$
H_{i} \otimes H_{j} \stackrel{\text { inclusion }}{\longrightarrow} \Lambda^{2} \mathrm{Gr}_{-1}^{W} \mathfrak{u}_{g, n}^{\text {geom }} \stackrel{\text { bracket }}{\longrightarrow} \mathrm{Gr}_{-2}^{W} \mathfrak{u}_{g, n} \stackrel{q_{i j}}{\longrightarrow} \mathbb{Q}_{\ell}(1)_{i j}
$$

of the bracket is surjective. Since $j>1, H_{j}$ is contained in the kernel of $\phi$. These two facts imply that the restriction of $\phi$ to $\mathbb{Q}_{\ell}(1)_{i j}$ is zero whenever $1 \leq i<j \leq$ $n$, which contradicts the non-triviality of (15). It follows that there is no such homomorphism $\phi$ when $n>1$, which completes the proof.

Topologically ample curves have the nice property that their $T$-rational points are determined by their classes in $H^{1}\left(T, \mathbb{H}_{\mathbb{Q}_{\ell}}\right)$.

Proposition 13.2. Suppose that $C / T$ is a topologically ample curve of type $(g, n)$ where $g \geq 3$ and $n \geq 1$. If $x \in C(k(T))$ and $\kappa_{x}=\kappa_{j}$, then $x$ is the jth tautological point $x_{j}$.

Proof. We may assume that $j=1$. Corollary 12.4 implies that $[x]-\left[x_{1}\right]$ is torsion in $\left(\operatorname{Pic}^{0} C\right)(k(T))$. Denote this torsion point by $\delta$. If $\delta=0$, then $x=x_{1}$ and we are done. By replacing $T$ by $T-Z$ if necessary, where $Z$ is a subvariety of $T$ of codimension $\geq 2$, results of Section 3 imply that we may assume that $x \in C(T)$. If $\delta \neq 0$, then the sections $x$ and $x_{1}$ of $C$ are disjoint, and there is a $k$-morphism $T \rightarrow \mathcal{M}_{g, 2}$ defined by $x \mapsto\left[C_{x} ; x_{1}, x\right]$. This induces a $\operatorname{GSp}(H)$ equivariant homomorphism $\phi: \mathrm{Gr}_{\bullet}^{W} \mathfrak{u}_{T} \rightarrow \mathrm{Gr}_{\bullet}^{W} \mathfrak{u}_{g, 2}$. Since $C / T$ is topologically ample, the marking induces an identification $\mathfrak{u}_{T} / W_{-3} \cong \mathfrak{u}_{g, n} / W_{-3}$. Since $\kappa_{x}=\kappa_{1}$, the induced mapping

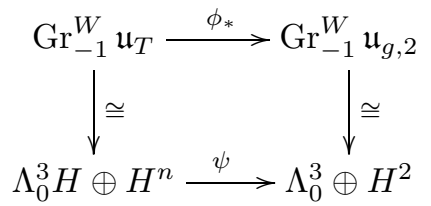

on $\mathrm{Gr}_{-1}^{W}$ is defined by $\psi:\left(v ; u_{1}, \ldots, u_{n}\right) \mapsto\left(v ; u_{1}, u_{1}\right)$. But by Lemma 13.1, there is no such homomorphism. It follows that $\delta=0$ and that $x=x_{1}$.

13.1. Proof of Theorem 1. If $C(k(T))$ is empty, $n$ must be zero and the result is true. Suppose that $x \in C(k(T))$. Corollary 3.2 implies that $x$ induces a splitting of the homomorphism $\pi_{1}\left(C, \bar{\eta}_{C}\right) \rightarrow \pi_{1}\left(T, \bar{\eta}_{T}\right)$ for some choice of basepoints. Corollary 11.6 implies that $x$ induces a $\operatorname{GSp}(H)$-equivariant splitting of $\mathfrak{d}\left(\mathfrak{u}_{C}\right) \rightarrow \mathfrak{d}\left(\mathfrak{u}_{T}\right)$ which, when $g=3$, preserves the $\mathbb{Z}_{2}$ lattice structure on $\mathrm{Gr}_{-1}^{W}$. Proposition 10.8 implies that $n \geq 1$ and that this section equals the section induced by the tautological point $x_{j}$ for some $j \in\{1, \ldots, n\}$. Corollary 12.6 implies that $\kappa_{x}=\kappa_{j}$. Proposition 13.2 and Remark 3.3 now imply that $x=x_{j}$.

\section{NON-ABELIAN COHOMOLOGY}

This section is a brief review of the non-abelian cohomology of proalgebraic groups developed in [19], which was inspired by, and is a variant of, the non-abelian cohomology developed by Kim in 28. This non-abelian cohomology is the principal tool used in the proof of Theorem 3 This version of non-abelian cohomology should 
be useful in studying rational points of curves and other non-abelian varieties as it is somewhat computable, as we demonstrate in Section 18 .

Suppose that $F$ is a field of characteristic zero and that $\mathcal{G}$ is a negatively weighted extension of a connected, reductive $F$-group $R$ with respect to a non-trivial central cocharacter $\omega: \mathbb{G}_{m} \rightarrow R$. Suppose that $\mathcal{P}$ is a prounipotent $F$-group with trivial center. Fix an outer action $\phi: \mathcal{G} \rightarrow$ Out $\mathcal{P}$ of $\mathcal{G}$ on $\mathcal{P}$. This induces an action of $\mathcal{G}$ on $H_{1}(\mathcal{P})$, so that $H_{1}(\mathcal{P})$ has a natural weight filtration. We will assume that this is negatively weighted, i.e., $H_{1}(\mathcal{P})=W_{-1} H_{1}(\mathcal{P})$, and that each of its weight graded quotients is finite dimensional. Under these conditions, $\mathcal{P}$ has a natural weight filtration with finite dimensional quotients and the group of weight filtration preserving automorphisms $\mathrm{Aut}_{W} \mathcal{P}$ of $\mathcal{P}$ is a proalgebraic group. We will define a non-abelian cohomology scheme $H_{\text {nab }}^{1}(\mathcal{G}, \mathcal{P})$, which will represent sections of an extension of $\mathcal{G}$ by $\mathcal{P}$.

The condition that $\mathcal{P}$ have trivial center implies that this extension is uniquely determined by the outer action $\phi$. Indeed, since $\mathcal{P}$ has trivial center, the sequence

$$
1 \rightarrow \mathcal{P} \rightarrow \text { Aut }_{W} \mathcal{P} \rightarrow \mathrm{Out}_{W} \mathcal{P} \rightarrow 1,
$$

where the first map takes an element of $\mathcal{P}$ to the corresponding inner automorphism, is an exact sequence of proalgebraic $F$-groups. Pulling this extension back along $\phi$ gives an extension

$$
1 \rightarrow \mathcal{P} \rightarrow \widehat{\mathcal{G}}_{\phi} \rightarrow \mathcal{G} \rightarrow 1
$$

of $\mathcal{G}$ by $\mathcal{P}$. We do not make the conventional assumption that this sequence splits, nor do we distinguish any one section when there is one.

The assumption that $H_{1}(\mathcal{P})$ be negatively weighted implies that $\widehat{\mathcal{G}}_{\phi}$ is also a negatively weighted extension of $R$ with respect to $\omega$. It also implies that $W_{-m} \mathcal{P}$ is a normal subgroup of $\widehat{\mathcal{G}}_{\phi}$ for all $m \in \mathbb{Z}$.

Denote the Lie algebra of $\mathcal{G}$ by $\mathfrak{g}$. It acts on the weight graded quotients of $\mathfrak{p}$. Frequently we will impose the finiteness condition

$$
\operatorname{dim} H^{1}\left(\mathfrak{g}, \operatorname{Gr}_{-m}^{W} \mathfrak{p}\right)<\infty
$$

for $m \in \mathbb{Z}$ in a suitable range. The following is our analogue of [28, Prop. 2].

Theorem 14.1. Suppose that $N>1$ and that the finiteness condition (17) holds when $1 \leq m<N$. Under the assumptions above, there is an affine F-scheme of finite type, $H_{\text {nab }}^{1}\left(\mathcal{G}, \mathcal{P} / W_{-N} \mathcal{P}\right)$, which represents the functor that takes an $F$-algebra A to the set

$$
\left\{\text { sections of }\left(\widehat{\mathcal{G}}_{\phi} / W_{-N} \mathcal{P}\right) \otimes_{F} A \rightarrow \mathcal{G} \otimes_{F} A\right\} / \text { conjugation by } \mathcal{P}(A),
$$

where $\mathcal{P}(A)$ acts on the sections by conjugation.

Corollary 14.2. If the finiteness condition (17) holds for all $m \geq 1$, then the affine F-scheme

$$
H_{\text {nab }}^{1}(\mathcal{G}, \mathcal{P}):=\lim _{\longleftarrow} H_{\text {nab }}^{1}\left(\mathcal{G}, \mathcal{P} / W_{-N} \mathcal{P}\right)
$$

represents the functor

$$
\left\{\text { sections of } \widehat{\mathcal{G}}_{\phi} \otimes_{F} A \rightarrow \mathcal{G} \otimes_{F} A\right\} / \text { conjugation by } \mathcal{P}(A) .
$$

There is a non-abelian analogue of an exact sequence which aids in the computation of $H_{\text {nab }}^{1}(\mathcal{G}, \mathcal{P})$. The next result is our analogue of the claim on page 641 of 28. 
Theorem 14.3. Suppose that $N>1$. If the finiteness condition (17) is satisfied when $1 \leq m \leq N$, then

(i) there is a morphism $\delta: H_{\text {nab }}^{1}\left(\mathcal{G}, \mathcal{P} / W_{-N}\right) \rightarrow H^{2}\left(\mathfrak{g}, \operatorname{Gr}_{-N}^{W} \mathfrak{p}\right)$ of $F$-schemes,

(ii) there is a principal action of $H^{1}\left(\mathfrak{g}, \mathrm{Gr}_{-N}^{W} \mathfrak{p}\right)$ on $H_{\text {nab }}^{1}\left(\mathcal{G}, \mathcal{P} / W_{-N-1}\right)$,

(iii) for all $F$-algebras $A, H_{\text {nab }}^{1}\left(\mathcal{G}, \mathcal{P} / W_{-N}\right)(A)$ is a principal $H^{1}\left(\mathfrak{g}, \mathrm{Gr}_{-N}^{W} \mathfrak{p}\right)(A)$ set over the $A$-rational points $\left(\delta^{-1}(0)\right)(A)$ of the scheme $\delta^{-1}(0)$.

This "exact sequence" is represented by the diagram:

$$
H^{1}\left(\mathfrak{g}, \operatorname{Gr}_{-N}^{W} \mathfrak{p}\right) \subseteq H_{\text {nab }}^{1}\left(\mathcal{G}, \mathcal{P} / W_{-N-1}\right) \stackrel{\pi}{\longrightarrow} H_{\text {nab }}^{1}\left(\mathcal{G}, \mathcal{P} / W_{-N}\right) \stackrel{\delta}{\longrightarrow} H^{2}\left(\mathfrak{g}, \operatorname{Gr}_{-N}^{W} \mathfrak{p}\right)
$$

in which $\pi$ is the projection that takes a section to its quotient by $\operatorname{Gr}_{-N}^{W} \mathcal{P}$.

Remark 14.4. In our version of non-abelian cohomology, we do not need to specify a splitting of the extension (16), or even to have one at all. Since this extension may not split, $H_{\text {nab }}^{1}(\mathcal{G}, \mathcal{P})$ may be the empty scheme. Even when the extension does split, there may be no preferred section and we do not view these cohomology groups as pointed objects, as is customary. This point of view is based on the computations in Section [18, which suggest that preferring any one section over another may be unnatural in some contexts.

14.1. The scheme $H_{\text {nab }}^{1}\left(\mathcal{G}, \mathcal{P} / W_{-N-1}\right)$. In Section 18, we will need to compute the "connecting morphism" $\delta$ in situations of interest. Before we explain how to compute $\delta$, we need to explain why $H_{\text {nab }}^{1}\left(\mathcal{G}, \mathcal{P} / W_{-m-1} \mathcal{P}\right)$ is a scheme. Full details can be found in 19 .

Denote $\widehat{\mathcal{G}}_{\phi}$ by $\widehat{\mathcal{G}}$. For each $m>0$, set $\widehat{\mathcal{G}}_{m}=\widehat{\mathcal{G}} / W_{-m-1} \mathcal{P}$. Denote the Lie algebras of $\mathcal{G}, \widehat{\mathcal{G}}, \widehat{\mathcal{G}}_{m}, \mathcal{P}$ and $\mathcal{P} / W_{-m-1}$ by $\mathfrak{g}, \hat{\mathfrak{g}}, \hat{\mathfrak{g}}_{m}, \mathfrak{p}$ and $\mathfrak{p}_{m}$, respectively. Choose a lift $\hat{\omega}: \mathbb{G}_{m} \rightarrow \widehat{\mathcal{G}}$ of $\omega$ to $\widehat{\mathcal{G}}$. Denote its composition with $\widehat{\mathcal{G}} \rightarrow \widehat{\mathcal{G}}_{m}$ by $\hat{\omega}_{m}$ and with $\widehat{\mathcal{G}} \rightarrow \mathcal{G}$ by $\hat{\omega}_{0}$. Note that $\hat{\omega}$ splits the weight filtration of all $\widehat{\mathcal{G}}$-modules. In particular, it splits the weight filtration of each of these Lie algebras.

Fix $N \geq 1$. Let $A$ be an $F$-algebra. Define a section $s$ of $\widehat{\mathcal{G}}_{N} \otimes_{F} A \rightarrow \mathcal{G} \otimes_{F} A$ to be $\hat{\omega}$-graded if it commutes with $\hat{\omega}$ in the sense that $\hat{\omega}_{N}=s \circ \hat{\omega}_{0}$. Equivalently, its derivative $d s: \mathfrak{g} \otimes_{F} A \rightarrow \hat{\mathfrak{g}} \otimes_{F} A$ is $\hat{\omega}$-graded in the sense that it commutes with the $\mathbb{G}_{m}$-actions on $\mathfrak{g}$ and $\hat{\mathfrak{g}}$ induced by $\hat{\omega}$.

One has the maps

$$
\begin{aligned}
& \left\{\hat{\omega} \text {-graded sections of } \hat{\mathfrak{g}}_{N} \otimes_{F} A \rightarrow \mathfrak{g} \otimes_{F} A\right\} \\
& \cong\left\{\hat{\omega} \text {-graded sections of } \widehat{\mathcal{G}}_{N} \otimes_{F} A \rightarrow \mathcal{G} \otimes_{F} A\right\} \\
& \quad \hookrightarrow\left\{\text { sections of } \widehat{\mathcal{G}}_{N} \otimes_{F} A \rightarrow \mathcal{G} \otimes_{F} A\right\} \\
& \left.\quad \stackrel{\mathrm{Gr}^{W}}{\rightarrow} \text { \{graded sections of } \mathrm{Gr}_{\bullet}^{W} \hat{\mathfrak{g}}_{N} \otimes_{F} A \rightarrow \mathrm{Gr}_{\bullet}^{W} \mathfrak{g} \otimes_{F} A\right\},
\end{aligned}
$$

where the last map takes a section $s$ to the graded Lie algebra section induced by its derivative $d s$. Note that the composition of these three maps is the isomorphism induced by the $\hat{\omega}$-splitting of the weight filtration.

It is not difficult to show (cf. [19, Prop. 4.3]) that if the finiteness assumption (17) holds for all $m \leq N$, then the functor that takes an $F$-algebra $A$ to the set

$$
\text { \{graded sections of } \mathrm{Gr}_{\bullet}^{W} \hat{\mathfrak{g}}_{N} \otimes_{F} A \rightarrow \mathrm{Gr}_{\bullet}^{W} \mathfrak{g} \otimes_{F} A \text { \} }
$$


is represented by an affine $F$-scheme of finite type, which we shall denote by Sect. $\left(\mathrm{Gr}_{\bullet}^{W} \mathfrak{g}, \mathrm{Gr}_{\bullet}^{W} \mathfrak{p} / W_{-N-1}\right)$. It is a subscheme of an affine space that is a principal homogeneous space over $\operatorname{Hom}_{R}\left(\mathrm{Gr}_{\bullet}^{W} H_{1}(\mathfrak{g}), \mathrm{Gr}_{\bullet}^{W} \mathfrak{p}_{N}\right)$. The finiteness assumption implies that this is finite dimensional.

Proposition 14.5 ([19, Cor. 4.7]). If $N \geq 0$, then, for all $F$-algebras $A$ the maps (18) induce bijections

$$
\begin{aligned}
\left\{\hat{\omega} \text {-graded sections of } \hat{\mathfrak{g}}_{N} \otimes_{F} A \rightarrow \mathfrak{g} \otimes_{F} A\right\} & \\
\stackrel{\simeq}{\rightarrow} & \text { sections of } \left.\widehat{\mathcal{G}}_{N} \otimes_{F} A \rightarrow \mathcal{G} \otimes_{F} A\right\} / \mathcal{P}(A) \\
& \stackrel{\mathrm{Gr}^{W}}{\rightarrow}\left\{\text { graded sections of } \mathrm{Gr}_{\bullet}^{W} \hat{\mathfrak{g}}_{N} \otimes_{F} A \rightarrow \mathrm{Gr}_{\bullet}^{W} \mathfrak{g} \otimes_{F} A\right\} .
\end{aligned}
$$

This follows from the observation [19, Lem. 4.2] that each $\mathcal{P}(A)$-conjugacy class of sections contains a unique $\hat{\omega}_{N}$-graded section. It follows that the functor that takes $A$ to the set of $\mathcal{P}(A)$ conjugacy classes of sections of $\widehat{\mathcal{G}}_{N} \otimes_{F} A \rightarrow$ $\mathcal{G} \otimes_{F} A$ is represented by the affine $F$-scheme $\operatorname{Sect} \bullet\left(\operatorname{Gr}_{\bullet}^{W} \mathfrak{g}, \operatorname{Gr}_{\bullet}^{W} \mathfrak{p} / W_{-N-1}\right)$. That is, $H_{\text {nab }}^{1}\left(\mathcal{G}, \mathcal{P} / W_{-N-1} \mathcal{P}\right)=\operatorname{Sect} \bullet\left(\operatorname{Gr}_{\bullet}^{W} \mathfrak{g}, \operatorname{Gr}_{\bullet}^{W} \mathfrak{p} / W_{-N-1}\right)$.

14.2. The connecting morphism $\delta$. Full details of the construction of $\delta$ and its properties can be found in 19 .

Suppose that $m \geq 0$. Identify $H_{\text {nab }}^{1}\left(\mathcal{G}, \mathcal{P}_{m}\right)$ with the scheme of graded sections of $\mathrm{Gr}_{\bullet}^{W} \hat{\mathfrak{g}}_{m} \rightarrow \mathrm{Gr}_{\bullet}^{W} \mathfrak{g}_{\bullet}$. Suppose that $N \geq 1$. Set $\mathfrak{z}=\mathrm{Gr}_{-N}^{W} \mathfrak{p}$. Then we have the extension of graded Lie algebras

$$
0 \rightarrow \mathfrak{z} \rightarrow \mathrm{Gr}_{\bullet}^{W} \mathfrak{g}_{N} \rightarrow \mathrm{Gr}_{\bullet}^{W} \hat{\mathfrak{g}}_{N-1} \rightarrow 0 .
$$

Suppose that $\sigma$ is a graded section of $\mathrm{Gr}_{\bullet}^{W} \hat{\mathfrak{g}}_{N-1} \rightarrow \mathrm{Gr}_{\bullet}^{W} \mathfrak{g}$. Define $\tilde{\sigma}$ to be the $R$-linear section

$$
\mathrm{Gr}_{\bullet}^{W} \mathfrak{g} \otimes_{F} A \stackrel{\sigma}{\longrightarrow} \mathrm{Gr}_{\bullet}^{W} \mathfrak{g}_{N-1} \otimes_{F} A \stackrel{r \otimes A}{\longrightarrow} \mathrm{Gr}_{\bullet}^{W} \hat{\mathfrak{g}}_{N} \otimes_{F} A
$$

of $\mathrm{Gr}_{\bullet}^{W} \hat{\mathfrak{g}}_{N} \otimes_{F} A \rightarrow \mathrm{Gr}_{\bullet}^{W} \mathfrak{g} \otimes_{F} A$, where $r$ is the unique, $R$-linear, graded section of $\mathrm{Gr}_{\bullet}^{W} \hat{\mathfrak{g}}_{N} \rightarrow \mathrm{Gr}^{W} \hat{\mathfrak{g}}_{N-1}$. Note that, in general, $r$ is not a Lie algebra homomorphism. The obstruction to $\tilde{\sigma}$ being a Lie algebra homomorphism is the function $h_{\sigma}: \Lambda^{2} \mathrm{Gr}_{\bullet}^{W} \mathfrak{g}_{N} \rightarrow \mathfrak{z}$ defined by

$$
h_{\sigma}(x \wedge y):=[\tilde{\sigma}(x), \tilde{\sigma}(y)]-\tilde{\sigma}([x, y]) .
$$

It is an $R$-invariant 2-cocycle of weight 0 in the Chevalley-Eilenberg cochain complex $C^{\bullet}\left(\mathrm{Gr}_{\bullet}^{W} \mathfrak{g}, \mathfrak{z}\right) \otimes_{F} A$. Define

$$
\delta: H_{\text {nab }}^{1}\left(\mathcal{G}, \mathcal{P}_{N}\right)(A) \rightarrow H^{2}(\mathfrak{g}, \mathfrak{z}) \otimes_{F} A
$$

by taking $\sigma$ to the class of $h_{\sigma}$ in $H^{2}\left(\mathrm{Gr}_{\bullet}^{W} \mathfrak{g}, \mathfrak{z}\right) \otimes_{F} A \cong H^{2}(\mathfrak{g}, \mathfrak{z}) \otimes_{F} A$. This is induced by a morphism of $F$-schemes.

If $\delta(\sigma) \neq 0, \sigma$ does not lift to a graded Lie algebra section of $\mathrm{Gr}_{\bullet}^{W} \hat{\mathfrak{g}}_{N} \otimes_{F} A \rightarrow$ $\mathfrak{g} \otimes_{F} A$. If $\delta(\sigma)=0$, then, by [19, Lem. 3.3], there is an $R$-invariant 1-cochain

$$
v \in \mathrm{Gr}_{0}^{W} C^{1}\left(\mathrm{Gr}_{\bullet}^{W} \mathfrak{g}, \mathfrak{z}\right) \otimes_{F} A
$$

such that $\delta(v)=h_{\delta}$. Then $\tilde{\sigma}+v: \operatorname{Gr}_{\bullet}^{W} \mathfrak{g} \otimes_{F} A \rightarrow \operatorname{Gr}_{\bullet}^{W} \hat{\mathfrak{g}}_{N} \otimes_{F} A$ is a graded Lie algebra section of $\mathrm{Gr}_{\bullet}^{W} \hat{\mathfrak{g}}_{N} \otimes_{F} A \rightarrow \mathrm{Gr}_{\bullet}^{W} \mathfrak{g} \otimes_{F} A$ that lifts $\sigma$. 


\section{Setup for proofs of Theorems 2 AND 3}

The following three sections are devoted to the proofs of Theorems 2 and 3 . This section sets up the notation to be used in these sections and establishes some basic results.

Suppose that $g, n$ and $m$ are non-negative integers satisfying $g \geq 2, n \geq 0$ and $m \geq 115$ Suppose that $k$ is a field of characteristic zero that contains $\boldsymbol{\mu}_{m}(\bar{k})$, the $m$ th roots of unity. Fix a primitive $m$ th root of unity. We then have the geometrically connected moduli space $\mathcal{M}_{g, n / k}[m]$.

Set $K=k\left(\mathcal{M}_{g, n}[m]\right)$. Fix an algebraic closure $\bar{K}$ of $K$. Denote the algebraic closure of $k$ in $\bar{K}$ by $\bar{k}$. Set

$$
G_{k}=\operatorname{Gal}(\bar{k} / k), \quad G_{K}=\operatorname{Gal}(\bar{K} / k), \text { and } G_{K}^{\text {geom }}:=\operatorname{Gal}(\bar{K} / \bar{k}) .
$$

There is an exact sequence

$$
1 \rightarrow G_{K}^{\text {geom }} \rightarrow G_{K} \rightarrow G_{k} \rightarrow 1
$$

Let $C$ be the restriction of the universal (complete) curve $\mathcal{C} \rightarrow \mathcal{M}_{g, n / k}[m]$ to its generic point Spec $K$. Choose a geometric point $\bar{x}$ of $C \otimes_{K} \bar{K}$ that lies over its generic point. Set

$$
\pi=\pi_{1}\left(C \otimes_{K} \bar{K}, \bar{x}\right)
$$

Then one has the extension

$$
1 \rightarrow \pi \rightarrow \pi_{1}(C, \bar{x}) \rightarrow G_{K} \rightarrow 1
$$

Suppose that $\ell$ is a prime number. Set

$$
H=H_{\mathbb{Q}_{\ell}}=H_{\text {ét }}^{1}\left(C \otimes_{K} \bar{K}, \mathbb{Q}_{\ell}(1)\right) .
$$

The monodromy representation $\rho: G_{K} \rightarrow \operatorname{GSp}(H)$ has Zariski dense image if and only if the image of the $\ell$-adic cyclotomic character $\chi_{\ell}: G_{k} \rightarrow \mathbb{Z}_{\ell}^{\times}$is infinite. Suppose that this is the case. Define the weight of a representation $G_{K} \rightarrow \operatorname{GSp}(H) \rightarrow$ Aut $V$ to be its weight as a $\operatorname{GSp}(H)$-module. With this convention, $H$ is a $G_{K^{-}}$ module of weight -1 .

Denote the weighted completions of $G_{K}$ with respect to $\rho$ by $\mathcal{G}_{K}$ and the weighted completion of $\pi_{1}(C, \bar{\eta})$ with respect to $\pi_{1}(C, \bar{\eta}) \rightarrow G_{K} \rightarrow \operatorname{GSp}(H)$ by $\mathcal{G}_{C}$. Denote their Lie algebras by $\mathfrak{g}_{K}$ and $\mathfrak{g}_{C}$, respectively. Denote their prounipotent radicals by $\mathcal{U}_{K}$ and $\mathcal{U}_{C}$, and their Lie algebras by $\mathfrak{u}_{K}$ and $\mathfrak{u}_{C}$, respectively. One has the exact sequence of $\mathbb{Q}_{\ell}$-groups

$$
1 \rightarrow \pi^{\mathrm{un}} \rightarrow \mathcal{G}_{C} \rightarrow \mathcal{G}_{K} \rightarrow 1
$$

where $\pi^{\text {un }}$ denotes the continuous unipotent completion $\pi_{/ \mathbb{Q}_{\ell}}^{\text {un }}$ of over $\mathbb{Q}_{\ell}$. Denote the Lie algebra of $\pi^{\text {un }}$ by $\mathfrak{p}$. Since $\mathfrak{g}_{K}, \mathfrak{g}_{C}$ and $\mathfrak{p}$ are $\mathcal{G}_{C}$-modules via the conjugation action, all have compatible natural weight filtrations that possess the exactness properties described in Proposition 6.4.

Basic naturality properties of weighted completion imply:

Proposition 15.1. For each $\mathbb{Q}_{\ell}$-algebra $A$, there is a group extension

$$
1 \rightarrow \pi^{\mathrm{un}}(A) \rightarrow \widetilde{G}_{C}^{A} \rightarrow G_{K} \rightarrow 1
$$

\footnotetext{
${ }^{15}$ Later, we will assume that $g \geq 5$. But, for the time being, we consider smaller genera so that some of the preliminary results can be presented in their natural generality.
} 
and an inclusion

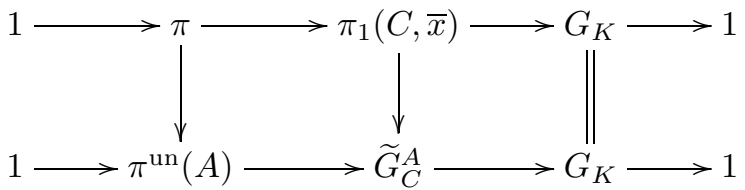

of extensions, where the left-hand vertical map is the canonical inclusion.

Proof. Define $\widetilde{G}_{C}^{A}$ so that

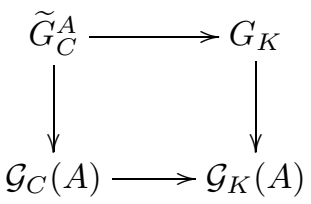

is a pullback square. The map $\widetilde{G}_{C}^{A} \rightarrow G_{K}$ is surjective because the lower map is surjective. To see this, choose a splitting of $\mathcal{G}_{C} \rightarrow \operatorname{GSp}(H)$ in the category of $\mathbb{Q}_{\ell}$-groups. It descends to a splitting of $\mathcal{G}_{K} \rightarrow \mathrm{GSp}(H)$. These splittings induce compatible isomorphisms $\mathcal{G}_{C}(A) \cong \operatorname{GSp}\left(H_{A}\right) \ltimes \mathcal{U}_{C}(A)$ and $\mathcal{G}_{K}(A) \cong \operatorname{GSp}\left(H_{A}\right) \ltimes$ $\mathcal{U}_{K}(A)$. Since $\mathcal{U}_{C} \rightarrow \mathcal{U}_{K}$ is a surjective homomorphism of prounipotent groups, the map $\mathcal{U}_{C}(A) \rightarrow \mathcal{U}_{K}(A)$ is surjective, which implies that $\mathcal{G}_{C}(A) \rightarrow \mathcal{G}_{K}(A)$ is surjective.

When $n \geq 1, \widetilde{G}_{C}^{A}$ is isomorphic to the group $G_{K} \ltimes \pi^{\text {un }}(A)$ described in the introduction.

Since $W_{-r} \pi^{\mathrm{un}}$ is the $r$ th term of its lower central series, each $W_{-r} \pi^{\mathrm{un}}$ is a normal subgroup of $\mathcal{G}_{C}$. Consequently, $W_{-r} \pi^{\text {un }}(A)$ is a normal subgroup of $\widetilde{G}_{C}^{A}$. One thus has the truncated extensions

$$
1 \rightarrow\left(\pi^{\mathrm{un}} / W_{-r} \pi^{\mathrm{un}}\right)(A) \rightarrow \widetilde{G}_{C}^{A} /\left(W_{-r} \pi^{\mathrm{un}}(A)\right) \rightarrow G_{K} \rightarrow 1
$$

for each extension field $A$ of $\mathbb{Q}_{\ell}$. Define $H_{\text {nab }}^{1}\left(G_{K}, \pi^{\text {un }} / W_{-r}\right)(A)$ to be the set of sections of $\widetilde{G}_{C}^{A} \rightarrow G_{K}$, modulo conjugation by $\pi^{\mathrm{un}}(A) / W_{-r}$.

Proposition 15.2. Suppose that $r \in[1, \infty]$. If $H^{1}\left(G_{K}, \mathrm{Gr}_{-s}^{W} \pi^{\mathrm{un}}\right)$ is finite dimensional when $l \leq s<r$, then the restriction mapping

$$
H_{\text {nab }}^{1}\left(\mathcal{G}_{K}, \pi^{\text {un }} / W_{-r}\right)\left(\mathbb{Q}_{\ell}\right) \rightarrow H_{\text {nab }}^{1}\left(G_{K}, \pi^{\text {un }} / W_{-r}\right)\left(\mathbb{Q}_{\ell}\right)
$$

is a bijection.

Conditions that ensure the finite dimensionality of $H^{1}\left(G_{K}, \mathrm{Gr}_{-r}^{W} \pi^{\mathrm{un}}\right)$ are given in Section 17. The condition is satisfied, for example, when $k$ is a number field and $g \geq 3$.

Proof. Proposition 6.8 implies that $H^{1}\left(G_{K}, \mathrm{Gr}_{-s}^{W} \pi^{\mathrm{un}}\right) \cong H^{1}\left(\mathcal{G}_{K}, \mathrm{Gr}_{-s}^{W} \pi^{\text {un }}\right)$. The finite dimensionality of $H^{1}\left(G_{K}, \mathrm{Gr}_{-s}^{W} \pi^{\text {un }}\right)$ thus ensures the existence of the scheme $H_{\text {nab }}^{1}\left(\mathcal{G}_{K}, \pi^{\text {un }} / W_{-r}\right)$. Suppose that $s$ is a section of $\widetilde{G}_{C}^{\mathbb{Q}_{\ell}} / W_{-r} \pi^{\text {un }}\left(\mathbb{Q}_{\ell}\right) \rightarrow G_{K}$. Then the universal mapping property of weighted completion implies that

$$
G_{K} \stackrel{s}{\longrightarrow} \widetilde{G}_{C}^{\mathbb{Q}_{\ell}} / W_{-r} \pi^{\mathrm{un}}\left(\mathbb{Q}_{\ell}\right) \longrightarrow\left(\mathcal{G}_{C} / W_{-r} \pi^{\mathrm{un}}\right)\left(\mathbb{Q}_{\ell}\right)
$$


induces a section $\sigma$ of $\mathcal{G}_{C} \rightarrow \mathcal{G}_{K} / W_{-r} \pi^{\mathrm{un}}\left(\mathbb{Q}_{\ell}\right)$ such that the diagram

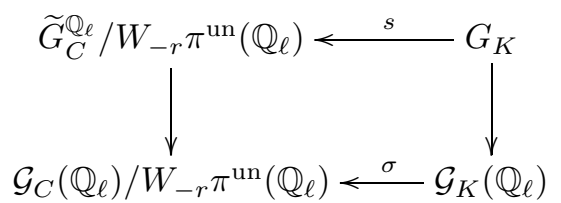

commutes. On the other hand, since (20) is a pullback square, so is its reduction $\bmod W_{-r} \pi^{\mathrm{un}}\left(\mathbb{Q}_{\ell}\right)$. Consequently, each section $\sigma$ of $\left(\mathcal{G}_{C} / W_{-r} \pi^{\mathrm{un}}\right)\left(\mathbb{Q}_{\ell}\right) \rightarrow \mathcal{G}_{K}$ induces a section $s$ of $\widetilde{G}_{C}^{\mathbb{Q}_{\ell}} / W_{-r} \pi^{\mathrm{un}}\left(\mathbb{Q}_{\ell}\right) \rightarrow G_{K}$ such that the diagram above commutes. The results follow as two sections $s_{1}$ and $s_{2}$ of $\widetilde{G}_{C}^{\mathbb{Q}_{\ell}} \rightarrow G_{K}$ are conjugate by an element of $\pi^{\mathrm{un}}\left(\mathbb{Q}_{\ell}\right)$ if and only if the corresponding sections $\sigma_{1}$ and $\sigma_{2}$ of $\mathcal{G}_{C} \rightarrow \mathcal{G}_{K}$ are conjugate by an element (necessarily the same $\bmod W_{-r} \pi^{\mathrm{un}}\left(\mathbb{Q}_{\ell}\right)$ ) of $\pi^{\text {un }}\left(\mathbb{Q}_{\ell}\right)$.

\section{Cohomology computations}

Theorems 2 and 3 are proved using the non-abelian cohomology described in Section 14. In order to compute $H_{\text {nab }}^{1}\left(\mathcal{G}_{K}, \pi^{\text {un }}\right)$, we need to compute $H^{1}\left(\mathfrak{g}_{K}, \mathrm{Gr}_{-r}^{W} \mathfrak{p}\right)$ and to bound $H^{2}\left(\mathfrak{g}_{K}, \mathrm{Gr}_{-r}^{W} \mathfrak{p}\right)$ for each $r>0$. This we do in the next section. But first we need to compute and/or bound the cohomology groups $H^{\bullet}\left(G_{K}, V\right)$ in low degrees for all negatively weighted $\operatorname{GSp}(H)$-modules $V$ as these control the cohomology groups $H^{\bullet}\left(\mathfrak{g}_{K}, \mathrm{Gr}_{-r}^{W} \mathfrak{p}\right)$ in low degree. In this section we do this by investigating the degree to which the restriction mapping

$$
H_{\text {ét }}^{\bullet}\left(\mathcal{M}_{g, n / k}[m], \mathbb{V}\right) \rightarrow H^{\bullet}\left(G_{K}, V\right)
$$

is an isomorphism. Remarkably, it is an isomorphisms in degree 1 and an injection in degree 2, except when the pullback of $\mathbb{V}$ to $\mathcal{M}_{g, n / \bar{k}}[m]$ contains a trivial local system.

Recall that $\mathcal{C}_{g / k}^{n}[m]$ denotes the $n$th power of the universal (complete) curve $\mathcal{C}_{g}$ over $\mathcal{M}_{g / k}[m]$. By convention, $\mathcal{C}_{g / k}^{0}[m]=\mathcal{M}_{g / k}[m]$. Note that $\mathcal{M}_{g, n / k}[m]$ is a Zariski open subset of $\mathcal{C}_{g / k}^{n}[m]$.

The irreducible $\operatorname{GSp}\left(H_{\mathbb{Q}_{\ell}}\right)$-module $H_{\lambda}(r)$ gives rise to a $G_{K}$-module of weight $-|\lambda|-2 r$ by composition with $\rho$. Denote the local system over $\mathcal{M}_{g, n / k}[m]$ corresponding to the $\operatorname{GSp}(H)$-module $V$ by $\mathbb{V}$. Call an irreducible $\operatorname{GSp}\left(H_{\mathbb{Q}_{\ell}}\right)$-module $V$ geometrically non-trivial if it is non-trivial when restricted to $\operatorname{Sp}\left(H_{\mathbb{Q}_{\ell}}\right)$.

The following key result follows from [18, Cor. 6.2] and standard comparison theorems.

Theorem 16.1. Suppose that $k$ is an algebraically closed field of characteristic zero. Suppose that $n \geq 0, m \geq 1$, and that $g=3$ or $g \geq 5$. If $U$ is a Zariski open subset of $\mathcal{C}_{g / k}^{n}[\mathrm{~m}]$, then for all non-trivial, irreducible, geometrically non-trivial representations $V$ of $\operatorname{GSp}(H)$, the map

$$
H_{\text {ét }}^{j}\left(\mathcal{C}_{g}^{n}[m], \mathbb{V}\right) \rightarrow H_{\text {ét }}^{j}(U, \mathbb{V})
$$

induced by the inclusion $U \hookrightarrow \mathcal{C}_{g}^{n}[m]$ is an isomorphism when $j=0,1$ and an injection when $j=2$.

Since $\mathcal{M}_{g, n / k}[m]$ is a Zariski open subset of $\mathcal{C}_{g}^{n}[m]$, the result remains true when $\mathcal{C}_{g}^{n}[m]$ is replaced by $\mathcal{M}_{g, n}[m]$ in the statement of the theorem. 
Remark 16.2. Theorem 16.1 holds in a more general situation, which is described in the following paragraph. It allows the reduction of the transcendence degree of the field $K$ in Theorem 3 from $3 g-3+n$ to 3 . The key point is that a more general version of the previous theorem implies that the cohomological computations in this section hold in this more general situation. For clarity of exposition, we do not work out the details in this more general situation, but leave them to the interested reader.

Suppose that $k$ is an algebraically closed field of characteristic zero. First let $M$ be a generic linear section of $\mathcal{M}_{g / k}[\mathrm{~m}]$ of dimension $\geq 3$. Denote the restriction of the $n$th power of the universal curve to $M$ by $C_{M}^{n}$. For a suitable imbedding $C_{M}^{n} \rightarrow \mathbb{P}^{N}$, [18, Thm. 5.1] implies (cf. [18, Thm. 6.1]) that a generic section $T$ of $C_{M}^{n}$ by a complete intersection of sufficiently high multi-degree will have the property that for all non-trivial, irreducible, geometrically non-trivial representations $V$ of $\operatorname{GSp}(H)$, and all Zariski open subsets $U$ of $T$, the map $H_{\text {ét }}^{j}(T, \mathbb{V}) \rightarrow H_{\text {ét }}^{j}(U, \mathbb{V})$ induced by the inclusion $U \hookrightarrow T$ is an isomorphism when $j=0,1$ and an injection when $j=2$.

Set $K=k\left(\mathcal{M}_{g, n / k}[m]\right)$ and let $C / K$ be the restriction of the universal curve to $\operatorname{Spec} K$.

Proposition 16.3. Suppose that $g \geq 5$ and that $k$ is a field of characteristic zero for which the image of the $\ell$-adic cyclotomic character $\chi_{\ell}: G_{k} \rightarrow \mathbb{Z}_{\ell}^{\times}$is infinite.

(i) If $r \neq 1$, then $H^{1}\left(G_{K}, \mathbb{Q}_{\ell}(r)\right) \cong H^{1}\left(G_{k}, \mathbb{Q}_{\ell}(r)\right)$.

(ii) If $V$ is a geometrically non-trivial, irreducible representation of $\operatorname{GSp}\left(H_{\mathbb{Q}_{\ell}}\right)$ of negative weight, then

$$
H^{1}\left(G_{K}, V\right) \cong H_{\text {êt }}^{1}\left(\mathcal{M}_{g, n / k}[m], \mathbb{V}\right) \cong \begin{cases}\mathbb{Q}_{\ell}{ }^{n} & V=H_{\mathbb{Q}_{\ell}} \\ \mathbb{Q}_{\ell} & V=\Lambda_{0}^{3} H_{\mathbb{Q}_{\ell}} \\ 0 & \text { otherwise. }\end{cases}
$$

(iii) If $V$ is a $\operatorname{GSp}(H)$-module satisfying $V^{\mathrm{Sp}(H)}=0$, then there is a natural inclusion

$$
H^{1}\left(G_{k}, H^{1}\left(G_{K}^{\text {geom }}, V\right)\right) \hookrightarrow H^{2}\left(G_{K}, V\right) .
$$

(iv) If $V$ is a $\operatorname{GSp}(H)$-module satisfying $V^{\mathrm{Sp}(H)}=0$, then the restriction mapping $H_{\text {êt }}^{2}\left(\mathcal{M}_{g, n / k}[m], \mathbb{V}\right) \rightarrow H^{2}\left(G_{K}, V\right)$ is injective.

Proof. The extension (19) gives rise to a spectral sequence

$$
E_{2}^{s, t}=H^{s}\left(G_{k}, H^{t}\left(G_{K}^{\text {geom }}, V\right)\right) \Rightarrow H^{s+t}\left(G_{K}, V\right) .
$$

There is therefore an exact sequence

$$
\begin{aligned}
0 \rightarrow H^{1}\left(G_{k}, H^{0}\left(G_{K}^{\text {geom }}, V\right)\right) \rightarrow H^{1}\left(G_{K}, V\right) \\
\\
\rightarrow H^{0}\left(G_{k}, H^{1}\left(G_{K}^{\text {geom }}, V\right)\right) \rightarrow H^{2}\left(G_{k}, H^{0}\left(G_{K}^{\text {geom }}, V\right)\right) .
\end{aligned}
$$

Observe that

$$
H^{\bullet}\left(G_{K}^{\text {geom }}, V\right) \cong \underset{U}{\lim } H_{\text {ét }}^{\bullet}(U, \mathbb{V}),
$$

where $U$ ranges over the Zariski open subsets of $T:=\mathcal{M}_{g, n / \bar{k}}[m]$ that are defined over $k$. 
The Gysin sequence implies that if $U=T-Z$, then there is an exact sequence

$$
0 \rightarrow H_{\mathrm{èt}}^{1}\left(T, \mathbb{Q}_{\ell}\right) \rightarrow H_{\mathrm{et}}^{1}\left(U, \mathbb{Q}_{\ell}\right) \rightarrow \operatorname{Ind}_{G_{k_{S}}}^{G_{k}} \mathbb{Q}_{\ell}(-1)
$$

of $G_{k}$-modules, where $S$ is the set of components of $Z$ that have codimension 1 in $T$ and $G_{k_{S}}$ is the kernel of the action of $G_{k}$ on $S$. Since $H_{\text {ét }}^{1}(T, \mathbb{Q})=0$ for all $g \geq 3$, 14, Prop. 5.2], this implies that $H_{\text {ét }}^{1}\left(U, \mathbb{Q}_{\ell}\right)$ is a $G_{k^{-}}$-submodule of $\operatorname{Ind}_{G_{k_{S}}}^{G_{k}} \mathbb{Q}_{\ell}(-1)$. Therefore $H^{0}\left(G_{k}, H_{\text {ét }}^{1}\left(U, \mathbb{Q}_{\ell}(r)\right)\right)$ vanishes for all $r \neq 1$, and $H^{0}\left(G_{k}, H^{1}\left(G_{K}^{\text {geom }}, \mathbb{Q}_{\ell}(r)\right)\right)$ thus vanishes as well. Assertion (ii) now follows from the exactness of (22).

It suffices to prove (iii) and (iv) when $V$ is a geometrically non-trivial, irreducible $\operatorname{GSp}(H)$-module. For the remainder of the proof, we will assume this to be the case. This assumption implies that $H^{0}\left(G_{K}^{\text {geom }}, V\right)=0$, so that the bottom row of the spectral sequence (21) vanishes. Theorem 16.1 and [14, Proposition 5.2] imply that $\operatorname{Spec} K \rightarrow U \rightarrow T$ induce isomorphisms

$$
\begin{aligned}
& H^{1}\left(G_{K}^{\text {geom }}, H_{\mathbb{Q}_{\ell}}(r)\right) \cong H_{\text {ét }}^{1}\left(U, \mathbb{H}_{\mathbb{Q}_{\ell}}(r)\right) \cong H_{\text {èt }}^{1}\left(T, \mathbb{H}_{\mathbb{Q}_{\ell}}(r)\right) \cong \mathbb{Q}_{\ell}(r)^{n}, \\
& H^{1}\left(G_{K}^{\text {geom }}, \Lambda_{0}^{3} H_{\mathbb{Q}_{\ell}}(r)\right) \cong H_{\text {êt }}^{1}\left(U, \Lambda_{0}^{3} \mathbb{H}_{\mathbb{Q}_{\ell}}(r)\right) \cong H_{\text {èt }}^{1}\left(T, \Lambda_{0}^{3} \mathbb{H}_{\mathbb{Q}_{\ell}}(r)\right) \cong \mathbb{Q}_{\ell}(r)
\end{aligned}
$$

and $H^{1}\left(G_{K}, V\right)=H_{\text {ét }}^{1}(U, \mathbb{V})=H_{\text {èt }}^{1}(T, \mathbb{V})=0$ for all other geometrically non-trivial, irreducible $\operatorname{GSp}\left(H_{\mathbb{Q}_{\ell}}\right)$-modules $V$. Assertion (iii) now follows directly from (22).

As observed above, the fact that $V$ is a geometrically non-trivial, irreducible representation implies that the bottom row of the spectral sequence (21) vanishes. Consequently, there is an exact sequence

$$
\begin{aligned}
0 \rightarrow H^{1}\left(G_{k}, H^{1}\left(G_{K}^{\text {geom }}, V\right)\right) \rightarrow H^{2}\left(G_{K}, V\right) \\
\quad \rightarrow H^{0}\left(G_{k}, H^{2}\left(G_{K}^{\text {geom }}, V\right)\right) \rightarrow H^{2}\left(G_{k}, H^{1}\left(G_{K}^{\text {geom }}, V\right)\right) .
\end{aligned}
$$

Assertion (iii) follows.

To prove (iv), consider the spectral sequence of étale cohomology that converges to $H_{\text {ét }}^{\bullet}(T, \mathbb{V})$. Since $V$ is a geometrically non-trivial, irreducible $\operatorname{GSp}(H)$-module, $H^{0}\left(T \otimes_{k} \bar{k}, \mathbb{V}\right)=0$. The map Spec $K \rightarrow T$ induces a map of spectral sequences, and thus also a map of exact sequences

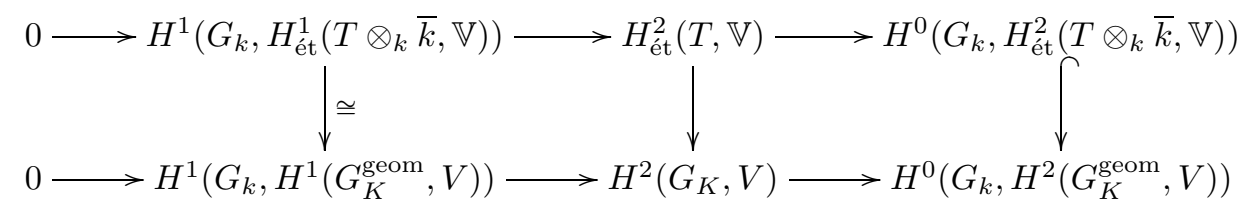

whose second row is part of (23). Theorem 16.1 implies that the left-hand vertical map is an isomorphism and that the right-hand vertical map is an inclusion. Assertion (iv) follows.

Each $\mathrm{Gr}_{-r}^{W} \pi^{\text {un }}$ is a $\operatorname{GSp}(H)$-module. The $G_{K}$ action on it factors through the monodromy representation $G_{K} \rightarrow \operatorname{GSp}\left(H_{\mathbb{Q}_{\ell}}\right)$. Since the image of $G_{K}^{\text {geom }}$ is contained in $\mathrm{Sp}(H)$, the geometric invariant $\left(\mathrm{Gr}_{-r}^{W} \pi^{\mathrm{un}}\right)^{\mathrm{Sp}(H)}$ is a $G_{k}$-module which vanishes when $r$ is odd and is isomorphic to a sum of copies of $\mathbb{Q}_{\ell}(t)$ when $r=2 t$ is even.

Corollary 16.4. Suppose that $g \geq 5$. If $r \geq 2$, then

$$
H^{1}\left(G_{K}, \mathrm{Gr}_{-r}^{W} \pi^{\mathrm{un}}\right) \cong H^{1}\left(G_{k},\left(\mathrm{Gr}_{-r}^{W} \pi^{\mathrm{un}}\right)^{\mathrm{Sp}(H)}\right) .
$$


In particular, when $r>1$ is odd, then $H^{1}\left(G_{K}, \mathrm{Gr}_{-r}^{W} \pi^{\mathrm{un}}\right)$ vanishes. If $r \geq 2$, then the cup product

$$
H^{1}\left(G_{K}, H\right) \otimes H^{1}\left(G_{K}, \mathrm{Gr}_{-2 r}^{W} \pi^{\mathrm{un}}\right) \rightarrow H^{2}\left(G_{K}, \mathrm{Gr}_{-2 r-1}^{W} \pi^{\mathrm{un}}\right),
$$

induced by the commutator $H \otimes \mathrm{Gr}_{-2 r}^{W} \pi^{\mathrm{un}} \rightarrow \mathrm{Gr}_{-2 r-1}^{W} \pi^{\mathrm{un}}$, is injective.

Proof. Suppose that $r \geq 2$. Write

$$
\mathrm{Gr}_{-r}^{W} \pi^{\mathrm{un}}=\left(\mathrm{Gr}_{-r}^{W} \pi^{\mathrm{un}}\right)^{\mathrm{Sp}(H)} \oplus V
$$

where $V$ is a $\operatorname{GSp}(H)$-module. Since $V$ has weight $<-1$ and since each of its irreducible components is geometrically non-trivial, Proposition 16.3 implies that $H^{1}\left(G_{K}, V\right)=0$. It follows that

$$
H^{1}\left(G_{K}, \mathrm{Gr}_{-r}^{W} \pi^{\mathrm{un}}\right) \cong H^{1}\left(G_{K},\left(\mathrm{Gr}_{-r}^{W} \pi^{\mathrm{un}}\right)^{\mathrm{Sp}(H)}\right)
$$

Since $\left(\mathrm{Gr}_{-r}^{W} \pi^{\mathrm{un}}\right)^{\mathrm{Sp}(H)}$ vanishes when $r$ is odd and when $r=2$, and is a direct sum of copies of $\mathbb{Q}_{\ell}(t)$ when $r=2 t$, Proposition 16.3 implies that, when $r \geq 2$,

$$
H^{1}\left(G_{K},\left(\mathrm{Gr}_{-r}^{W} \pi^{\mathrm{un}}\right)^{\mathrm{Sp}(H)}\right) \cong H^{1}\left(G_{k},\left(\mathrm{Gr}_{-r}^{W} \pi^{\mathrm{un}}\right)^{\mathrm{Sp}(H)}\right) .
$$

This proves the first assertion.

Schur's Lemma implies that all $\mathrm{Sp}(H)$-submodules of $H \otimes\left(\mathrm{Gr}_{-r}^{W} \pi^{\mathrm{un}}\right)^{\mathrm{Sp}(H)}$ are of the form $H \otimes B$, where $B$ is a subspace of $\left(\operatorname{Gr}_{-r}^{W} \pi^{\text {un }}\right)^{\operatorname{Sp}(H)}$. Since the Lie algebra $\mathrm{Gr}_{\bullet}^{W} \pi^{\mathrm{un}}$ is generated by $H=\mathrm{Gr}_{-1}^{W} \pi^{\mathrm{un}}$ and has trivial center [34, p. 201], the commutator map $H \otimes\left(\mathrm{Gr}_{-r}^{W} \pi^{\mathrm{un}}\right)^{\mathrm{Sp}(H)} \rightarrow \mathrm{Gr}_{-r-1}^{W} \pi^{\mathrm{un}}$ is injective. Since $\mathrm{Gr}_{-r-1}^{W} \pi^{\mathrm{un}}$ is a semi-simple $G_{K}$-module,

$$
H^{2}\left(G_{K}, H \otimes\left(\mathrm{Gr}_{-r-1}^{W} \pi^{\mathrm{un}}\right)^{\mathrm{Sp}(H)}\right) \rightarrow H^{2}\left(G_{K}, \mathrm{Gr}_{-r-1}^{W} \pi^{\mathrm{un}}\right)
$$

is injective. To prove the last assertion, it thus suffices to show that the cup product

$$
H^{1}\left(G_{K}, H\right) \otimes H^{1}\left(G_{K},\left(\mathrm{Gr}_{-r}^{W} \pi^{\mathrm{un}}\right)^{\mathrm{Sp}(H)}\right) \rightarrow H^{2}\left(G_{K}, H \otimes\left(\mathrm{Gr}_{-r}^{W} \pi^{\mathrm{un}}\right)^{\mathrm{Sp}(H)}\right)
$$

is injective.

Proposition 16.3 (iii) implies that $H^{1}\left(G_{K}, H\right)=H^{1}\left(G_{K}^{\text {geom }}, H\right)$. This is a trivial $G_{k}$-module. The cup product (24) is thus the composite of the mappings:

$$
\begin{aligned}
& H^{1}\left(G_{K}, H\right) \otimes H^{1}\left(G_{k},\left(\mathrm{Gr}_{-r}^{W} \pi^{\mathrm{un}}\right)^{\mathrm{Sp}(H)}\right) \\
& \stackrel{\simeq}{\rightarrow} H^{1}\left(G_{K}^{\text {geom }}, H\right) \otimes H^{1}\left(G_{k},\left(\mathrm{Gr}_{-r}^{W} \pi^{\mathrm{un}}\right)^{\mathrm{Sp}(H)}\right) \\
& \cong H^{1}\left(G_{k}, H^{1}\left(G_{K}^{\text {geom }}, H\right) \otimes\left(\mathrm{Gr}_{-r}^{W} \pi^{\mathrm{un}}\right)^{\mathrm{Sp}(H)}\right) \\
& \cong H^{1}\left(G_{k}, H^{1}\left(G_{K}^{\text {geom }}, H \otimes\left(\mathrm{Gr}_{-r}^{W} \pi^{\mathrm{un}}\right)^{\mathrm{Sp}(H)}\right)\right) \\
& \subseteq H^{2}\left(G_{K}, H \otimes\left(\mathrm{Gr}_{-r}^{W} \pi^{\mathrm{un}}\right)^{\mathrm{Sp}(H)}\right),
\end{aligned}
$$

which is injective as Proposition 16.3(iv) implies that the last mapping is injective.

\section{Computation of $H^{\bullet}\left(\mathfrak{g}_{K}, \mathrm{Gr}_{\bullet}^{W} \mathfrak{p}\right)$}

The computation of the cohomology groups $H^{\bullet}\left(\mathfrak{g}_{K}, \mathrm{Gr}_{-r}^{W} \mathfrak{p}\right)$ in low degrees is needed for the application of the non-abelian cohomology of Section 14 to the proofs of Theorems 2 and 3 . Throughout this section, we assume that $\chi_{\ell}: G_{k} \rightarrow \mathbb{Z}_{\ell}^{\times}$has infinite image.

Since $\operatorname{GSp}(H)$ is connected, Proposition 6.8 and Remark 6.9 imply that

$$
\left.H^{j}\left(\mathfrak{g}_{g, n}, V\right) \cong \operatorname{Hom}_{\mathrm{GSp}(H)}\left(H_{j}\left(\mathfrak{u}_{g, n}\right), V\right)\right)
$$


for all finite dimensional $\operatorname{GSp}(H)$-modules $V$. In particular, the projections

$$
\mathbf{c}, \mathbf{d}_{j}, \mathbf{e}_{j}, \mathbf{e}_{i j} \in \operatorname{Hom}_{\mathrm{GSp}(H)}\left(H_{2}\left(\mathfrak{u}_{g, n}\right), \Lambda_{0}^{2} H\right)
$$

defined in display (12), Section 9, can be regarded as elements of $H^{2}\left(\mathfrak{g}_{g, n}, \Lambda_{0}^{2} H\right)$. We will also regard them as elements of $H^{2}\left(\mathfrak{g}_{K}, \Lambda_{0}^{2} H\right)$ via the natural homomorphism

$$
H^{2}\left(\mathfrak{g}_{g, n}, \Lambda_{0}^{2} H\right) \rightarrow H^{2}\left(\mathfrak{g}_{K}, \Lambda_{0}^{2} H\right) .
$$

Proposition 17.1. If $g \geq 5$ and $n \geq 0$, then

$$
H^{1}\left(\mathfrak{g}_{K}, \mathrm{Gr}_{-r}^{W} \mathfrak{p}\right) \cong \begin{cases}\mathbb{Q}_{\ell}{ }^{n} & r=1 \\ 0 & 2<r<6 \text { and } r \text { odd }>6 \\ H^{1}\left(G_{k},\left(\mathrm{Gr}_{-r}^{W} \pi^{\mathrm{un}}\right)^{\mathrm{Sp}(H)}\right) & r>2 \text { even }\end{cases}
$$

When $r=2, H^{2}\left(\mathfrak{g}_{K}, \mathrm{Gr}_{-2}^{W} \pi^{\text {un }}\right)$ is isomorphic to $H^{2}\left(\mathfrak{g}_{g, n}, \Lambda_{0}^{2} H\right)$ and is generated by the classes $\mathbf{c}, \mathbf{d}_{j}, \mathbf{e}_{j}(1 \leq j \leq n), \mathbf{e}_{i j}(1 \leq i \leq j \leq n)$, which are subject to the relations

$$
\mathbf{c}+\mathbf{d}_{j}+\mathbf{e}_{j}=0, \quad j=1, \ldots, n .
$$

Consequently $\left\{\mathbf{c}, \mathbf{e}_{i}, \mathbf{e}_{i j}: 1 \leq i \leq j \leq n\right\}$ is a basis of $H^{2}\left(\mathfrak{g}_{K}, \Lambda_{0}^{2} H\right)$.

Proof. The exponential map induces a $\mathcal{G}_{K}$-module isomorphism $\mathrm{Gr}_{-r}^{W} \mathfrak{p} \rightarrow \mathrm{Gr}_{-r}^{W} \pi^{\mathrm{un}}$. We will denote this coefficient module by $\mathrm{Gr}_{-r}^{W} \pi^{\text {un }}$ in group cohomology, and by $\mathrm{Gr}_{-r}^{W} \mathfrak{p}$ in Lie algebra cohomology. Proposition 16.3 implies that the natural map

$$
H^{j}\left(\mathfrak{g}_{K}, \mathrm{Gr}_{-r}^{W} \mathfrak{p}\right) \rightarrow H^{j}\left(G_{K}, \mathrm{Gr}_{-r}^{W} \pi^{\mathrm{un}}\right)
$$

is an isomorphism when $j=1$ and injective when $j=2$. The computation of $H^{1}\left(\mathfrak{g}_{K}, \mathrm{Gr}_{-r}^{W} \mathfrak{p}\right)$ follows directly from Proposition 16.3. Corollary [9.3, and Corollary 16.4 .

Proposition 6.8 implies that $\operatorname{Spec} K \rightarrow \mathcal{M}_{g, n / k}$ induces an inclusion

$$
H_{\text {ét }}^{2}\left(\mathcal{M}_{g, n / k}, \Lambda_{0}^{2} H\right) \hookrightarrow H^{2}\left(G_{K}, \Lambda_{0}^{2} H\right)
$$

and therefore an injection

$$
H^{2}\left(\mathfrak{g}_{g, n}, \Lambda_{0}^{2} H\right) \rightarrow H^{2}\left(\mathfrak{g}_{K}, \Lambda_{0}^{2} H\right) .
$$

Apply $\operatorname{Hom}_{\mathrm{GSp}(H)}\left(, \Lambda_{0}^{2} H\right)$ to the exact sequence in Lemma 6.7 with $\mathfrak{u}=\mathfrak{u}_{K}$ and $\mathfrak{u}_{g, n}$ to obtain the commutative diagram

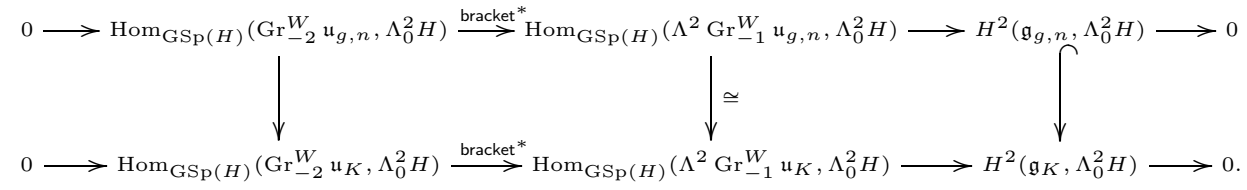

The rows are exact because $H^{1}\left(\mathfrak{g}_{K}, \Lambda_{0}^{2} H\right)$ and $H^{1}\left(\mathfrak{g}_{g, n}, \Lambda_{0}^{2} H\right)$ both vanish when $g \geq 5$. This implies that (25) is an isomorphism, which implies that the left-hand vertical map is an isomorphism. The result now follows from Proposition 9.12, which implies that $\left\{\mathbf{c}, \mathbf{d}_{i}, \mathbf{e}_{i}, \mathbf{e}_{i j}: 1 \leq i \leq j \leq n\right\}$ is a basis of the middle group, and Proposition 9.13, which implies that the relations are $\left\{\mathbf{c}+\mathbf{d}_{i}+\mathbf{e}_{i}=0: 1 \leq i \leq n\right\}$. 
Since $H^{1}\left(G_{K}, \operatorname{Gr}_{-r}^{W} \pi^{\text {un }}\right)$ is finite dimensional if and only if $H^{1}\left(G_{k}, \mathbb{Q}_{\ell}(r / 2)\right)$ is finite dimensional, the previous proposition and Corollary 16.4 imply:

Proposition 17.2. If $1 \leq r<6$ or if $r$ is odd, then for all fields $k$ of characteristic zero, $H^{1}\left(\mathfrak{g}_{K}, \mathrm{Gr}_{-r}^{W} \mathfrak{p}\right)$ is finite dimensional. If $r=2 s \geq 6$ is even and if $H^{1}\left(G_{k}, \mathbb{Q}_{\ell}(s)\right)$ is finite dimensional, then $H^{1}\left(\mathfrak{g}_{K}, \mathrm{Gr}_{-r}^{W} \mathfrak{p}\right)$ is finite dimensional.

This allows us to apply the non-abelian cohomology of Section 14 when $k$ is a number field or a finite extension of $\mathbb{Q}_{p}$ provided $p \neq \ell$. The following result is needed for the computation of the connecting morphism in non-abelian cohomology.

Proposition 17.3. If $g \geq 5$ and $n \geq 1$, then the map

$$
H^{1}\left(\mathfrak{g}_{K}, \operatorname{Gr}_{-1}^{W} \mathfrak{p}\right) \otimes H^{1}\left(\mathfrak{g}_{K}, \operatorname{Gr}_{-r}^{W} \mathfrak{p}\right) \rightarrow H^{2}\left(\mathfrak{g}_{K}, \operatorname{Gr}_{-r-1}^{W} \mathfrak{p}\right),
$$

induced by the bracket $\mathrm{Gr}_{-1}^{W} \mathfrak{p} \otimes \mathrm{Gr}_{-r}^{W} \mathfrak{p} \rightarrow \mathrm{Gr}_{-r-1}^{W} \mathfrak{p}$, is injective.

Proof. This follows from Corollary 16.4 and the fact that the diagram

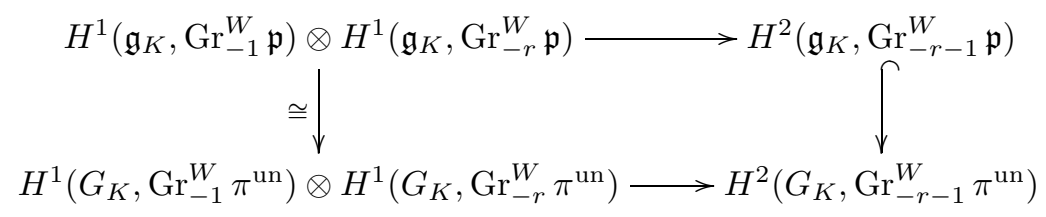

commutes, and the fact that the left-hand vertical map is an isomorphism and the right-hand vertical map is an inclusion.

\section{Proofs of Theorems 2 and 3}

We are now ready to combine the non-abelian cohomology of Section 14 with the subsequent computations to prove Theorems 2 and 3 . Throughout we assume that $g \geq 5$ and that $r \geq 1$ and that the image of $\chi_{\ell}: G_{k} \rightarrow \mathbb{Z}_{\ell}^{\times}$is infinite. We will assume that $H^{1}\left(\mathfrak{g}_{K}, \mathrm{Gr}_{-s}^{W} \mathfrak{p}\right)$ is finite dimensional whenever $1 \leq s \leq r$ to ensure that the scheme $H_{\text {nab }}^{1}\left(G_{K}, \pi^{\mathrm{un}} / W_{-r-1}\right)$ is defined. Conditions under which this is the case are given in Proposition 17.2. They are satisfied, for example, when $k$ is a number field or a finite extensions of $\mathbb{Q}_{p}$ where $p \neq \ell$.

When $n \geq 1$, denote by $s_{j}$ the section of $\mathcal{G}_{C} \rightarrow \mathcal{G}_{K}$ induced by the $j$ th tautological point $x_{j}$ of $C$. There are thus maps

$$
\iota_{r}:\left\{s_{1}, \ldots, s_{n}\right\} \rightarrow H_{\text {nab }}^{1}\left(\mathcal{G}_{K}, \pi^{\mathrm{un}} / W_{-r-1}\right)\left(\mathbb{Q}_{\ell}\right)
$$

for each $r>0$. When $n=0$, we consider $\left\{s_{1}, \ldots, s_{n}\right\}$ to be the empty set. The maps $\iota_{r}$ are compatible in the sense that

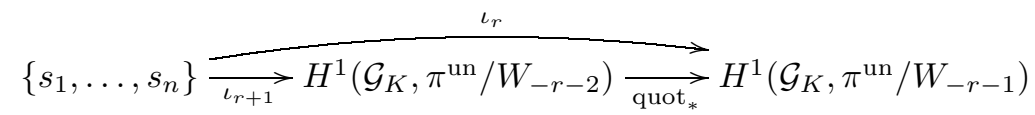

commutes.

When $r=1, H^{1}\left(\mathcal{G}_{K}, \pi^{\text {un }} / W_{-2}\right)$ is a principal homogeneous space over

$$
H^{1}\left(\mathfrak{g}_{K}, \operatorname{Gr}_{-1}^{W} \mathfrak{p}\right)=H^{1}\left(\mathfrak{g}_{K}, H\right)=\mathbb{Q}_{\ell} \kappa_{1} \oplus \cdots \oplus \mathbb{Q}_{\ell} \kappa_{n} .
$$

The first task it to fix a natural identification

$$
H^{1}\left(\mathfrak{g}_{K}, \mathrm{Gr}_{-1}^{W} \mathfrak{p}\right) \stackrel{\widetilde{\simeq}}{\rightarrow} H_{\text {nab }}^{1}\left(\mathcal{G}_{K}, \pi^{\mathrm{un}} / W_{-2}\right) .
$$


Proposition 14.5 implies that elements of $H_{\text {nab }}^{1}\left(\mathcal{G}_{K}, \pi^{\text {un }} / W_{-2}\right)$ correspond to $\operatorname{GSp}(H)$-invariant sections of $\mathrm{Gr}_{-1}^{W} \mathfrak{u}_{C} \rightarrow \mathrm{Gr}_{-1}^{W} \mathfrak{u}_{K}$. Theorem 9.11 implies that these correspond to the $\operatorname{GSp}(H)$-invariant sections of the map $\epsilon_{n}: \Lambda_{n+1}^{3} H \rightarrow \Lambda_{n}^{3} H$ that takes $\left(v ; u_{0}, u_{1}, \ldots, u_{n}\right)$ to $\left(v ; u_{1}, \ldots, u_{n}\right)$. We identify $H_{\text {nab }}^{1}\left(\mathcal{G}_{K}, \pi^{\text {un }} / W_{-2}\right)$ with $\mathbb{A}_{\mathbb{Q}_{\ell}}^{n}$ by identifying the $\operatorname{GSp}(H)$-invariant section

$$
\left(v ; u_{1}, \ldots, u_{n}\right) \mapsto\left(v: \sum_{j=1}^{n} a_{j} u_{j}, u_{1}, \ldots, u_{n}\right)
$$

of $\epsilon_{n}$ with $\left(a_{1}, \ldots, a_{n}\right)$. Corollary 12.6 now implies:

Lemma 18.1. With this identification,

$$
(2 g-2) \iota_{1}\left(s_{j}\right)=\kappa_{j} \in H_{\text {nab }}^{1}\left(\mathcal{G}_{K}, \pi^{\text {un }} / W_{-2}\right)\left(\mathbb{Q}_{\ell}\right),
$$

where $1 \leq j \leq n$. Consequently, $\iota_{r}$ is injective for each $r \geq 1$.

The computation of $\delta: H_{\text {nab }}^{1}\left(\mathcal{G}_{K}, \pi^{\mathrm{un}} / W_{-2}\right) \rightarrow H^{2}\left(\mathfrak{g}_{K}, \mathrm{Gr}_{-2}^{W} \mathfrak{p}\right)$ is a reformulation of Lemma 10.5

Lemma 18.2. If $g \geq 5$, then the connecting morphism

$$
\delta: H_{\text {nab }}^{1}\left(\mathcal{G}_{K}, \pi^{\mathrm{un}} / W_{-2}\right) \rightarrow H^{2}\left(\mathfrak{g}_{K}, \mathrm{Gr}_{-2}^{W} \mathfrak{p}\right)
$$

is given by

$$
\delta\left(\sum_{j=1}^{n} a_{j} \kappa_{j}\right) /(2 g-2)=\sum_{i<j} a_{i} a_{j} \mathbf{e}_{i j}+\sum_{i}\left(a_{i}^{2}-a_{i}\right) \mathbf{e}_{i}+\left(1-\sum_{i} a_{i}\right) \mathbf{c} .
$$

Proof. This follows directly from the construction of $\delta$ given in Section 14.2, the cohomology computation in Proposition 17.1, and the formula for the bracket given in Lemma 10.5.

Corollary 18.3. If $g \geq 5$, then

$$
\iota_{2}:\left\{s_{1}, \ldots, s_{n}\right\} \rightarrow H_{\text {nab }}^{1}\left(\mathcal{G}_{K}, \pi^{\text {un }} / W_{-3}\right)\left(\mathbb{Q}_{\ell}\right)
$$

is a bijection.

Proof. Theorem 14.3 implies that $H^{1}\left(\mathcal{G}_{K}, \pi^{\text {un }} / W_{-3}\right)$ is the inverse image of 0 under

$$
\delta: H^{1}\left(\mathcal{G}_{K}, H\right) \rightarrow H^{2}\left(\mathfrak{g}_{K}, \mathrm{Gr}_{-2}^{W} \mathfrak{p}\right) .
$$

The previous result and the Chinese Remainder Theorem imply that

$$
\begin{aligned}
\delta^{-1}(0) & =\left\{(2 g-2)^{-1} \sum_{j=0}^{n} a_{j} \kappa_{j}: a_{i} a_{j}=\delta_{i, j} a_{j}, \sum_{i} a_{i}=1\right\} \\
& =\left\{\kappa_{1} /(2 g-2), \ldots, \kappa_{n} /(2 g-2)\right\} \times \operatorname{Spec} \mathbb{Q}_{\ell} .
\end{aligned}
$$

The result follows as $\iota_{2}\left(s_{j}\right)=\kappa_{j} /(2 g-2)$.

Proof of Theorem 2. By Proposition [7.3, a section of $\pi_{1}(C, \bar{x}) \rightarrow G_{K}$ induces a section of $\mathcal{G}_{C} / W_{-3} \pi^{\text {un }} \rightarrow \mathcal{G}_{K}$. But the previous result implies that there are no such sections when $n=0$.

Another consequence is the following version of the Section Conjecture. The theorem shows that Kim's program [29] works well in the case where the curve is the generic curve of type $(g, n)$ with a level $m \geq 1$ structure and $g \geq 5$. 
Theorem 18.4. Suppose that $k$ is a field of characteristic zero and that $\ell$ is a prime number for which the image of the $\ell$-adic cyclotomic character $\chi_{\ell}$ is infinite. Let $K=k\left(\mathcal{M}_{g, n}[m]\right)$ and $C$ be the restriction of the universal curve over $\mathcal{M}_{g, n}[m]$ to its generic point. If $g \geq 5$ and $n \geq 0$, then $C(K)=\left\{x_{1}, \ldots, x_{n}\right\}$ and the function

$$
\operatorname{sect}_{r}^{\mathrm{un}}: C(K) \rightarrow H_{\mathrm{nab}}^{1}\left(G_{K}, \pi^{\mathrm{un}} / W_{-r-1}\right)\left(\mathbb{Q}_{\ell}\right)
$$

that takes a rational point $x$ to the conjugacy class of the section $s_{r, x}^{\mathrm{un}}$ of

$$
\widetilde{G}_{C}^{\mathbb{Q}_{\ell}} / W_{-r-1} \pi^{\mathrm{un}}\left(\mathbb{Q}_{\ell}\right) \rightarrow G_{K}
$$

induced by $s_{x}$ is a bijection when $2 \leq r \leq 5$. If $H^{1}\left(G_{k}, \mathbb{Q}_{\ell}(t)\right)$ vanishes for all $t \geq 3$, such as when $k$ is a finite extension of $\mathbb{Q}_{p}, p \neq \ell$ [35. VII, §3], then sect $_{r}^{\text {un }}$ is a bijection for all $r \in[3, \infty]$.

Proof. We know from Theorem 1 that $C(K)=\left\{x_{1}, \ldots, x_{n}\right\}$. From Proposition 15.2 we know that $H_{\text {nab }}^{1}\left(\mathcal{G}_{K}, \pi^{\text {un }} / W_{-r-1}\right) \rightarrow H_{\text {nab }}^{1}\left(G_{K}, \pi^{\text {un }} / W_{-r-1}\right)$ is an isomorphism. The result then follows from the exact sequence of Theorem 14.3 and the computations of Proposition 17.1

Additional work is required when $H^{1}\left(G_{k}, \mathbb{Q}_{\ell}(t)\right)$ does not vanish for all $t \geq 3$. This is the case when $k$ is a number field, for example.

Proposition 18.5. Suppose that $r \geq 2$ and that $H^{1}\left(G_{k}, \mathbb{Q}_{\ell}(t)\right)$ is finite dimensional whenever $3 \leq 2 t \leq r$. If $g \geq 5$, then there are natural isomorphisms

$$
\begin{aligned}
& H_{\text {nab }}^{1}\left(G_{K}, \pi^{\mathrm{un}} / W_{-r-1}\right) \cong H_{\text {nab }}^{1}\left(\mathcal{G}_{K}, \pi^{\mathrm{un}} / W_{-r-1}\right) \\
& \cong\left\{s_{1}, \ldots, s_{n}\right\} \times H^{1}\left(\mathfrak{g}_{K}, \mathrm{Gr}_{-r}^{W} \mathfrak{p}\right) \\
& \cong \begin{cases}\left\{s_{1}, \ldots, s_{n}\right\} \times \operatorname{Spec} \mathbb{Q}_{\ell} & r \text { odd }, \\
\left\{s_{1}, \ldots, s_{n}\right\} \times H^{1}\left(G_{k},\left(\operatorname{Gr}_{-r}^{W} \pi^{\mathrm{un}}\right)^{\mathrm{Sp}(H)}\right) & r \text { even, }\end{cases}
\end{aligned}
$$

where $\iota_{m}\left(s_{j}\right)$ corresponds to $s_{j}$ when $r$ is odd and to $\left(s_{j}, 0\right)$ when $r$ is even. When $r=2 t$, there is a commutative diagram

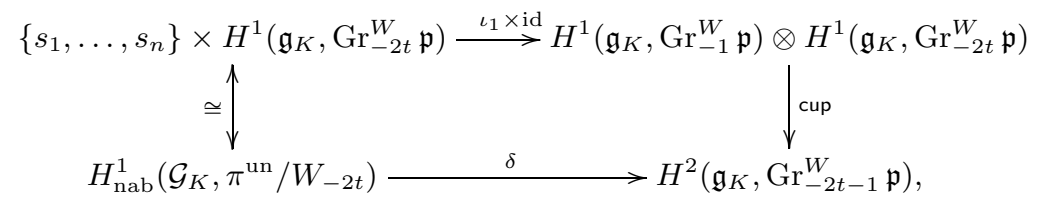

where cup is induced by the bracket $\mathrm{Gr}_{-1}^{W} \mathfrak{p} \otimes \mathrm{Gr}_{-2 t}^{W} \mathfrak{p} \rightarrow \mathrm{Gr}_{-2 t-1}^{W} \mathfrak{p}$.

Proof. We prove this result by induction on $r$ using the exact sequence of Theorem 14.3. The case $r=2$ is established in Corollary 18.3 Observe that the composition

$$
\left\{s_{1}, \ldots, s_{n}\right\} \stackrel{\iota_{r}}{\longrightarrow} H^{1}\left(\mathcal{G}_{K}, \pi^{\mathrm{un}} / W_{-r-1}\right)\left(\mathbb{Q}_{\ell}\right) \stackrel{\delta}{\longrightarrow} H^{2}\left(\mathfrak{g}_{K}, \mathrm{Gr}_{-r-1}^{W} \mathfrak{p}\right)
$$

of $\iota_{r}$ with the connecting morphism $\delta$ is trivial for all $r>1$. Since $H^{1}\left(\mathfrak{g}, \operatorname{Gr}_{-r}^{W} \mathfrak{p}\right)=0$ for $2 \leq r<6$, the result also holds when $r<6$.

Next observe that if the result is true for $r=2 t-1>2$, it is also true for $r=2 t$ as the exact sequence of non-abelian cohomology implies that $H_{\text {nab }}^{1}\left(\mathcal{G}, \pi / W_{-2 t-1}\right)$ is a principal $H^{1}\left(\mathfrak{g}_{K}, \mathrm{Gr}_{-2 t}^{W} \mathfrak{p}\right)$ bundle over $H_{\text {nab }}^{1}\left(\mathcal{G}_{K}, \pi^{\mathrm{un}} / W_{-2 t-2} \pi^{\mathrm{un}}\right)$, which, by induction, is $\left\{s_{1}, \ldots, s_{n}\right\}$. 
To prove that if the result is true for $r=2 t$, then it is true for $r=2 t+1$, it suffices to prove the last assertion as Proposition 17.3 then implies that $\delta$ is an imbedding on each $s_{j} \times H^{1}\left(\mathfrak{g}_{K}, \mathrm{Gr}_{-2 t}^{W} \mathfrak{p}\right)$ that takes $\left(s_{j}, 0\right)$ to 0 . But this follows from the description of $\delta$ given in Section 14.2 .

Combining Theorem 1 with the fact that $H_{\text {nab }}^{1}\left(G_{K}, \pi^{\text {un }}\right)$ is the projective limit of the $H_{\text {nab }}^{1}\left(G_{K}, \pi^{\text {un }} / W_{-r}\right)$, we obtain:

Theorem 18.6. Suppose that $k$ is a field of characteristic zero and that $\ell$ is a prime number for which the image of the $\ell$-adic cyclotomic character $\chi_{\ell}$ is infinite. Let $K=k\left(\mathcal{M}_{g, n}[m]\right)$ and $C$ be the restriction of the universal curve over $\mathcal{M}_{g, n / k}[m]$ to its generic point. If $g \geq 5$ and $n \geq 0$ and if $H^{1}\left(G_{k}, \mathbb{Q}_{\ell}(s)\right)$ is finite dimensional for all $s \geq 3$, then $C(K)=\left\{x_{1}, \ldots, x_{n}\right\}$ and the function

$$
\operatorname{sect}_{\infty}^{\text {un }}: C(K) \rightarrow H_{\text {nab }}^{1}\left(G_{K}, \pi^{\text {un }}\right)\left(\mathbb{Q}_{\ell}\right)
$$

that takes a rational point to the conjugacy class of the section of

$$
\widetilde{G}_{C}^{\mathbb{Q}_{\ell}} / W_{-r-1} \pi^{\mathrm{un}}\left(\mathbb{Q}_{\ell}\right) \rightarrow G_{K}
$$

induced by $s_{x}$ is a bijection.

This completes the proof of Theorem 3. With minor modifications, one can prove the following generalization.

Let $T$ be a section of dimension $\geq 3$ of $\mathcal{M}_{g, n / k}[m]$ by a generic complete intersection as described in Remark 16.2. Suppose that $T$ is defined over $k$ and geometrically connected. Denote the restriction of the universal curve over $\mathcal{M}_{g, n / k}[m]$ to $T$ by $C \rightarrow T$. Set $K=k(T)$.

Theorem 18.7. Suppose that $\ell$ is a prime number and that the image of the $\ell$-adic cyclotomic character is infinite. If $g \geq 5, n \geq 0$ and $m \geq 1$ and if $H^{1}\left(G_{k}, \mathbb{Q}_{\ell}(r)\right)$ is finite dimensional for all $r>1$, then

$$
C(K) \rightarrow H_{\text {nab }}^{1}\left(G_{K}, \pi^{\mathrm{un}}\right)\left(\mathbb{Q}_{\ell}\right)
$$

is an isomorphism.

The proof is left to the interested reader. The main point is that the computations of Section 16 hold in this more general situation. The rest of the argument is identical.

\section{Appendix. A Hodge theoretic lemma}

Suppose that $T$ is a complex algebraic manifold and that $\mathbb{H}$ is a polarized variation of Hodge structure (PVHS) of negative weight. Suppose that the $\mathbb{Z}$-local system underlying $\mathbb{H}$ is torsion free. Here we will construct the sequence

$$
0 \rightarrow \operatorname{Ext}_{\mathcal{H}}^{1}\left(\mathbb{Z}(0), H^{0}\left(T, \mathbb{H}_{\mathbb{Z}}\right)\right) \stackrel{j}{\rightarrow} \operatorname{Ext}_{\mathcal{H}(T)}^{1}\left(\mathbb{Z}(0)_{T}, \mathbb{H}_{\mathbb{Z}}\right) \stackrel{\delta}{\rightarrow} H^{1}\left(T, \mathbb{H}_{\mathbb{Z}}\right)
$$

used in the proof of Proposition 11.2 and prove that it is exact. The proof below is short and direct. A stronger result can be deduced from general results proved in 24 in which the image of $\delta$ is shown the be the appropriate set of Hodge classes.

The map $\delta$ takes a VMHS $\mathbb{V}$ that is an extension

$$
0 \rightarrow \mathbb{H} \rightarrow \mathbb{V} \rightarrow \mathbb{Z}(0)_{T} \rightarrow 0
$$


to the corresponding extension of local systems, which is an element of

$$
\operatorname{Ext}_{\mathcal{L}(T)}^{1}(\mathbb{Z}, \mathbb{H}) \cong H^{1}(T, \mathbb{H})
$$

where $\mathcal{L}(T)$ denotes the category of $\mathbb{Z}$-local systems over $T$.

The map $j$ is defined by pushout. By the theorem of the fixed part for PVHS, $H^{0}(T, \mathbb{H})\left[39\right.$ has a pure Hodge structure and the inclusion $H^{0}(T, \mathbb{H}) \hookrightarrow H_{t}$ is a morphism of HS for all $t \in T$. Thus $H^{0}(T, \mathbb{H})$ may be regarded as a constant sub PVHS of $\mathbb{H}$ over $T$. An extension

$$
0 \rightarrow H^{0}(T, \mathbb{H}) \rightarrow V \rightarrow \mathbb{Z}(0) \rightarrow 0
$$

in the category of MHS can be viewed as a constant VMHS over $T$. One can push it out along the inclusion $H^{0}(T, \mathbb{H}) \hookrightarrow \mathbb{H}$ to obtain an extension of $\mathbb{Z}(0)_{T}$ by $\mathbb{H}$ :

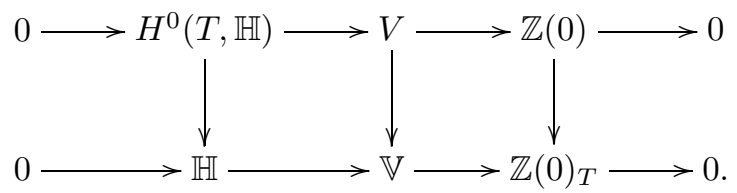

The map $j$ takes the extension (27) to this extension. Injectivity will follow from the computation below.

We say that an extension

$$
0 \rightarrow \mathbb{H} \rightarrow \mathbb{V} \rightarrow \mathbb{Z}(0)_{T} \rightarrow 0
$$

in the category $\mathcal{H}(T)$ of admissible VMHS over $T$ is topologically trivial if it splits in the category $\mathcal{L}(T)$ of local systems over $T$. The kernel of $\delta$ consists of those extensions $\mathbb{V}$ that are topologically trivial.

To prove exactness, it suffices to show that every topologically trivial extension of $\mathbb{Z}(0)_{T}$ by $\mathbb{H}$ arises from an element of $\operatorname{Ext}_{\mathcal{H}}^{1}\left(\mathbb{Z}(0), H^{0}\left(T, \mathbb{H}_{\mathbb{Z}}\right)\right)$ by the pushout construction above. Suppose that $\mathbb{V}$ is an extension of $\mathbb{Z}(0)_{T}$ by $\mathbb{H}$ in $\mathcal{H}(T)$. Then the Theorem of the Fixed Part for VMHS [4] implies that $H^{0}(T, \mathbb{V})$ has a natural MHS. The exact sequence

$$
0 \rightarrow \mathbb{H} \rightarrow \mathbb{V} \rightarrow \mathbb{Z}(0)_{T} \rightarrow 0
$$

gives a long exact sequence

$$
0 \rightarrow H^{0}(T, \mathbb{H}) \rightarrow H^{0}(T, \mathbb{V}) \rightarrow \mathbb{Z}(0) \stackrel{d}{\rightarrow} H^{1}(T, \mathbb{H})
$$

Then $\mathbb{V}$ is a topologically trivial extension of $\mathbb{Z}$ by $\mathbb{H}$ if and only if $d=0$. Thus, when $\mathbb{V}$ is a topologically trivial extension, we have the short exact sequence

$$
0 \rightarrow H^{0}(T, \mathbb{H}) \rightarrow H^{0}(T, \mathbb{V}) \rightarrow \mathbb{Z}(0) \rightarrow 0,
$$

which we regard as an element of $\operatorname{Ext}_{\mathcal{H}}^{1}\left(\mathbb{Z}(0), H^{0}\left(T, \mathbb{H}_{\mathbb{Z}}\right)\right)$. It may be regarded as a subvariation of $\mathbb{V}$ over $T$. This construction defines a function

$$
r: \operatorname{ker} \delta \rightarrow \operatorname{Ext}_{\mathcal{H}}^{1}\left(\mathbb{Z}(0), H^{0}\left(T, \mathbb{H}_{\mathbb{Z}}\right)\right),
$$

which is easily seen to be a homomorphism. It is also easy to check that $r \circ j$ is the identity. To prove exactness of the sequence (26), it suffices to show that $r$ is injective. But this is clear, for if the extension (29) is split, then one has a Hodge splitting $s: \mathbb{Z}(0)_{T} \rightarrow H^{0}(T, \mathbb{V})$ of it. But, regarding $H^{0}(T, \mathbb{V})$ as a constant subvariation of $\mathbb{V}$, we see that $s$ gives a splitting of (28) in $\mathcal{H}(T)$. This implies that ker $r=0$. 


\section{ACKNOWLEDGMENTS}

Much of this work was done in 2009 during visits to MSRI, the Université de Nice and the Newton Institute. I am very grateful to these institutions for their hospitality and support and to Duke University for the sabbatical leave during which this work was completed. I am also grateful to the many mathematicians who took an interest in this work and with whom I had many helpful discussions, particularly Jordan Ellenberg, Minhyong Kim and Makoto Matsumoto. I am also grateful to Arnaud Beauville and Naria Kawazumi, both of whom pointed out the existence and relevance of the works of Hubbard [25] and Earle and Kra [6]. Finally, I owe a huge debt of gratitude to the referee for his/her very thorough reading of the manuscript and his/her numerous detailed comments and corrections.

\section{REFERENCES}

[1] M. Anderson: Exactness properties of profinite completion functors, Topology 13 (1974), 229-239. MR0354882 (50:7359)

[2] J. Birman: Braids, links, and mapping class groups. Annals of Mathematics Studies, No. 82. Princeton University Press, 1974. MR0375281 (51:11477)

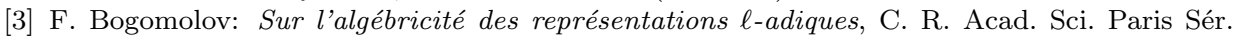
A-B 290 (1980), A701-A703. MR.574307(81c:14025)

[4] P. Deligne: Cohomologie Étale, Séminaire de Géométrie Algébrique du Bois-Marie, SGA $4 \frac{1}{2}$, avec la collaboration de J.-F. Boutot, A. Grothendieck, L. Illusie, et J.-L. Verdier. Lecture Notes in Mathematics 569, Springer-Verlag, 1977. MR0463174 (57:3132)

[5] P. Deligne, P. Griffiths, J. Morgan, D. Sullivan: Real homotopy theory of Kähler manifolds, Invent. Math. 29 (1975), 245-274. MR0382702 (52:3584)

[6] C. Earle, I. Kra: On sections of some holomorphic families of closed Riemann surfaces, Acta Math. 137 (1976), 49-79. MR0425183 (54:13140)

[7] J. Ellenberg: 2-nilpotent quotients of fundamental groups of curves, unpublished manuscript.

[8] W. Fulton, J. Harris: Representation theory. A first course. Graduate Texts in Mathematics, 129, Springer-Verlag, 1991. MR.1153249 (93a:20069)

[9] B. van Geemen, F. Oort: A compactification of a fine moduli space of curves, in Resolution of singularities (Obergurgl, 1997), 285-298, Progr. Math., 181, Birkhäuser, 2000. MR.1748624 (2001f:14049)

[10] M. Goresky, R. MacPherson: Stratified Morse Theory, Ergebnisse der Mathematik und ihrer Grenzgebiete 14, Springer-Verlag, 1988. MR932724 (90d:57039)

[11] A. Grothendieck: Éléments de géométrie algébrique. II. Étude globale élémentaire de quelques classes de morphismes, Inst. Hautes Études Sci. Publ. Math. No. 8, 1961. MR0217084 $(36: 177 \mathrm{~b})$

[12] A. Grothendieck: Letter to Faltings dated June 27, 1983. Available at http:// people.math.jussieu.fr/ leila/grothendieckcircle/letters.php.

[13] R. Hain: Completions of mapping class groups and the cycle $C-C^{-}$, Mapping class groups and moduli spaces of Riemann surfaces, 75-105, Contemp. Math., 150, Amer. Math. Soc. MR.1234261 (95e:14018)

[14] R. Hain: Torelli groups and Geometry of Moduli Spaces of Curves, Current topics in complex algebraic geometry (Berkeley, CA, 1992/93), 97-143, Math. Sci. Res. Inst. Publ., 28, Cambridge Univ. Press, 1995. MR1397061 (97d:14036)

[15] R. Hain: Infinitesimal presentations of Torelli groups, J. Amer. Math. Soc. 10 (1997), 597651. MR:1431828 (97k:14024)

[16] R. Hain: Relative weight filtrations on completions of mapping class groups, in Groups of Diffeomorphisms, Advanced Studies in Pure Mathematics, vol. 52 (2008), pp. 309-368, Mathematical Society of Japan. MR 2509715 (2010j:57001) 
[17] R. Hain: Lectures on Moduli Spaces of Elliptic Curves, in Transformation Groups and Moduli Spaces of Curves, Advanced Lectures in Mathematics, edited by Lizhen Ji, Shing-Tung Yau, no. 16 (2010), pp. 95-166, Higher Education Press, Beijing. arXiv:0812.1803.

[18] R. Hain: Monodromy of codimension-one sub-families of universal curves, arXiv:1006.3785.

[19] R. Hain: Remarks on non-abelian cohomology of proalgebraic groups, preprint, 2010.

[20] R. Hain, M. Matsumoto: Weighted completion of Galois groups and Galois actions on the fundamental group of $\mathbb{P}^{1}-\{0,1, \infty\}$, Compositio Math. 139 (2003), 119-167. MR2025807 (2005c:14031)

[21] R. Hain, M. Matsumoto: Tannakian fundamental groups associated to Galois groups, Galois groups and fundamental groups, 183-216, Math. Sci. Res. Inst. Publ., 41, Cambridge Univ. Press, Cambridge, 2003. MR2012217 (2004k:14036)

[22] R. Hain, M. Matsumoto: Galois actions on fundamental groups of curves and the cycle $C-C^{-}$, J. Inst. Math. Jussieu 4 (2005), 363-403. MR2197063(2007a:14027)

[23] R. Hain, M. Matsumoto: Weighted completion of arithmetic mapping class groups, in preparation.

[24] R. Hain, M. Matsumoto, G. Pearlstein, T. Terasoma: Tannakian fundamental groups of categories of variations of mixed Hodge structure, in preparation.

[25] J. Hubbard: Sur la non-existence de sections analytiques à la courbe universelle de Teichmüller, C. R. Acad. Sci. Paris Sér. A-B 274 (1972), A978-A979. MR0294719 (45:3787)

[26] D. Johnson: The structure of the Torelli group, III: The abelianization of I , Topology 24 (1985), 127-144. MR793179 (87a:57016)

[27] A. Kabanov: Stability of Schur functors, J. Algebra 195 (1997), 233-240. MR1468891 (99c:17014)

[28] M. Kim: The motivic fundamental group of $\mathbb{P}^{1}-\{0,1, \infty\}$ and the theorem of Siegel, Invent. Math. 161 (2005), 629-656. MR.2181717(2006k:11119)

[29] M. Kim: The unipotent Albanese map and Selmer varieties for curves, Publ. Res. Inst. Math. Sci. 45 (2009), 89-133. MR2512779 (2010k:14029)

[30] T. Kohno, T. Oda: The lower central series of the pure braid group of an algebraic curve, in Galois Representations and Arithmetic Algebraic Geometry, editor: Y. Ihara. Advanced Studies in Pure Mathematics 12 (1987), 201-219. MR948244(90a:14026)

[31] F. Knudsen: The projectivity of the moduli space of stable curves, III. The line bundles on $M_{g, n}$, and a proof of the projectivity of $\bar{M}_{g, n}$ in characteristic 0. Math. Scand. 52 (1983), 200-212. MR702954 (85d:14038b)

[32] J. Labute: On the descending central series of groups with a single defining relation, J. Algebra 14 (1970), 16-23. MR0251111 (40:4342)

[33] D. Mumford, J. Fogarty, F. Kirwan: Geometric invariant theory. Third edition. Ergebnisse der Mathematik und ihrer Grenzgebiete 34, Springer-Verlag, 1994. MR.1304906 (95m:14012)

[34] H. Nakamura, N. Takao, R. Ueno: Some stability properties of Teichmüller modular function fields with pro-l weight structures, Math. Ann. 302 (1995), 197-213. MR1336334 (96h:14041)

[35] J. Neukirch, A. Schmidt, K. Wingberg: Cohomology of number fields. Second edition. Grundlehren der Mathematischen Wissenschaften, 323, Springer-Verlag, 2008. MR2392026 (2008m:11223)

[36] B. Noohi: Fundamental groups of algebraic stacks, J. Inst. Math. Jussieu 3 (2004), 69-103. MR2036598 (2004k:14003)

[37] T. Oda: Etale homotopy type of the moduli spaces of algebraic curves, Geometric Galois actions, 1, 85-95, London Math. Soc. Lecture Note Ser., 242, Cambridge Univ. Press, Cambridge, 1997. MR1483111(2000a:14030)

[38] A. Putman: The second rational homology group of the moduli space of curves with level structures, preprint 2008, arXiv:0809.4477.

[39] W. Schmid: Variation of Hodge structure: The singularities of the period mapping, Invent. Math. 22 (1973), 211-319. MR0382272 (52:3157)

[40] J. Stallings: Homology and central series of groups, J. Algebra 2 (1965), 170-181. MR0175956 $(31: 232)$

[41] S. Zucker: Variation of mixed Hodge structure. II, Invent. Math. 80 (1985), 543-565. MR791674 (87h:32050b)

[42] J. Stix: A monodromy criterion for extending curves, Int. Math. Res. Not. 2005, 1787-1802. MR 2172341 (2006g:14048) 
[43] D. Sullivan: On the intersection ring of compact three manifolds, Topology 14 (1975), 275277. MR0383415 (52:4296)

[44] A. Weil: L'arithmétique sur les courbes algébriques, Acta Math. 52 (1929), 281-315. MR.1555278

[45] K. Wickelgren: Lower central series obstructions to homotopy sections of curves over number fields, Ph.D. thesis, Stanford University, 2009. MR.2713908

Department of Mathematics, Duke University, Durham, North Carolina 27708-0320

E-mail address: hain@math.duke.edu 